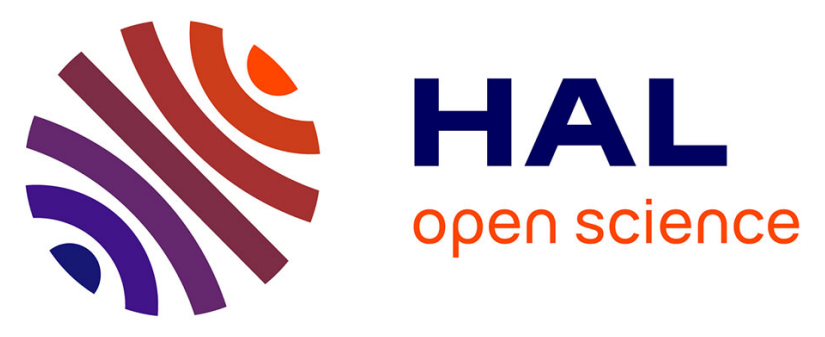

\title{
Nicotine inhibits the VTA-to-amygdala dopamine pathway to promote anxiety
}

Claire Nguyen, Sarah Mondoloni, Tinaïg Le Borgne, Ines Centeno, Maxime

Come, Joachim Jehl, Clément Solié, Lauren M. Reynolds, Romain Durand-de

Cuttoli, Stefania Tolu, et al.

\section{To cite this version:}

Claire Nguyen, Sarah Mondoloni, Tinaïg Le Borgne, Ines Centeno, Maxime Come, et al.. Nicotine inhibits the VTA-to-amygdala dopamine pathway to promote anxiety. Neuron, 2021, 109 (16), pp.2604-2615.e9. 10.1016/j.neuron.2021.06.013 . pasteur-03300623

\section{HAL Id: pasteur-03300623}

https://hal-pasteur.archives-ouvertes.fr/pasteur-03300623

Submitted on 24 Nov 2021

HAL is a multi-disciplinary open access archive for the deposit and dissemination of scientific research documents, whether they are published or not. The documents may come from teaching and research institutions in France or abroad, or from public or private research centers.
L'archive ouverte pluridisciplinaire HAL, est destinée au dépôt et à la diffusion de documents scientifiques de niveau recherche, publiés ou non, émanant des établissements d'enseignement et de recherche français ou étrangers, des laboratoires publics ou privés.

\section{(ㅇ)(1) $\$$}

Distributed under a Creative Commons Attribution - NonCommercial - NoDerivatives $\mid 4.0$ 


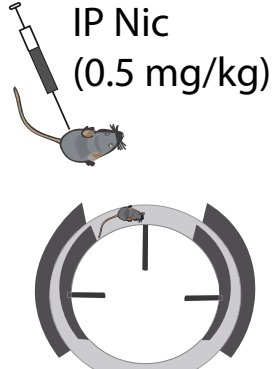

Nicotine-evoked anxiety

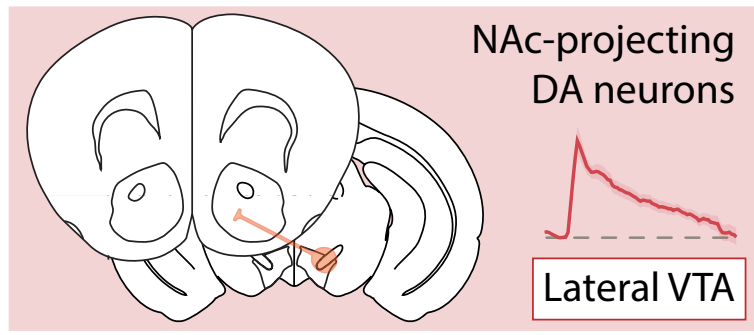

NAc DA terminal activation or inhibition $((-7))$ No change in anxiety

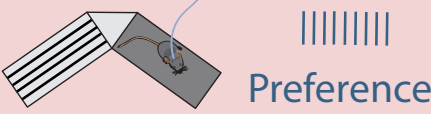
nicotine-evoked anxiety
Abstract;Graph abstract.pdf

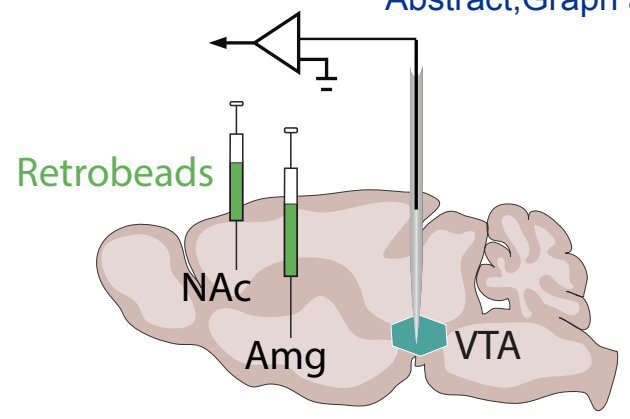

DA fring activation

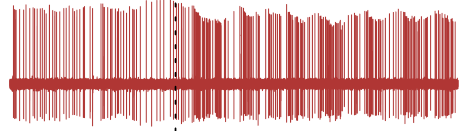

Nicotine

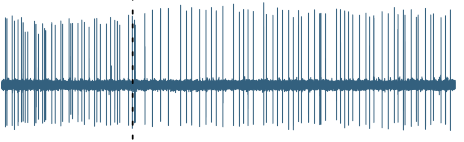

DA firing inhibition

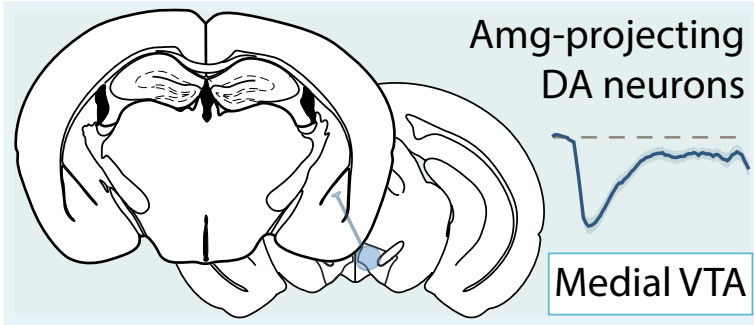

Amg DA terminal activation or inhibition
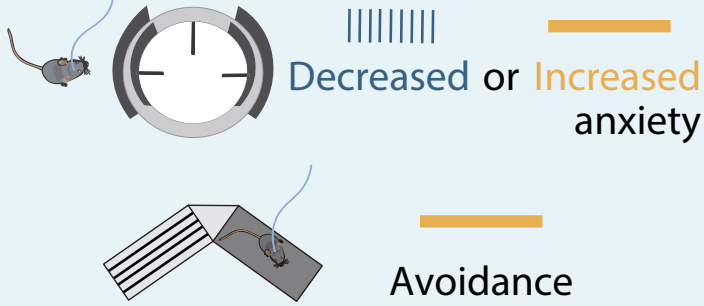

Avoidance

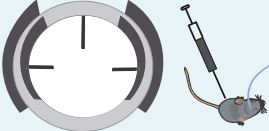

||||||||| Suppresses nicotine-evoked anxiety 


\section{Nicotine inhibits the VTA to Amygdala dopamine pathway to promote 2 anxiety}

\section{Authors}

4 Nguyen $\mathrm{C}^{1,2}$, Mondoloni S2, Le Borgne $\mathrm{T}^{1,2}$, Centeno $\mathrm{I}^{2}$, Come $\mathrm{M}^{1,2}$, Jehl $\mathrm{J1}^{1,2}$, Solié $\mathrm{C}^{1,2}$, Reynolds LM ${ }^{1,2}$, 5 Durand-de Cuttoli $\mathrm{R}^{2}$, Tolu $\mathrm{S}^{2}$, Valverde $\mathrm{S}^{2}$, Didienne $\mathrm{S}^{1,2}$, Hannesse $\mathrm{B}^{2}$, Fiancette $\mathrm{JF}^{3}$, Pons $\mathrm{S}^{4}$, Maskos

$6 \mathrm{U}^{4}$, Deroche-Gamonet $\mathrm{V}^{3}$, Dalkara $\mathrm{D}^{5}$, Hardelin $\mathrm{JP}^{1,2}$, Mourot $\mathrm{A}^{1,2}$, Marti $\mathrm{F}^{1,2 \#^{*}}$ \& Faure $\mathrm{P}^{1,2 \#^{*}}$

\section{Affiliations}

1 ESPCI, Laboratoire de plasticité du cerveau UMR8249, 10 rue Vauquelin, 75005 Paris, France.

2 Sorbonne Université, Inserm, UMR8246 CNRS, Neuroscience Paris Seine - IBPS, 75005 Paris, France

${ }^{3}$ Neurocentre Magendie, Inserm U1215, Université de Bordeaux, 146 rue Léo Saignat, Bordeaux F33077, France

${ }^{4}$ Institut Pasteur, Unité Neurobiologie intégrative des systèmes cholinergiques, Département de neuroscience, 75724 Paris cedex, France.

${ }^{5}$ Sorbonne Université, Inserm, CNRS, Institut de la Vision, Paris, France.

\# equal contributions

*Correspondence to fabio.marti@upmc.fr, phfaure@gmail.com

Lead contact: phfaure@gmail.com

\section{Summary}

Nicotine stimulates dopamine (DA) neurons of the ventral tegmental area (VTA) to establish and maintain reinforcement. Nicotine also induces anxiety, through an as yet unknown circuitry. We found that nicotine injection drives opposite functional responses of two distinct populations of VTA DA neurons with anatomically segregated projections: it activates neurons that project to the nucleus accumbens (NAc), whereas it inhibits neurons that project to the amygdala nuclei (Amg). We further show that nicotine mediates anxiety-like behavior by acting on $\beta 2$ subunit-containing nicotinic acetylcholine receptors of the VTA. Finally, using optogenetics, we bidirectionally manipulate the VTA-NAC and VTA-Amg pathways to dissociate their contributions to anxiety-like behavior. We show that inhibition of VTA-Amg DA neurons mediates anxiety-like behavior, while their activation prevents the anxiogenic effects of nicotine. These distinct subpopulations of VTA DA neurons with opposite responses to nicotine may differentially drive the anxiogenic and the reinforcing effects of nicotine.

\section{Keywords}

nicotinic acetylcholine receptors; dopamine circuits; addiction; juxtacellular recordings; optogenetics; amygdala; nucleus accumbens; ventral tegmental area 
Nicotine is the principal addictive component that drives continued tobacco use. The initiation of addiction involves the mesocorticolimbic dopamine (DA) system, which contributes to the processing of rewarding stimuli during the overall shaping of successful behaviors (Schultz, 2007). Addictive drugs such as nicotine are assumed to hijack the mechanisms of reinforcement learning, leading to an overvaluation of the drug reward at the expense of natural rewards. While drug-induced reinforcement learning generally involves an increase in extracellular DA concentration in the nucleus accumbens (NAc), the underlying molecular and cellular mechanisms are drug dependent (Changeux, 2010; Di Chiara and Imperato, 1988; Luscher, 2016). Nicotine exerts its reinforcing effects through the direct activation of nicotinic acetylcholine receptors (nAChR), a family of pentameric ligand-gated ion channels (Changeux et al., 1998), expressed on midbrain DA and GABA neurons, thus increasing the activity of both neuronal populations (Maskos et al., 2005; Morel et al., 2014; Tolu et al., 2013). Cell type-specific optogenetic manipulations have confirmed that DA cell activation is sufficient to drive the transition toward addiction, and established causal links between DA neuron activation and drug-adaptive behaviors (Pascoli et al., 2015). However, such a view does not take into account the heterogeneity of midbrain DA neurons and the possibility that different messages can be transmitted in parallel from DA neurons of the ventral tegmental area (VTA DA neurons). Indeed, VTA DA neurons belong to anatomically distinct circuits, differ in their molecular features, and show diverse responses to external stimuli (Lammel et al., 2008; Poulin et al., 2018). DA neurons transmit signals related not only to salience and reward, but also to aversive stimuli (Brischoux et al., 2009; de Jong et al., 2019), including the "negative" effects of nicotine at high doses (Grieder et al., 2019; 2010). However, how DA neurons simultaneously drive opposite outcomes in response to the same stimuli remains unclear. While the vast majority of research groups that have examined nicotine-evoked responses report a homogenous activation of $\mathrm{DA}$ neurons and an increase in $\mathrm{DA}$ release in their projection areas (Di Chiara and Imperato, 1988; Grenhoff et al., 1986; Mansvelder and McGehee, 2000; Maskos et al., 2005; Picciotto et al., 1998; Zhao-Shea et al., 2011), other reports suggest that the responses of VTA DA neurons to nicotine are more heterogeneous than previously thought (Eddine et al., 2015; MameliEngvall et al., 2006; Zhao-Shea et al., 2011). Therefore, a key issue is how the multiple effects of nicotine map onto DA cell diversity, and whether nAChR or other features can define different neuronal subpopulations that, through their response to nicotine, can influence specific behaviors.

\section{Results}

\section{Distinct VTA DA neuron populations show opposite responses to acute nicotine injection}

We recorded the response of VTA DA neurons to an intravenous (IV) injection of nicotine using singlecell electrophysiological recordings in anesthetized mice. We used a dose of nicotine $(30 \mu \mathrm{g} / \mathrm{kg})$ that has been shown to be reinforcing in the context of IV self-administration (Morel et al., 2014). These neurons were first identified during the recordings based on their electrophysiological properties (i.e., firing rate and action potential width) (Mameli-Engvall et al., 2006; Ungless and Grace, 2012), and then filled with neurobiotin (NB) by the juxtacellular labeling technique (Eddine et al., 2015; Pinault, 1996). All neurons were confirmed as DA neurons by post hoc immunofluorescence with co-labeling for tyrosine hydroxylase $(\mathrm{TH})$ and NB (Figure $1 \mathrm{~A})$. Acute IV nicotine injections induced a significant variation of DA neuron firing rates, producing either an increase or a decrease in firing rate that was absent in control experiments with 
saline. Indeed, the variations in firing frequency had a unimodal distribution for saline injections $(n=233$ neurons) but a bimodal distribution for nicotine injections ( $n=245$, Figure $1 B$, comparison of distribution, Kolmogorov-Smirnov test, $p<0.001$, see also Figure S1). Among the 245 identified DA neurons, some were activated ( $\mathrm{Nic}+, n=155)$ whereas others were inhibited Nic,$- n=88$ ) by the nicotine injection (Figure 1C), in line with our previous findings (Eddine et al., 2015). Nicotine-induced increases or decreases in DA neuron firing rate were of similar amplitude (about 35\% from baseline for a dose of 30 $\mu \mathrm{g} / \mathrm{kg}$ ), and were higher in amplitude than saline-evoked responses (Figure 1D). In addition, nicotineinduced changes in DA neuron firing rate were dose-dependent and, importantly, maintained the polarity of their response (i.e. either an increase or decrease) at all doses tested (Figure 1E). Finally, to rule out potential confounding effects of anesthesia on the activity of VTA DA neurons, putative VTA DA neurons $(n=16)$ were recorded in freely-moving mice (Figure S2A-B), and nicotine or saline was injected into the tail vein (IV $30 \mu \mathrm{g} / \mathrm{kg}$, see methods). We observed VTA DA neurons that were either activated ( $\mathrm{Nic}+, \mathrm{n}$ $=8$ ) or inhibited (Nic -, $n=8$ ) by the nicotine injection (Figure $1 F$ ), replicating the results we found in anesthetized mice (comparisons between saline-induced and nicotine-induced firing rate variations by Student's t-test with Bonferroni correction, ${ }^{*} p=0.02$ for activated neurons and ${ }^{* * *} p<0.001$ for inhibited neurons). Therefore, the nature of nicotine-evoked responses (i.e. activation or inhibition) constitutes a marker that allows the robust segregation of VTA DA neurons into two populations.

We then sought to determine whether the spontaneous activity of these two populations of DA neurons differ in anesthetized mice. The basal activity of VTA DA neurons is characterized by the firing rate and the percentage of spikes within a burst (\% SWB) (Mameli-Engvall et al., 2006). Bursts are classically identified as discrete events consisting of a sequence of spikes with (1) a burst onset defined by two consecutive spikes within an interval $<80 \mathrm{~ms}$ and (2) the end of a burst defined by an inter-spike interval $>160 \mathrm{~ms}$ (Grace and Bunney, 1984a; Ungless and Grace, 2012). We found that nicotine-activated and nicotine-inhibited DA neurons had similar firing rates $(\Delta=0.26 \mathrm{~Hz}, p=0.0506)$ and bursting activities $(\Delta$ $=3.5 \%, p=0.064$ Figure $S 2 C$ ). An analysis of the distribution of burst time intervals also highlighted different profiles in the distribution of inter-spike intervals depending on the burst length (Figure S2C). Other parameters describing cell spontaneous activity (e.g. coefficient of variation or bursting frequency) were analyzed, but none of them revealed a difference between nicotine-activated and nicotine-inhibited DA neurons. Finally, a multiple logistic regression was used to predict the probability of response type (inhibited/activated) based on predictor variables (the firing frequency, the coefficient of variation, \%SWB and bursting frequency). Only the spontaneous firing frequency was statistically associated to the outcome $(p=0.007$ ) and the classification prediction was very low (about $36 \%$ ). Overall, differences between the two groups could be detected, yet nicotine-evoked responses could not be predicted based upon the sole analysis of spontaneous activity.

We next asked whether these two populations were anatomically segregated. Neurobiotin-filled cell bodies of each responding neuron $(n=243)$ were positioned onto mouse brain atlas plates (Paxinos and Franklin, 2004) (Figure S3) to study their anatomical location. As illustrated by a single atlas plate schematic (bregma - $3.3 \mathrm{~mm}$ ), anatomical coordinates suggest that the inhibited neurons were located more medially within the VTA than the activated neurons, independently of their antero-posterior or dorsoventral positions (Figure 1G).

Nicotine-activated VTA DA neurons project to the nucleus accumbens, while nicotine-inhibited VTA DA neurons project to the amygdala 
The DA system is heterogeneous, and is increasingly thought about in terms of anatomically and functionally distinct sub-networks (Watabe-Uchida et al., 2012). DA neurons in the VTA have been reported to project to different terminal regions based on their localization along the mediolateral axis (Beier et al., 2019; 2015; Lammel et al., 2008). Therefore, we next investigated whether these two subpopulations belong to anatomically distinct dopamine circuits by probing nicotine-evoked responses of DA neurons with identified projection sites. To do so, we first targeted the nucleus accumbens (NAc) by simultaneously injecting green retrobeads (RB), a retrograde tracer, in 3 sub-nuclei: the lateral shell (NAcLSh), the medial shell (NAcMSh) and core (Figure S4A). Two weeks later, spontaneous and nicotineevoked activities of VTA DA neurons were recorded in vivo in anesthetized mice, and neurons were then labeled with neurobiotin. Triple labeling immunofluorescence allowed us to confirm post hoc the DA nature $(\mathrm{TH}+)$, projection site (RB+ / $\mathrm{RB}-)$, and position $(\mathrm{NB}+)$ of all recorded neurons (Figure 2A, Figure S4B). We recorded and labeled 32 nicotine-activated and 17 nicotine-inhibited neurons in mice with RB injected in the NAc (all shell+core), among which 30 neurons were further identified as NAc-projecting $(\mathrm{RB}+, \mathrm{TH}+)$ neurons. Out of the NAc-projecting DA neurons 93\% (28/30) were activated by nicotine, while only $7 \%$ (2/30) of neurons were inhibited. In contrast, the remaining 19 DA neurons showed no evidence of projection to the NAc (RB-, $\mathrm{TH}+$ ), and $79 \%$ (15/19) of these neurons were inhibited by a nicotine injection, while $21 \%(4 / 19)$ were activated (Figure 2B-C). The proportion of nicotine-activated neurons in NAcprojecting cells was thus significantly greater than what would be expected from the entire population of $\mathrm{RB}+$ and RB- neurons (Pearson's Chi-squared test, $\mathrm{p}<0.001$ ). A similar analysis was carried out on mice with a single RB injection site of either the NAcMSh or the NAcLSh to examine whether this effect was driven by a specific NAc sub-nucleus. Analysis of the nicotine-evoked responses on NAcMSh-projecting DA neurons $(n=14 \mathrm{RB}+, \mathrm{TH}+$ and $n=8 \mathrm{RB}-\mathrm{TH}+)$ and NAcLSh-projecting DA neurons ( $\mathrm{n}=6 \mathrm{RB}+, \mathrm{TH}+$ and $n=6 \mathrm{RB}-\mathrm{TH}+$ ) leads to the same conclusion that the majority of DA neurons that project to the NAc are activated by nicotine, regardless of the specific NAc sub-nucleus they project to (Figure S5). In a second series of experiments, RB were injected in the amygdala nuclei (Amg), targeting both the basolateral $(B L A)$ and central $(\mathrm{CeA})$ amygdala (Figure $\mathrm{S} 4 \mathrm{C})$. All recorded neurons were once again labeled with NB and confirmed as DA post hoc by triple labeling immunofluorescence $(\mathrm{TH}+, \mathrm{NB}+, \mathrm{RB}+/-$, Figure 2D, Figure S4D). We recorded and labeled 26 nicotine-activated and 26 nicotine-inhibited neurons in mice with RB injected in the Amg (BLA + CeA) (Figure 2E-F), among which 22 VTA DA neurons were confirmed as Amg-projecting (RB+, TH+) neurons. Out of the Amg-projecting DA neurons, 86\% (19/22) were nicotine-inhibited, while only $14 \%(3 / 22)$ were activated. In contrast, DA neurons without evidence of projection to the Amg (RB-, $\mathrm{TH}+$ ) were mainly nicotine-activated $(77 \%, 23 / 30)$, with $23 \%(7 / 30)$ of neurons inhibited (Figure 2E-F). The proportion of inhibited neurons in Amg-projecting cells was thus significantly greater than what would be expected from the entire population of RB+ and RB- neurons (Pearson's Chi-squared test, $p<0.001$ ). Analysis of the distribution of the nicotine-evoked variation in firing frequency for NAc-projecting $(n=30)$ and Amg-projecting neurons $(n=22)$ revealed two different distributions (Kolmogorov-Smirnov test, $p<0.001$ ) with opposite modes (i.e positive and negative variations, Figure $2 \mathrm{G}$ ). Overall, these results indicate that the majority of VTA DA neurons activated by an IV nicotine injection project to the NAc (core or shell), whereas the majority of nicotine-inhibited neurons project to the Amg (comparison of the percentages of inhibited and activated neurons in Amgprojecting neurons and NAc-projecting neurons by Pearson's Chi-squared test: $p<0.001$ ). Notably, in line with previous reports (Lammel et al., 2008), further anatomical analysis of triple-labeled VTA sections revealed that Amg-projecting DA neurons are located more medially in the VTA than NAc-projecting DA neurons (Figure S4E). We found that NAc-projecting and Amg-projecting DA neurons had similar firing 
rates $(p=0.8)$ but Amg-projecting neurons tend to have higher bursting activity $(p=0.28)$, in line with what we had previously observed for nicotine-activated and nicotine-inhibited VTA DA neurons (Figure $\mathrm{S} 4 \mathrm{~F}$, and see Figure $\mathrm{S} 2 \mathrm{C}$ ). We then probed how these opposite changes in the firing of VTA DA neurons in response to nicotine injection translate into dopamine release in the NAc and Amg. Using in vivo fiber photometry and a genetically-encoded dopamine sensor (GRABDA2m, (Sun et al., 2018; 2020)) we assessed the real-time dynamics of DA release in the NAcLSh and in the BLA after IV nicotine injection $(30 \mu \mathrm{g} / \mathrm{kg})$ in the tail vein of freely-moving mice (Figure $2 \mathrm{H}$ ). We found that nicotine injection evoked an increase of DA release in the NAcLSh, whereas it produced a decrease of DA release in the BLA (Figure 21), in agreement with our electrophysiological results at the cell body level. Together, these results confirm that nicotine drives opposite functional responses within two distinct DA pathways from the VTA.

Finally, we took advantage of the anatomical distinction between these two pathways to analyze the respective electrophysiological properties of their VTA DA neurons in ex vivo patch-clamp recordings. NAc-projecting (MSh+LSh+core) or Amg-projecting (BLA+CeA) DA neurons were labeled with RB (Figure S6A-B). Amg-projecting DA neurons showed higher excitability (Figure S6C-D) than NAc-projecting DA neurons, but no difference in nicotine-evoked currents was found between these two populations (Figure S6E-F). These results indicate that these two VTA DA cell populations have different membrane properties, but do not markedly differ in the functional expression of somatodendritic nAChR.

\section{The anxiogenic effect of nicotine requires $\beta 2$ subunit-containing $n A C h R$ in the VTA}

178

179

180

181

182

183

184

185

186

187

188

We next asked whether these two distinct dopamine sub-circuits are associated with different behavioral outcomes after an acute injection of nicotine. Nicotine is known to have rewarding properties, which require the activation of VTA DA neurons (Durand-de Cuttoli et al., 2018; Maskos et al., 2005; Tolu et al., 2013). However, nicotine can also induce negative outcomes such as anxiety-like behaviors and stressinduced depressive-like states (Kutlu and Gould, 2015; Morel et al., 2017; Picciotto and Mineur, 2013), for which the underlying circuitry remains elusive. We hypothesized that the activation and inhibition of the different DA neuron pathways have distinct roles in nicotine-induced behavior. We first aimed to establish the role of the VTA in the anxiogenic effects of acute nicotine exposure. To this end, mice were placed in an elevated-O-maze (EOM) after an acute injection of either saline or nicotine (intra-peritoneal, $\mathrm{IP}, 0.5 \mathrm{mg} / \mathrm{kg}$, injected one minute before the test), and we found that nicotine, but not saline, reduced exploration of the open arms over time (Figure 3A and Figure S7A for individual data). Mice that received nicotine injections also showed fewer entries into the open arms (Figures S7A), and this anxiety-like phenotype was not related to a detectable effect of nicotine on locomotor activity in an open field (OF, Figure S7B). Next, to probe the specific role of the VTA in this anxiogenic effect, we locally infused nicotine into this brain region (Figure S7C) one minute before the EOM test, using bilaterally implanted cannulas. As with IP injections, we found that the infusion of nicotine, but not saline, directly into the VTA decreased exploration of the EOM open arms over time (Figure 3B and Figure S7D for individual data). Finally, we assessed the involvement of VTA $\beta 2$ subunit-containing $n A C h R\left(~\left(\beta 2^{*} \mathrm{nAChR}\right)\right.$ in the anxiogenic effect of nicotine, as nicotine-evoked responses have been shown to be mainly mediated by $\beta 2^{*} \mathrm{nAChR}$ present on the soma of both DA and VTA GABA neurons (Tolu et al., 2013). In vivo juxtacellular recordings of VTA DA neurons in mutant mice lacking the $\beta 2$ subunit of $n A C h R$ ( $\beta 2--$ mice) demonstrated the absence of a response to nicotine injection (Figure $3 \mathrm{C}$ left). Lentiviral re-expression of the $\beta 2$ subunit selectively in the VTA of $\beta 2--$ mice ( $\beta 2--\mathrm{Vec}$ mice) globally restored the response to nicotine injection (Figure $3 \mathrm{C}$ and 
Figure S8), allowing the reemergence of nicotine-induced increases or decreases in DA neuron firing 202 (Figure $3 \mathrm{C}$ left). Regarding behavior, $\beta 2-$ mice were insensitive to the anxiogenic effect of nicotine injection in the EOM test and lentiviral re-expression of $\beta 2$ in the VTA ( $\beta 2--V e c$ mice) restored this effect (Figure 3D and see Figure S7G for individual data). Together, these results indicate that the anxiogenic effect of an acute nicotine injection requires signaling through $\beta 2^{*} n A C h R$ in the VTA, but do not allow us to conclude whether the activation and/or inhibition of specific VTA DA neuron populations is required.

\section{Manipulating the VTA-Amg DA pathway modulates basal and nicotine-induced anxiety}

208

209

210

211

212

213

214

215

216

217

218

219

220

221

222

223

224

225

226

227

228

229

230

231

232

233

234

235

236

237

238

239

240

241

Ideally, dissociating whether nicotine-evoked activation or inhibition of VTA DA neurons is necessary for the behavioral effects of nicotine would require to isolate these responses in DA neurons, as well as in VTA GABA neurons, which also express nAChR (Grieder et al., 2019; Tolu et al., 2013). However, because nicotine-induced activation and inhibition of DA neurons are concomitant and inextricably linked to one another (since nicotine cannot directly inhibit neurons), and because the responses of VTA DA and GABA neurons to nicotine are also tightly linked (Tolu et al., 2013), we decided to manipulate the two populations of DA neurons independently, using optogenetics. DAT-Cre mice expressing CatCh, Jaws (Figure S9) or YFP with no opsin (Figure S10A-B) were implanted in the BLA (Figure S10C) or in the NAcLSh (Figure S10D) to restrict the effects of the optogenetic stimulation to DA terminals within that region.

We first examined the effect of optogenetic manipulations of DA terminals in the amygdala. When compared to YFP controls, photo-inhibiting DA neuron terminals in the BLA of Jaws-expressing mice reduced the percentage of time spent in the open arms of the EOM, (Figure 4A, and see Figure S11A for individual data). There were also no detectable effects of the light-stimulation on the number of entries in the open arms (Figure S11A-B) or on locomotor activity (Figure S11C). Conversely, photo-activating DA terminals in the BLA of CatCh-expressing mice increased the percentage of time spent in the open arms of the EOM in comparison to mice expressing YFP (Figure 4B, and see Figure S11B for individual data). Moreover, we also noticed that the position of the animal at the onset of the stimulation did not impact any of the behavior observed in the EOM (Figure S11A-B). To determine whether the anxiogenic effect observed during inhibition of DA neuron terminals in the BLA was specific to the BLA nucleus, we used another group of WT mice injected with either Jaws or GFP in the VTA, and implanted bilateral optical fibers either in the BLA or in the CeA (Figure S12A-B). We found that optogenetically inhibiting VTA neuron terminals in Jaws-expressing WT mice decreased the percentage of time spent in the open arms of the EOM when optical fibers were implanted in the BLA, but not when they were implanted in the CeA (Figure S12C-D). There was not a detectable effect of stimulation on locomotor activity in an OF (Figure S12E-F). We next asked whether optogenetically activating the terminals of BLA-projecting VTA DA neurons could prevent the anxiogenic effect of nicotine injection. DAT-Cre mice expressing CatCh or YFP only in the VTA received an IP injection of nicotine one minute before the EOM test, and received light stimulation in the BLA throughout the 9-minute test. Indeed, we found that the light-evoked activation of BLA terminals of DA neurons during the EOM test abolished the anxiogenic effect of the nicotine injection, as the percentage of time spent by CatCh-expressing mice in the EOM open arms did not decrease during the test, and was significantly higher in these mice than in YFP-expressing controls during the last 3minute period of the test (Figure $4 \mathrm{C}$ ). We next explored the behavioral outcome of manipulating the terminals of BLA-projecting VTA DA neurons on motivational valence by using a real-time place 
preference paradigm (RTPP). Photo-inhibiting DA terminals in the BLA resulted in a significant avoidance for the compartment where animals were photo-stimulated, in keeping with our previous findings that inhibition of this pathway produces an anxiogenic effect in the EOM test, while photo-activating these terminals had no behavioral effect (Figure 4D). Inhibition of BLA-projecting VTA DA neurons therefore plays a central role in mediating the anxiogenic effect of nicotine.

\section{The VTA-NAc DA pathway is not involved in nicotine-induced anxiety-like behavior}

DA in the NAc has been suggested to be involved in the modulation of anxiety-like behavior (Radke and Gewirtz, 2012; Zarrindast et al., 2012). We thus next assessed whether NAc-projecting neurons also participate in the anxiogenic effects of acute nicotine administration. We examined the behavioral outcome of optogenetic manipulations of DA neuron terminals in the NAC during the EOM test. Lightevoked activation (CatCh-expressing mice, Figure 5A) or inhibition (Jaws-expressing mice, Figure 5B) of DA neuron terminals in the NAcLSh had no effect on the time spent in the open arms of the EOM (see Figure S13A-B for individual data). There was also no detectable effect of the light-stimulation on the number of entries in the open arms (Figure S13A-B), or on locomotor activity (Figure S13C). Moreover, the position of the animal at the onset of the stimulation did not reveal any impact on the behavior observed in the EOM (Figure S13A-B). Selectively inhibiting NAcLSh DA terminals using Jaws produced a slight change in basal anxiety levels but, more importantly, did not attenuate the anxiogenic effect of nicotine in the EOM test, as the drug reduced the exploration of the open arms over time in both control and opsin group (Figure 5C). NAcLSh-projecting VTA DA neurons are therefore not involved in mediating the anxiogenic effect of nicotine. In contrast, activation of DA neuron terminals in the NAcLSh induced significant place preference in the RTPP protocol, indicating that stimulating this pathway is rewarding (Figure 5D). Because medial and lateral NAc areas have different functional roles (de Jong et al., 2019), we further investigated the effect of optogenetic modulation of VTA neuron terminals in the NAcMSh in a separate group of WT mice (Figure S14A). Stimulating these terminals produced an increased number of entries and time spent in the EOM open arms, but this likely results from an increase of locomotor activity, as the distance traveled in an open field was likewise increased (Figure S14B-C). Inhibiting these terminals produced a slight decrease in basal anxiety levels but did not induce behavioral change in the EOM test nor prevented the reduction of time spent in open arms over time induced by nicotine (Figure S14B-D). Finally, we did not observe a significant effect of activating or inhibiting these terminals on the place preference score in the RTPP (Figure S14E). Our results thus demonstrate that NAcMSh and NAcLSh projections of VTA DA neurons are not involved in the nicotine-induced anxiety-like behavior observed in the EOM test.

\section{Discussion}

The VTA has long been perceived as a structure that broadly disseminates DA in the brain, with the different time courses of DA release providing a phenomenological account for the functional involvement of DA neurons in different behavioral processes (Schultz, 2007). This temporal account of DA neuron function was gradually replaced or extended by the notion that the DA system, in particular the VTA, is divided into subpopulations of DA neurons, each associated with distinct appetitive, aversive, or attentional behaviors (Lammel et al., 2012). However, we are only beginning to appreciate how the

281 functional activation/inhibition dynamics within these subpopulations impact behavioral processes. Here, 
we show that (1) activation and inhibition of VTA DA neurons appear concurrently as a consequence of nicotine injection, and (2) they correspond to two anatomically and functionally distinct circuits, which mediate contrasting behavioral effects. Our results argue for a functional dissociation of VTA to Amg and VTA to NAc DA pathways: inhibition of Amg-projecting VTA DA neurons is anxiogenic, while activation of NAcLSh-projecting VTA DA neurons is rewarding. We cannot completely rule out the possibility that optogenetic excitation of axon terminals produces backpropagation of action potentials and activation of other pathways. However, as the VTA projections to the NAc and Amg are anatomically segregated (i.e. neurons do not send collaterals to these two regions (Beier et al., 2015), it is unlikely that this would directly affect the functional dissociation between the two pathways studied in this paper. Furthermore, the fact that photoactivation of NAcLSh terminals is reinforcing, but not those of the NAcMSh or BLA, argues against this possibility.

VTA DA neurons are known to be heterogeneous in their axonal projections, electrophysiological properties, and in several molecular features. For example, they show striking differences in their expression of hyperpolarization-activated cyclic nucleotide-gated cation channels $(\mathrm{HCN})$, of the dopamine transporter (DAT), of the dopamine receptor D2R, and vesicular glutamate transporters (VGLUTs) (Lammel et al., 2008; Margolis et al., 2008; Morales and Margolis, 2017). However, the functional consequences of this heterogeneity on behavior remain poorly understood. Here, we demonstrate that nicotine injection evokes opposite responses in two distinct subpopulations of VTA DA neurons: a large majority of those with axons projecting to the NAc are activated, while a large majority of those with axons projecting to the Amg are inhibited. In addition to their functional and anatomical segregation, we found that these subpopulations display different excitabilities in vitro and different bursting activities in vivo. However, they cannot be distinguished solely on the basis of their spontaneous firing pattern in anesthetized mice. Are there specific intrinsic differences between these two neuronal populations, beside their projection sites, that would underlie their opposing responses to nicotine injection? NAcMShprojecting VTA DA neurons exhibit smaller $I_{h}$ currents than BLA-projecting VTA DA neurons, but both have similar input resistances and capacitances (Ford et al., 2006), and NAc core- and BLA-projecting neurons have similar expressions of DAT, D2R and TH (Su et al., 2019). We have previously reported that nicotine activated and inhibited VTA DA cells react similarly to D2R agonist or antagonist injection in vivo, in agreement with similar D2R expression levels in the two neuronal populations (Eddine et al., 2015). Finally, there is no clear variation in nicotine-evoked currents in Amg-projecting or NAc-projecting VTA neurons, suggesting that nAChR expression does not differ markedly between these populations.

While intrinsic differences may still exist, it is also possible that the emergence of either nicotine-evoked activation or inhibition of these neurons by nicotine arises from network dynamics. Nicotine's primary action is to activate $\mathrm{nAChR}$, which are well-characterized ligand gated cation channels, and cause neuronal depolarization. Within the VTA, nicotine directly activates both DA and GABA neurons, which both express nAChR (Klink et al., 2001; Tolu et al., 2013). In particular, $\beta 2^{*} n A C h R$ of the VTA neurons are key mediators of the reinforcement effects of nicotine, as previously shown by re-expressing the $\beta 2$ subunit of nAChR locally in the VTA of $\beta 2^{---}$mice (Maskos et al., 2005; Tolu et al., 2013), or by rendering $\beta 2^{*} n A C h R$ insensitive to nicotine using light (Durand-de Cuttoli et al., 2018). Here, we show that $\beta 2^{*} n A C h R$ of VTA neurons are also required to evoke, after systemic nicotine injection, the anxiogenic properties of nicotine as well as the inhibition of the subpopulation of DA neurons projecting to Amg. Therefore, nicotine acting through $\beta 2^{*} n A C h R$ activates VTA GABAergic interneurons and DA neurons 
projecting to the NAc, while concurrently inhibiting DA neurons projecting to the Amg. The inhibitory effect of nicotine may be mediated by inhibition through local DA release (Eddine et al., 2015), although no difference in D2R-mediated inhibitory postsynaptic currents or in DA reuptake between NAcMShprojecting and BLA-projecting DA neurons has been reported (Ford et al., 2006). Alternatively, it could involve either local (interneurons) or long-range GABAergic inhibition of the Amg-projecting DA neuron subpopulation primarily, which is compatible with the recent demonstration of distinct inhibitory networks resulting in specific feedback loops between VTA and NAc sub-regions (Yang et al., 2018).

Nicotine is highly reinforcing, but also produces aversive and anxiogenic effects at various doses (Balerio et al., 2006; Kutlu and Gould, 2015; Picciotto and Mineur, 2013; Wolfman et al., 2018). Importantly, as the doses of nicotine used in this study are known to be rewarding in different paradigms in mice, an effect attributable to VTA DA neuron activation (Durand-de Cuttoli et al., 2018; Maskos et al., 2005; Tolu et al., 2013), we demonstrate that the same dose of nicotine can concurrently induce a rewarding effect by activating the VTA to NAc DA pathway, and a "negative" emotional state by inhibiting the VTA to Amg DA pathway. Yet, we find that neither the activating effects nor the inhibiting effects of nicotine injection on VTA DA neurons can override each other, that is to say that both types of responses occur at each dose of nicotine along the dose-response curve, with neither response taking precedence at any specific dose. Thus, depending on the context, the exact same dose of nicotine can trigger anxiety or reinforcement. Aversion for high doses of nicotine and anxiety associated with nicotine withdrawal have been attributed to nicotinic and glutamatergic signaling in the habenulo-interpeduncular axis (Fowler et al., 2011; Frahm et al., 2011; Molas et al., 2017; Zhao-Shea et al., 2013). There is also evidence that nAChR of neurons located in the Amg modulate depressive-like states (Mineur et al., 2016). However, a role for DA in aversion to nicotine has also been proposed. D1R and D2R antagonists prevent conditioned-place aversion induced by an acute high-dose nicotine injection (Grieder et al., 2012), and $\beta 2^{*} n A C h R$ have been shown to be necessary for both the aversive and rewarding effects of nicotine by a strategy of $\beta 2$ subunit re-expression in DA and GABAergic neurons of the VTA in $\beta 2^{---}$mice (Grieder et al., 2019). However, the mechanism underlying these opposite effects of the drug has not yet been established. Here, we show that activation of $\beta 2^{*} n A C h R$ of VTA neurons is necessary for nicotine to inhibit Amgprojecting DA neurons and induce anxiety-like behavior. This indicates that VTA signaling is critically involved in the acute anxiogenic effect of nicotine, and suggests that it could also mediate aversion to nicotine. Our experiments also demonstrate that inhibition of the VTA to Amg DA pathway allows the expression of anxiety-like behavior, and that a reduction of this inhibition relieves nicotine-induced anxiety-like behavior. These experiments strongly suggest a driving role for the inhibition of this pathway in nicotine-induced anxiety behavior, yet they do not exclude the possibility that other pathways also transmit the anxiogenic effect of nicotine.

358 Our findings emphasize the complex role of the DA system in not only positive but also negative motivational processes, proposing a more nuanced view of the effects of reinforcing doses of nicotine on VTA DA neurons. Opposing responses of DA neurons to drug exposure have also been observed with cocaine (Mejias-Aponte et al., 2015), ethanol (Doyon et al., 2013), and morphine (Margolis et al., 2014).

362 Notably, the inhibition of VTA DA neurons induced by opioids differs according to their NAc or BLA 363 projection zone (Ford et al., 2006), suggesting that the behavioral effects of opioid drugs could also result 364 from a specific pattern of inhibition in these two pathways. Since our results demonstrate that both 365 
366 distinct subpopulations of VTA DA neurons, the question then arises as to how the concurrent 367 engagement of two circuits with opposing messages could compete to produce nicotine reinforcement, 368 and whether an imbalance between the two could lead to addiction. Indeed, this question may prove 369 critical when it comes to medical strategies aimed at smoking cessation. While the optogenetic strategies 370 used in this study are well suited to mimic the individual effects of a drug that also produces strong and 371 synchronized neuronal activity, the translational value of these effects is perhaps not to be sought in the 372 specific activation or inhibition of a given neuronal pathway, but rather in the functional imbalance that 373 this creates between the target structures of VTA neurons. Nevertheless, a detailed understanding of the 374 multiple pathways engaged in nicotine-evoked responses and of their respective behavioral contributions 375 can still help us to understand the mechanisms leading to nicotine addiction. In this respect, the activation 376 and inhibition processes which appear in VTA DA neurons as a consequence of systemic nicotine 377 injection call for further mechanistic studies, since they correspond to discrete neuronal circuits and 378 mediate distinct behavioral effects, both of which are relevant to the understanding of addiction. 
380 We are grateful to France Lam and the imaging platform facility (IBPS), the animal facilities (IBPS), Victor 381 Gorgievski for behavioral data acquisition, and Jérémie Naudé for technical and statistical advices. We 382 are grateful to Yulong Li laboratory (Peking University, Beijing) for providing us with GRAB ${ }_{D A}$ sensor 383 plasmids. We are grateful to Mélissa Desrosiers, Camille Robert, and AAV production facility of Paris 384 Vision Institute for viral production and purification.

385 This work was supported by Centre national de la recherche scientifique (CNRS UMR 8246), Institut 386 national de la santé et de la recherche médicale (Inserm U1130), Fondation pour la recherche médicale 387 (FRM DEQ2013326488 to PF, FRM FDT201904008060 to SM, FRM ECO201806006688 to JJ, FRM 388 SPF202005011922 to CS), French National Cancer Institute Grant TABAC-16-022 et TABAC-19-020 (to 389 PF), French state funds managed by Agence nationale de la recherche (ANR-16 Nicostress to PF, ANR39019 Vampire to FM), and LabEx Bio-Psy (to PF, and doctoral fellowship to CN), and LMR was supported 391 by a NIDA-Inserm Postdoctoral Drug Abuse Research Fellowship. PF and UM are members of LabEx 392 Bio-Psy.

\section{Author contributions:}

394 C.N., F.M. and P.F. designed the study. C.N., F.M. and P.F. analyzed the data. C.N. and F.M. performed 395 in vivo electrophysiological recordings. L.M.R, S.T. and S.V. contributed to in vivo electrophysiological 396 recordings. T.L.B. contributed to in vivo electrophysiological data analyses. S.M. designed, performed 397 and analyzed ex vivo patch-clamp recordings. C.N. performed stereotaxic injections (with the contribution 398 of S.M. and T.L.B.), fiber/cannula/catheter implantations and behavioral experiments. C.N., S.M., T.L.B., 399 I.C. and F.M. performed immunostaining experiments. M.C., C.S. and S.D. designed and performed 400 tetrode implantation, and signal analysis for in vivo recordings on freely moving animals. S.M., T.L.B. I.C. 401 M.C., J.J., C.S. and B.H. contributed to behavioral experiments. J.J. performed signal treatment and 402 analysis for fiber photometry experiments. R.D.C. and A.M. contributed to optogenetic experiments. D.D., 403 S.P. and U.M. provided viruses. U.M. provided ACNB2 KO mice. J.F.F. and V.D.G. contributed to 404 behavioral experiments and to design protocols. C.N., L.M.R. J.P.H., A.M., F.M. and P.F. wrote the 405 manuscript.

406 Declaration of Interests:

407 Authors declare no competing financial interests. 


\section{Figure legends}

410 Figure 1: Nicotine injection evokes opposing responses in distinct VTA DA neuron populations

411 (A) Intravenous (IV) injections of nicotine (Nic, $30 \mu \mathrm{g} / \mathrm{kg}$ ) induce activation or inhibition of distinct VTA DA 412 neurons in anesthetized mice (representative recordings). Post-recording identification of neurobiotin

413 (NB)-labeled VTA DA neurons by immunofluorescence ( $\mathrm{TH}=$ tyrosine hydroxylase, NB = streptavidin414 AMCA against neurobiotin). (B) Response density after IV injection of either saline (Sal, grey, $n=233$ ) or 415 nicotine (Nic, black, $n=245$ ) expressed as percentage of firing frequency variation induced by the injection 416 (Kolmogorov-Smirnov test, ${ }^{* * *} p<0.001$ ). (C) Time course for the average change in firing frequency 417 upon nicotine injection for activated (Nic+ in red, $n=155$, maximum variation $+33.75 \pm 52.52 \%$ ) and 418 inhibited (Nic- in blue, $n=88$, minimum variation $-35.43 \pm 23.63 \%$ ) VTA DA neurons. (D) Firing rate 419 variation $(\Delta)$ from baseline (Bas) induced by Nic or Sal injection in nicotine-activated and nicotine-inhibited 420 DA neurons. Comparison between mean firing rate during baseline and maximal firing rate after injection 421 for activated neurons, and between mean firing rate during baseline and minimal firing rate after injection 422 for inhibited neurons (paired Wilcoxon test, ${ }^{* * *} p<0.001$, ns $p>0.05$ ), and comparisons between saline423 induced and nicotine-induced firing rate variations (Wilcoxon test, ${ }^{* * *} p<0.001$ ). Mean scores are 424 represented in black and individual scores in grey. (E) Dose-response curves in Nic+ (red) and Nic- (blue) 425 DA neurons. Responses to different doses of nicotine $(0,10,15,30,60,90 \mu \mathrm{g} / \mathrm{kg}$ with $\mathrm{n}=48,9,27,51$, 42633,17 for activated neurons, and $n=11,3,3,12,9,5$ for inhibited neurons) are expressed as percentage 427 of variation from baseline. Neurons are classified as activated or inhibited on the basis of their response 428 to the injection at at least $30 \mu \mathrm{g} / \mathrm{kg}$ nicotine (one-way ANOVA dose effect, Nic+ $F_{(5,179)}=7.54{ }^{* * *} p<0.001$ 429 and Nic- $\left.F(5,37)=4.78,{ }^{* *} p=0.002\right)$. (F) Time course for the average change in firing frequency upon saline 430 (grey, $n=16$ ) or nicotine injection for Nic+ (red, $n=8$ ) and Nic- (blue, $n=8$ ) VTA DA neurons recorded 431 with tetrodes in freely moving mice with examples of traces for a Nic+ (red) and a Nic- (blue) neuron. (G) 432 Localization of NB-labeled, Nic+ and Nic- DA neurons ( $n=243)$, positioned on the Paxinos atlas at bregma $433-3.3 \mathrm{~mm}$. Nic- neurons had a more medial distribution within the VTA than Nic+ neurons (Wilcoxon test, $434{ }^{* * *} p<0.001$ ), but neither antero-posterior (Wilcoxon test, $p=0.4$ ) nor dorso-ventral (Wilcoxon test, $p=$ 4350.56 ) differences in their distribution were observed. 
438 (A) Retrobeads (RB) were injected in the nucleus accumbens (NAc, injection in the lateral shell (LSh) + medial shell (MSh) + core), and in vivo recordings of VTA DA neuron responses to an IV nicotine injection were performed on anesthetized mice. Post hoc identification of NAc-projecting DA neurons by immunofluorescent co-labeling of tyrosine hydroxylase (TH), neurobiotin (NB) and retrobeads (RB). (B) Localization of NB-labeled DA neurons ( $N B+T H+, n=49$ ) following RB injection ( $\bullet R B+, \circ R B-)$ into the NAc. Red and blue colors denote nicotine-activated (Nic+) and nicotine-inhibited (Nic-) neurons, respectively. (RB+Nic+, $n=28 ; R B+$ Nic-, $n=2 ; R B-N i c+, n=4 ; R B-$ Nic-, $n=15)$. (C) Left: Percentage and number of Nic+ (red) and Nic- (blue) cells among NAc-projecting DA neurons (RB+, top) or non-RBlabeled neurons (RB-, bottom), with mean change in firing frequency in response to IV injection of either nicotine (red or blue, $30 \mu \mathrm{g} / \mathrm{kg}$ ) or saline (black). Right: Firing rate variation $(\Delta)$ from baseline (Bas) induced by nicotine (Nic) injection in RB+ (mean $\Delta=+0.52 \mathrm{~Hz}$ ) or RB- (mean $\Delta=-0.61 \mathrm{~Hz}$ ) or RB- DA neurons following RB injection into the NAc. (Comparison between mean firing rate during baseline and maximum/minimum firing rate after injection: paired Wilcoxon test ${ }^{* * *} p(R B+)<0.001,{ }^{*} p(R B-)=0.017$, comparison between nicotine-induced firing rate variation evoked in RB+ and RB- DA neurons: Wilcoxon test ${ }^{* * *} p<0.001$. Mean scores are represented in black, and individual scores in red or blue). (D) Same as in (A) but with RB injected in the amygdala (Amg: injection in central nucleus (CeA) + basolateral amygdala (BLA)). (E) Localization of $N B+D A$ neurons $(N B+T H+, n=52)$ following $R B$ injection into the Amg (RB+ Nic+, $n=3$; RB+ Nic-, $n=19 ;$ RB- Nic+, $n=23 ;$ RB- Nic-, $n=7$ ). (F) Left: Percentage and number of Nic+ (red) and Nic- (blue) cells among Amg-projecting DA neurons (RB+, top) or non-RBlabeled neurons (RB-, bottom). Right: Firing rate variation $(\Delta)$ from baseline (Bas) induced by nicotine (Nic) injection in RB+ (mean $\Delta=-0.32 \mathrm{~Hz}$ ) or RB-(mean $\Delta=+0.79 \mathrm{~Hz}$ ) DA neurons following RB injection into the Amg. (Comparison between mean firing rate during baseline and maximum/minimum firing rate after injection: paired Wilcoxon test ${ }^{*} p(R B+)=0.027,{ }^{* *} p(R B-)=0.002$, comparison between nicotineinduced firing rate variation evoked in RB+ and RB- DA neurons: Wilcoxon test $\left.{ }^{* * *} p<0.001\right)$. (G) Density of responses evoked by nicotine in NAc-projecting (gold) and Amg-projecting (purple) DA neurons. Responses expressed as percentage of firing variation induced by nicotine (Kolmogorov-Smirnov test ${ }^{* * *}$ $p<0.001)$. (H) AAV-mediated delivery of the genetically encoded GPCR-activation-based-DA sensor $\left(G R A B_{D A}\right)$ in the BLA and the NAcLSh of wild-type (WT) mice. One optic fiber was implanted in the BLA of one brain hemisphere and a second fiber was implanted in the NAcLSh of the other hemisphere. Examples of fluorescence variation of GRABDA expression (as $\triangle F / F$ ) induced by IV Nic or Sal injection and fiber implantation sites (Left) in the BLA and (Right) in the NAcLSh with post hoc verification of both implantations. (I) (Left) Mean fluorescence variation of GRAB ${ }_{D A}$ (expressed as $\triangle F / F$, transparent curves (mean $\Delta \mathrm{F} / \mathrm{F}$ ), bold curves (kernel fit of $\Delta \mathrm{F} / \mathrm{F})$ ) induced by saline (grey) or nicotine IV injection (30 $\mu \mathrm{g} / \mathrm{kg}$ ) in freely moving mice recorded by fiber photometry in the NAcLSh (gold, $n=8$ injections in 6 mice) and the BLA (purple, $n=7$ injections in 6 mice). (Right) Difference in peak $\Delta F / F$ between nicotine and saline (Paired Student's t-test, ${ }^{* * *} p<0.001$ and ${ }^{*} p=0.011$ for NAcLSh and BLA; difference in $\Delta F / F$ (Nicotine Saline) between NAcLSh and BLA, Student's t-test, ${ }^{* * *} p<0.001$ ) 

and nicotine-induced anxiety-like behavior.

(A) Nicotine (Nic, $0.5 \mathrm{mg} / \mathrm{kg}$ ) or saline (Sal) was injected intraperitoneally (IP) 1 minute before the 9minute elevated O-maze (EOM) test. Nic injection in a group of wild-type (WT) mice $(n=21)$ decreased the time they spent in the open arms of the EOM compared to the group injected with Sal $(n=23)$ (twoway RM ANOVA treatment $x$ time interaction $F_{(2,84)}=5.37$, ${ }^{* *} p=0.006$, main effect of time $F_{(2,84)}=3.84$, * $p=0.025$; post hoc Wilcoxon test with Bonferroni corrections: * $p$ ( 3 vs 9 minutes $)=0.03 ; p(3$ vs 6 minutes $)=0.1 ; p\left(6\right.$ vs $\left.9^{\prime}\right)=0.2 ;$ post hoc Wilcoxon test Sal vs Nic at 9 minutes, $\left.{ }^{* * *} p<0.001\right)$. (B) Mice implanted with intracranial (IC) bilateral guide cannulas were injected either with Sal or with Nic (100 ng in $100 \mathrm{nl}$ infusion) over 1 minute before the 9-minute EOM test. The Nic-injected mice $(n=7)$ spent less time in the open arms over time, but not the control mice $(n=6)$ (two-way RM ANOVA treatment $x$ time interaction $F_{(2,22)}=9.66{ }^{* * *} p<0.001$, main effect of time ${ }^{* * *} p<0.001$; post hoc Student's t-test with Bonferroni corrections: ${ }^{* * *} p$ ( 3 vs 9 minutes) $<0.001 ;{ }^{*} p$ ( 3 vs 6 minutes) $=0.025 ;{ }^{*} p$ ( 6 vs 9 minutes) $=$ 0.02 ; post hoc Student's t-test Sal vs Nic at 9 minutes, $p=0.054$ ). (C) Top left: Representative juxtacellular recording traces of VTA DA neurons in mice lacking the $\beta 2 \mathrm{nAChR}$ subunit $\left(\beta 2^{--}\right)$and in $\beta 2^{-- \text {-vectorized }}$ mice, in which the $\beta 2$ subunit has been virally re-expressed together with a GFP marker in the VTA ( $\beta 2-$ Vec). Bottom left: Individual and mean responses (expressed as percentage of firing frequency variation) indicate that there were no Nic-evoked responses in VTA DA neurons of $\beta 2$ - - mice $(n=46$ cells from 12 mice), and that both Nic-evoked activation ( $n=51$ cells from 18 mice) and inhibition ( $n=39$ cells from 19 mice) of VTA DA neurons were restored in $\beta 2^{-1-V e c ~ m i c e . ~ T o p ~ r i g h t: ~ I m m u n o f l u o r e s c e n c e ~ f o r ~ T H ~ a n d ~ G F P ~}$ on $\beta 2-$-Vec mice. Bottom right: Cumulative distribution of Nic-evoked response amplitude of VTA DA neurons in $\beta 2-$ mice ( $n=46$ cells from 12 mice, grey) and $\beta 2--V e c$ mice ( $n=90$ cells from 24 mice, black) (Kolmogorov-Smirnov test ${ }^{* *} p=0.008$ ). Bar plots show the maximum firing variation induced by Nic (filled bars) and saline (unfilled bars) in the two groups. Nic injection did not alter the firing frequency of VTA DA neurons in $\beta 2 \%$ mice, however it induced a significant increase (mean $12.45 \pm 13.37$ ) or decrease (mean $-13.16 \pm 16.31)$ in the firing frequency of VTA DA neurons in $\beta 2$ Vec mice compared to saline ${ }^{* * *} p<$ $0.001,{ }^{* * *} p<0.001$ ) or $\left({ }^{* * *} p<0.001,{ }^{* *} p=0.005\right.$ ) (Wilcoxon paired test with Bonferroni corrections) (D) EOM test after IP Nic injection $(0.5 \mathrm{mg} / \mathrm{kg})$ in a control group of $\beta 2--$ mice some of which were shamtransduced with GFP in the VTA (see methods, $\beta 2--n=23)$ and in $\beta 2-$ Vec mice $(n=18)$. Re-expression of $\beta 2$ subunit in the VTA ( $\beta 2--V$ ec) restored the nicotine-evoked anxiogenic effects in the EOM test, which was absent in the $\beta 2^{-1}$ mice (two-way RM ANOVA interaction treatment $x$ time $F_{(2,78)}=3.43,{ }^{*} p=0.04$, main time effect $F_{(2,78)}=6.87,{ }^{* *} p=0.002$; post hoc Student's t-test with Bonferroni corrections: ${ }^{* *} p(3$ vs 9 minutes $)=0.003 ;{ }^{*} p(3$ vs 6 minutes $)=0.03 ; p(6$ vs 9 minutes $)=0.2 ;$ post hoc Student's t-test $\beta 2-$ and $\beta 2-$ GFP vs $\beta 2-$ Vec mice at 9 minutes, $p=0.06$ ). 
509 Figure 4: Inhibition of BLA- projecting DA neurons drive anxiety-like behavior, but not place 510 preference.

511 (A) Left: AAVs for Cre-dependent expression of Jaws (orange, $n=18$ ) or YFP (green, $n=19$ ) were 512 injected into the VTA of DAT-Cre mice, and optical fibers were placed in the BLA. Right: Percentage of 513 time spent in the EOM open arms for mice stimulated continuously at $520 \mathrm{~nm}$ over a 5 -minute period (ON) 514 in the BLA (Two-way RM ANOVA time $x$ opsin interaction $\mathrm{F}_{(2,70)}=3.32$, * $p=0.04$; post hoc Student's t515 test for Jaws vs YFP mice: * $p(O N)=0.04$; post hoc Student's t-test with Bonferroni corrections for Jaws 516 mice, ${ }^{*} p(5$ vs 10 minutes $)=0.01,{ }^{*} p(10$ vs 15 minutes $\left.)=0.02\right)$. (B) Left: AAVs for Cre-dependent 517 expression of CatCh (blue, $n=18$ ) or YFP (green, $n=19$ ) were injected into the VTA of DAT-Cre mice, 518 and optical fibers were placed in the BLA. Right: Percentage of time spent in the EOM open arms for mice 519 stimulated at $470 \mathrm{~nm}$ over a 5 -minute period (ON) at $10 \mathrm{~Hz}, 5$ ms-pulses in the BLA (Two-way RM ANOVA 520 main effect of time $F_{(2,70)}=4.41$, ${ }^{*} p=0.02$, time $x$ opsin interaction $F_{(2,70)}=4.43$, ${ }^{*} p=0.015$; post hoc 521 Student's t-test for CatCh vs YFP mice, ${ }^{* *} p(O N)=0.009$, post hoc Student's t-test with Bonferroni 522 corrections for CatCh mice, ${ }^{* *} p(5$ vs 10 minutes $)=0.001 ;{ }^{*} p(10$ vs 15 minutes $\left.)=0.01\right)$. (C) Left: AAVs 523 for Cre-dependent expression of CatCh (blue, $n=13$ ) or YFP (green, $n=9$ ) were injected into the VTA of 524 DAT-Cre mice, and optical fibers were placed in the BLA. Nicotine (Nic, $0.5 \mathrm{mg} / \mathrm{kg}$ ) was injected 525 intraperitoneally (IP) 1 minute before the 9-minute elevated O-maze (EOM) test. Right: Percentage of 526 time spent in the EOM open arms for mice stimulated in the BLA throughout the test at $10 \mathrm{~Hz}, 5$-ms light527 pulses after IP nicotine injection (Two-way RM ANOVA main effect of time $F_{(2,40)}=4.92,{ }^{*} p=0.01$, time $528 x$ opsin interaction $\mathrm{F}_{(2,40)}=3.74,{ }^{*} p=0.03$; post hoc Student's t-test for CatCh vs YFP mice at 9 minutes $529{ }^{* *} p=0.006$ ). (D) Preference score in a 20 min-real-time place preference test (RTPP) defined by the $\%$ 530 of time spent in the compartment where animals are photo-stimulated compared to the compartment 531 where they are not (ON-OFF). Optical inhibition of the VTA-BLA pathway (orange, $n=17$ mice) induced 532 online place avoidance compared to the control mice (YFP, green, $n=20$ ) (Student's t-test, ${ }^{*} p=0.017$ ). 533 Mice with optical activation of the VTA-BLA pathway (blue, $n=12$ ) did not display any difference compared 534 to the control mice (YFP, green, $n=13$ ) (Student's t-test, $p=0.5)$. 


\section{Figure 5: Activation of NAcLSh-projecting VTA DA neurons drives real-time place preference}

536 behavior, but has no effect on anxiety-like behavior

537 (A) Left: AAVs for Cre-dependent expression of CatCh (blue, $n=13$ ) or YFP (green, $n=14$ ) were injected 538 into the VTA of DAT-Cre mice, and optical fibers were placed in the NAcLSh. Right: Percentage of time

539 spent in the EOM open arms for mice stimulated at $470 \mathrm{~nm}$ over a 5 -minute period (ON) at $10 \mathrm{~Hz}, 5 \mathrm{~ms}$ 540 pulses in the NAcLSh (Two-way RM ANOVA no time or opsin effect, nor interaction $F_{(2,50)}=0.8, p=0.5$ ).

541 (B) Left: AAVs for Cre-dependent expression of Jaws (orange, $n=12$ ) or YFP (green, $n=12$ ) were 542 injected into the VTA of DAT-Cre mice, and optical fibers were placed in the NAcLSh. Right: Percentage 543 of time spent in the EOM open arms for mice stimulated continuously at $520 \mathrm{~nm}$ over a 5-minute period $544(\mathrm{ON})$ in the NAcLSh (Two-way RM ANOVA no time or opsin effect, nor interaction $F_{(2,44)}=0.16, p=0.8$ ). 545 (C) Left: AAVs for Cre-dependent expression of Jaws (orange, $n=11$ ) or YFP (green, $n=12$ ) were 546 injected into the VTA of DAT-Cre mice, and optical fibers were placed in the NAcLSh. Nicotine (Nic, 0.5 $547 \mathrm{mg} / \mathrm{kg}$ ) was injected intraperitoneally (IP) 1 minute before the 9-minute elevated O-maze (EOM) test. 548 Right: Percentage of time spent in the EOM open arms for mice stimulated continuously in the NAcLSh 549 throughout the test after IP nicotine injection (Two-way RM ANOVA main time effect $F_{(2,42)}=12.6$, ${ }^{* * *} p<$ $550 \quad 0.001$, opsin effect $F_{(1,21)}=5.08$, * $p=0.03$, no interaction $F_{(2,42)}=0.55, p=0.6$ ). (D) Preference score in 551 a 20 min-RTPP test defined by the \% of time spent in the compartment where animals are photo552 stimulated compared to the compartment where they are not (ON-OFF). Mice with optical inhibition of the 553 VTA-NAcLSh pathway (orange, $n=12$ ) did not display any difference compared to the control mice (YFP, 554 green, $n=12$ ) (Student's t-test, $p=0.5$ ). Optical activation of the VTA-NAcLSh pathway (blue, $n=13$ ) 555 induced online place preference compared to the control mice (YFP, green, $n=14$ ) (Student's t-test, ${ }^{*} p$ $556=0.04)$. 


\section{LEAD CONTACT}

559 Further information and requests for resources and reagents should be directed to and will be fulfilled by

560 the Lead Contact, Philippe Faure (phfaure@gmail.com) or Fabio Marti (fabio.marti@upmc.fr).

\section{MATERIALS AVAILABILITY}

562 This study did not generate new unique reagents.

\section{DATA AND CODE AVAILABILITY}

564 All the data are available from the corresponding authors upon request.

565

566

567

568

569

570

571

572

573

574

575

576

577

578

579

580

581

582

583

584

585

586

587

588

589

\section{EXPERIMENTAL MODEL AND SUBJECT DETAILS}

Wild-type (WT) C57BL/6J (Janvier Labs, France), ACNB2 KO ( $\beta 2^{---}$) and DATiCRE (DAT-Cre) male mice, weighing 25-35 grams, were used in this study. $\beta 2$-- mice were generated using standard homologous recombination procedures. Founders were backcrossed onto a C57BL/6J background for a least 20 generations and bred on site. DATICRE mice were provided by François Tronche (IBPS Paris, France). They were bred on site and genotyped as described (Turiault et al., 2007).

Mice were kept in an animal facility where temperature $\left(20 \pm 2{ }^{\circ} \mathrm{C}\right)$ and humidity were automatically monitored, and a circadian light-dark cycle of 12/12 hours was maintained. All experiments were performed on 8-to-16-week-old mice. All experiments were performed in accordance with the recommendations for animal experiments issued by the European Commission directives 219/1990, 220/1990 and 2010/63, and approved by Sorbonne University.

\section{METHOD DETAILS}

\section{Viral production}

AAV vectors were produced as previously described (Khabou et al., 2018) using the co-transfection method, and purified by iodixanol gradient ultracentrifugation (Choi et al., 2007). AAV vector stocks were titrated by quantitative PCR (qPCR) (Aurnhammer et al., 2012) using SYBR Green (Thermo Fischer Scientific). Lentiviruses were prepared as previously described (Maskos et al., 2005; Tolu et al., 2013), with a titer of either $380 \mathrm{ng}$ of p24 protein per $\mu \mathrm{L}$ or $764 \mathrm{ng} / \mu \mathrm{L}$ for the AChR $\beta 2$-expressing vector, and $150 \mathrm{ng}$ of p24 protein per $\mu \mathrm{L}$ or $361 \mathrm{mg}$ per $2 \mu \mathrm{L}$ for GFP-expressing vector.

\section{Drugs}

The nicotine (Nic) used for all experiments is a nicotine hydrogen tartrate salt (Sigma-Aldrich, USA). For juxtacellular recordings, we performed an intravenous injection (IV) of Nic at a dose of $30 \mu \mathrm{g} / \mathrm{kg}(4.16$ $\mathrm{mg} / \mathrm{kg}$, free base) or saline solution $\left(\mathrm{H}_{2} \mathrm{O}\right.$ with $\left.0.9 \% \mathrm{NaCl}\right)$. For the behavioral test, in elevated O-maze (EOM) or open-field (OF), mice were injected intra-peritoneally (IP) with Nic at $0.5 \mathrm{mg} / \mathrm{kg}, 1$-minute before the test. For intra-cranial (IC) experiments in EOM, saline solution or 100ng of Nic tartrate, in a volume of 
$590100 \mathrm{nl}$, were infused over 1 minute before the beginning of the test. All solutions were prepared in the 591 laboratory.

\section{Stereotaxic surgeries}

593 For virus and RB injections, intracranial cannulas, fibers, catheters and micro-drive implantations, mice 594 were anesthetized with a gas mixture of oxygen $(1 \mathrm{~L} / \mathrm{min})$ and $3 \%$ isoflurane (Vetflurane $囚$, Virbac) for the 595 induction of anesthesia, and then placed in a stereotaxic frame (David Kopf) maintained under anesthesia 596 throughout the surgery at $1 \%$ isoflurane. A local anesthetic (100 $\mu \mathrm{L}$ Lurocaine $囚)$ was applied at the 597 location of the scalp incision or the catheter implant before the procedure. At the end of the surgery, 0.1 $598 \mathrm{~mL}$ of buprenorphine (Buprecare $囚, 1 \mathrm{mg} / \mathrm{kg}$ ) was injected subcutaneously to prepare awakening.

\section{Retrobead injection}

600 Green fluorescent retrograde tracer, retrobeads (RB, LumaFluor Inc., Naples, FL), were injected (200 nL 601 per site, $0.1 \mu \mathrm{L} / \mathrm{min}$ ) in WT animals either in the NAc (NAc lateral shell NAcLSh: bregma $1.45 \mathrm{~mm}$, lateral $6021.75 \mathrm{~mm}$, ventral $4.0 \mathrm{~mm}$; NAc medial shell NAcMSh: bregma $1.78 \mathrm{~mm}$, lateral $0.45 \mathrm{~mm}$, ventral $4.1 \mathrm{~mm}$; 603 NAc core: bregma $1.55 \mathrm{~mm}$, lateral $1.0 \mathrm{~mm}$, ventral $4.0 \mathrm{~mm}$ ) or in the Amg (BLA: bregma $-1.61 \mathrm{~mm}$, lateral $6043.18 \mathrm{~mm}$, ventral $4.7 \mathrm{~mm}$; CeA: bregma $-0.78 \mathrm{~mm}$, lateral $2.3 \mathrm{~mm}$, ventral $4.8 \mathrm{~mm}$ ) with a $10 \mu \mathrm{L}$ Hamilton 605 syringe (Hamilton) coupled with a polyethylene tubing to a $36 \mathrm{G}$ injection cannulas (Phymep). Note that 606 these empirically derived stereotaxic coordinates do not precisely match those given in the mouse brain 607 atlas (Paxinos and Franklin, 2004), which we used as references for the injection-site images. To enable 608 retrograde transport of the RB into the somas of midbrain DA neurons, we waited for an adequate time to 609 perform the electrophysiology experiments, depending on the injection zone: 3 weeks after injection into 610 the NAc and 2 weeks after injection into the Amg.

\section{Intracranial infusion}

612 Bilateral guide cannulas (Bilaney) were implanted in the VTA (bregma $3.1 \mathrm{~mm}$, lateral $0.5 \mathrm{~mm}$, ventral 4.3 $613 \mathrm{~mm}$ ) of WT mice under anesthesia 1 week before the EOM experiment, in order to enable local infusion 614 of drugs. Before each experiment session a double injection cannula ( $4.5 \mathrm{~mm}$ length, $1 \mathrm{~mm}$ interval) was 615 inserted into the implanted bilateral guide cannulas (length under pedestal $4.0 \mathrm{~mm}$ ), $0.5 \mathrm{~mm}$ beyond the 616 tip of the guide cannulas. The day of the experiment, the cannulas were connected to a multi-syringe 617 pump (Univentor) allowing saline or nicotine (100 ng) injection over 1 minute (injected volume of $100 \mathrm{~nL}$ ).

618 Virus injection and optogenetic experiments

619 For lentiviral re-expression of the $\beta 2$ subunit, we performed bilateral injections of $1 \mu \mathrm{L}$ of PGK- $\beta 2-I R E S-$ 620 GFP ( $\beta 2^{--V e c}$ mice) or sham PGK-IRES-GFP into the VTA of $\beta 2^{--}$mice (coordinates from bregma 3.1 $621 \mathrm{~mm}$, lateral $0.5 \mathrm{~mm}$, ventral $4.5 \mathrm{~mm}$ ).

622 To perform DA neuron-specific optogenetic experiments, intracranial (IC) injections were performed 623 bilaterally into the VTA (bregma $3.1 \mathrm{~mm}$, lateral $0.5 \mathrm{~mm}$, ventral $4.5 \mathrm{~mm}$ ) of 8-week-old DAT-Cre mice, in 624 which Cre recombinase expression is restricted to DA neurons without disrupting endogenous dopamine 625 transporter (DAT) expression (Turiault et al., 2007; Zhuang et al., 2005), with $0.5 \mu \mathrm{L}$ of AAV per 626 hemisphere (AAV5.EF1 .DIO.CatCh.YFP $2.46 \mathrm{e}^{12}$ or $6.53 \mathrm{e}^{13} \mathrm{vg} / \mathrm{mL}$ used in the BLA and 627 AAV9.EF1 $\alpha . D I O . h C h R 2 . Y F P ~ 9.59 \mathrm{e}^{13} \mathrm{vg} / \mathrm{mL}$ used in the NAcLSh, AAV5.EF1 $\alpha . D I O . J a w s . e G F P 1.16 \mathrm{e}^{13}$ $628 \mathrm{vg} / \mathrm{mL}$, AAV5.EF1 $\alpha$.DIO.YFP $6.89 \mathrm{e}^{13}$ or $9.10 \mathrm{e}^{13} \mathrm{vg} / \mathrm{mL}$ ). A double-floxed inverse open reading frame 629 (DIO) allowed restraining to VTA DA neurons the expression of CatCh, a channelrhodopsin mutant with 
630 enhanced light sensitivity and $\mathrm{Ca}^{2+}$ permeability (Kleinlogel et al., 2011) for activation, or Jaws a red631 shifted cruxhalorhodopsin Jaws (Chuong et al., 2014) for inhibition (Figure S9). Optical fibers (200 $\mu \mathrm{m}$ 632 core, $N A=0.39$, Thor Labs) coupled to a zirconia ferule $(1.25 \mathrm{~mm})$ were implanted bilaterally in the 633 different target sites of the VTA (coordinates for BLA implantation: bregma $-1.6 \mathrm{~mm}$, lateral $3.18 \mathrm{~mm}$, 634 ventral $4.5 \mathrm{~mm}$ ) (coordinates for NAcLSh implantation: bregma $1.5 \mathrm{~mm}$, lateral $1.75 \mathrm{~mm}$, ventral 3.90 $635 \mathrm{~mm}$ ), and fixed to the skull with dental cement (SuperBond, Sun medical). An ultra-high-power LED (470 $636 \mathrm{~nm}$ for Catch, $520 \mathrm{~nm}$ for Jaws, Prizmatix) coupled to a patch cord (500 $\mu \mathrm{m}$ core, NA = 0.5, Prizmatix) 637 was used for optical stimulation (output intensity of $10 \mathrm{~mW}$, frequency of $10 \mathrm{~Hz}, 5 \mathrm{~ms}$-pulse for CatCh, 638 continuous stimulation at $520 \mathrm{~nm}$ for Jaws).

To perform non-conditional expression in different subnuclei of the amygdala (Amg), an AAV2-CAG-JawsGFP $\left(1.45 \mathrm{e}^{12} \mathrm{ng} / \mu \mathrm{L}\right)$ or AAV2-7m8-CAG-GFP $\left(5.70 \mathrm{e}^{12} \mathrm{ng} / \mu \mathrm{L}\right)$ were injected bilaterally into the VTA (same coordinates as previously indicated) of distinct groups of 8-week-old WT mice. Optical fibers were bilaterally implanted in those mice either in the basolateral amygdala (BLA: bregma $-1.6 \mathrm{~mm}$, lateral 3.18 $\mathrm{mm}$, ventral $4.5 \mathrm{~mm}$ ) or in the central amygdala (CeA: bregma $-0.78 \mathrm{~mm}$, lateral $2.3 \mathrm{~mm}$, ventral $4.8 \mathrm{~mm}$ ). To perform non-conditional expression in the NAc medial shell (NAcMSh), a AAV5.hSyn.hChR2(H134R).eYFP

$\left(2.4 \mathrm{e}^{13}\right)$ or AAV5.hsyn.Jaws.KGC.GFP.ER2

$\left(1.3 e^{13}\right)$ or AAV5.hSyn.eGFP.WPRE.bGH $\left(2.2 \mathrm{e}^{13}\right)$ was injected bilaterally in the VTA of distinct groups of 8 weekold WT male mice. Optical fibers were bilaterally implanted in those mice in the NAcMSh with $12^{\circ}$ angle (NAcMSh: bregma $1.5 \mathrm{~mm}$, lateral $1.5 \mathrm{~mm}$, ventral $4.5 \mathrm{~mm}$ ).

All experiments were conducted at least 4 weeks after viral injection, to enable expression of the different constructs. The optical stimulation cable was plugged onto the ferule during all experimental sessions when on purpose, to habituate the animals and control for latent experimental effects.

\section{Fiber photometry experiments}

655 8-week-old WT mice were injected with $0.5 \mu$ of AAV-hSyn-GRAB ${ }_{\text {DA2m }}\left(1.23 \mathrm{e}^{14} \mathrm{vg} / \mathrm{mL}\right)$ in the BLA 656 (bregma - $1.61 \mathrm{~mm}$, lateral $3.18 \mathrm{~mm}$, ventral $4.7 \mathrm{~mm}$ ) or NAcLSh (bregma $1.5 \mathrm{~mm}$, lateral $1.55 \mathrm{~mm}$, ventral $6573.95 \mathrm{~mm}$ ). Optical fibers ( $200 \mu \mathrm{m}$ core, $\mathrm{NA}=0.39$, Thor Labs) coupled to a stainless-steel ferule (1.25 $658 \mathrm{~mm}$ ) were implanted after virus injection at the same coordinates, and fixed to the skull with dental cement 659 (SuperBond, Sun medical). Two weeks after surgeries, animals begin a habituation period to the plastic 660 cylinder used for photometry recordings.

661 Before the measurements, the animals were implanted in one of the tail veins with a catheter (30G needle connected to PE10 tubing). Venous return and the absence of tail swelling after a saline injection ensured

663 a good positioning of the catheter, which was then glued and taped to the animal's tail. During awakening, 664 the mouse was placed into a $7 \mathrm{~cm}$-diameter plastic cylinder from which its tail can protrudes on the outside 665 via a hole (see schematic on Figure S2B).

666 Fluorescence measurements of DA levels in the NAcLSh and BLA were recorded using a Doric Lenses 667 1-site 2-color fiber photometry system. The fiber photometry console was connected to the LED driver to 668 control connectorized LED in Lock-in mode (CLED $465 \mathrm{~nm}$ modulated at $220.537 \mathrm{~Hz}$ ) that was connected 669 to its port on the Mini Cube (FMC4_AE(405)_E(460-490)_F(500-550)_S) through an optic patch cord 670 (MFP_200/220/LWMJ-0.37_1m_FC-FC_T0.20). Light stimulation and recorded fluorescence were 671 transmitted through an optical fiber (FT400EMT, $400 \mu \mathrm{m}$ core, NA $=0.39$, Thorlabs) connected both to 
672 the animal's implanted optical fiber via a zirconia sleeve and to the sample (S) port on the Mini Cube. 673 Finally, the photoreceiver converting recorded light to electrical signals (AC Low setting, New Focus 2151

674 Visible Femtowatt Photoreceiver, New Focus, San Jose, CA, USA) was connected to the Mini Cube 675 through an optic path cord ( $600 \mu \mathrm{m}$ core, NA = 0.48, FC-FC, Doric Lenses) fitted on a fiber optic adapter 676 (Doric Lenses) and to the fiber photometry console. Signal was acquired through Doric Neuroscience 677 Studio software (version 5.2.2.5) with a sampling rate of $12.0 \mathrm{kS} / \mathrm{s}$ (kilosamples per second) and a low678 pass filter with a cutoff frequency of $12.0 \mathrm{~Hz}$.

679 We assessed changes in DA levels in NAcLSh or BLA in response to saline or nicotine injection in the tail 680 vein of the animal. After catheter implantation, the animal recovers in the plastic cylinder for $30 \mathrm{~min}$. We 681 then started to record after at least $3 \mathrm{~min}$ baseline, $5 \mathrm{~min}$ after saline injection, $15 \mathrm{~min}$ after $30 \mu \mathrm{g} / \mathrm{kg}$ or $68260 \mu \mathrm{g} / \mathrm{kg}$ nicotine injection. After the session, mice were re-anesthetized to carefully remove the catheter 683 and were allowed to rest for one day before the next recording session.

\section{In vivo electrophysiology on anesthetized mice}

685 Mice were deeply anesthetized with an IP injection of chloral hydrate (8\%), $400 \mathrm{mg} / \mathrm{kg}$, supplemented as 686 required to maintain optimal anesthesia throughout the experiment. The scalp was opened and a hole 687 was drilled in the skull above the location of the VTA. Intravenous administration of saline or nicotine $688(30 \mu \mathrm{g} / \mathrm{kg})$ was carried out through a catheter (30G needle connected to polyethylene tubing PE10) 689 connected to a Hamilton syringe, into the saphenous vein of the animal. For multiple doses of nicotine, 690 mice received first a dose of $30 \mu \mathrm{g} / \mathrm{kg}$ and then one to four subsequent injections of nicotine at different 691 doses, either 10, 15, 60 and/or $90 \mu \mathrm{g} / \mathrm{kg}$ (pseudo-randomly administrated). Extracellular recording 692 electrodes were constructed from $1.5 \mathrm{~mm}$ outer diameter / $1.17 \mathrm{~mm}$ inner diameter borosilicate glass 693 tubing (Harvard Apparatus) using a vertical electrode puller (Narishige). The tip was broken straight and clean under microscopic control to obtain a diameter of about $1 \mu \mathrm{m}$. The electrodes were filled with a $0.5 \% \mathrm{NaCl}$ solution containing $1.5 \%$ of neurobiotin ${ }^{\circledR}$ tracer (VECTOR laboratories) yielding impedances of 6-9 M $\Omega$. Electrical signals were amplified by a high-impedance amplifier (Axon Instruments) and monitored audibly through an audio monitor (A.M. Systems Inc.). The signal was digitized, sampled at 25

$698 \mathrm{kHz}$, and recorded on a computer using Spike2 software (Cambridge Electronic Design) for later analysis. 699 The electrophysiological activity was sampled in the central region of the VTA (coordinates: between 3.1 700 to $4 \mathrm{~mm}$ posterior to bregma, 0.3 to $0.7 \mathrm{~mm}$ lateral to midline, and 4 to $4.8 \mathrm{~mm}$ below brain surface). 701 Individual electrode tracks were separated from one another by at least $0.1 \mathrm{~mm}$ in the horizontal plane. 702 Spontaneously active DA neurons were identified based on previously established electrophysiological criteria (Grace and Bunney, 1984b; 1984a; Ungless and Grace, 2012). After recording, nicotine-responsive cells were labelled by electroporation of their membrane: successive currents squares were applied until the membrane breakage, to fill the cell soma with neurobiotin contained into the glass pipet (Pinault 1996). To be able to establish correspondence between neurons responses and their localization in the VTA, we labeled one type of response per mouse: solely activated neurons or solely inhibited neurons, with a limited number of cells per brain (1 to 4 neurons maximum, 2 by hemisphere), always with the same concern of localization of neurons in the VTA.

\section{In vivo electrophysiology on freely moving animals}

711 Micro-drive and electrodes: Hand-made poly-electrodes (bundle of 8 electrodes: "octrodes") were 712 obtained by twisting eight polyimide-insulated $17 \mu \mathrm{m}$ Nickel-Chrome wires (A-M SYSTEMS, USA). The 713 use of eight channels relatively close to each other allows for a better discrimination of the different 
neurons. Before implantation and recording, the octrodes were cut at suitable length and plated with a solution of platinum (platinum black plating solution, Neuralynx; Bozeman, USA) and poly-ethylene glycol (1 $\mathrm{mg} / \mathrm{mL}$ ) (25\% platinum - 75\% PEG) to lower their impedance to 200-500 kOhms and improve the signal-to-noise ratio. The free ends of 2 octrodes were connected to the holes of an EIB-18 (electrode interface board, Neuralynx) and fixed with pins. We designed and manufactured a micro-drive system (home-made 3D conception and printing) consisting of a frame on which is mounted the EIB, and a platform on which are glued the 2 octrodes. Using a driving screw, we were able to slide the platform up and down within the frame, allowing to move through the VTA during chronic recordings in order to sample neuronal populations.

Micro-drive implantation: After anesthetic procedure, the cranial bone of the mouse was exposed by a midline incision of the scalp. The skull was drilled and recording electrodes were placed just above the VTA (bregma - $3.2 \pm 0.1 \mathrm{~mm}$, lateral $0.5 \pm 0.1 \mathrm{~mm}$, ventral $4.1 \pm 0.1 \mathrm{~mm}$ from the brain surface) (Paxinos and Franklin, 2004). A small amount of petroleum jelly (Vaseline) was applied on top the hole and around the recordings electrodes to prevent clotting and facilitate sliding for the following weeks. Monopolar ground electrodes were laid over the cortical layer of the cerebellum, cemented to the skull with SuperBond (Sun Medical) and pinned on the EIB during surgery. SuperBond and dental acrylic cement were then used to fix the micro-drive to the skull for chronic recordings. The scalp was stitched and buprenorphine was injected subcutaneously to facilitate awakening. Animals recovered until regaining pre-surgery body weight, for at least one week.

Neuronal recordings and characterization of DA neurons: Recordings of extracellular potentials were performed using a digital acquisition system (Digital Lynx SX; Neuralynx) together with the Cheetah software. Signals from each wire were band-pass-filtered between 600 and $6000 \mathrm{~Hz}$ for multi-unit recordings at $32 \mathrm{kHz}$ sampling. Spikes sorting and clustering were performed using SpikeSort3D (Neuralynx), and validation of clusters was done with custom-written Python routines based on activity and waveform criteria as well as auto- and cross-correlograms. From the starting position after surgery (around $4.10 \mathrm{~mm}$ ), electrodes were lowered (75 $\mu \mathrm{m}$ steps) every other day to sample as many neurons as possible until a depth of $5.0 \mathrm{~mm}$ was reached. The electrophysiological characteristics of VTA neurons were assessed each time an active cell was encountered. Extracellular identification of putative DA neurons ( $p D A n$ ) was based on their location as well as on a set of unique electrophysiological properties that characterize these cells in vivo: (1) a typical triphasic action potential with a marked negative deflection; (2) a characteristic long duration (>2.0 ms) action potential; (3) an action potential width from start to negative trough $>1.1 \mathrm{~ms}$; (4) a slow firing rate $(1-10 \mathrm{~Hz})$ with a regular single spiking pattern and occasional short bursting activity. Putative GABA neurons were characterized by a characteristic short duration of action potential from start to negative trough $(<1.0 \mathrm{~ms})$, and a high firing rate $(>12 \mathrm{~Hz})$. D2 receptor (D2R) pharmacology was also used to confirm DA neuron identification: after a baseline (10 min) and a saline (5 min) IP injection, $0.2 \mathrm{~mL}$ of quinpirole $(1 \mathrm{mg} / \mathrm{kg}$, D2R agonist) was injected (10 min recording), followed by $0.2 \mathrm{~mL}$-eticlopride (1 mg/kg, D2R antagonist) injection (10 min recording). Since most DA, but not GABA neurons, express inhibitory D2 auto-receptors, neurons were considered as pDAn, if quinpirole induced at least $30 \%$ decrease in their firing rate, while eticlopride restored firing above the baseline. Nevertheless, as continuous D2 pharmacology could have affected DA neurons firing, we allowed mice to recover two days after this experiment and we did not test all encountered pDAn. We

757 thus performed pharmacological confirmation when first encountering a pDA neuron in a given mouse or 
at the end of the week if at least one putative neuron was present. Neurons were considered DA only if they responded to the pharmacology, or if they presented electrophysiological characteristics defined above and were recorded between two positive pharmacological experiments. We assessed the pDAn responses to nicotine injection in the tail vein of the animal (catheter implantation presented above see Fiber photometry section, Figure S2B). We let the animal recover after catheter implantation and habituate to the plastic cylinder for $30 \mathrm{~min}$, and then started to record VTA neurons. For each session, we recorded $7 \mathrm{~min}$ of baseline, $7 \mathrm{~min}$ after saline injection, $15 \mathrm{~min}$ after $30 \mu \mathrm{g} / \mathrm{kg}$ nicotine injection and $20 \mathrm{~min}$ after $60 \mu \mathrm{g} / \mathrm{kg}$ nicotine injection. After the session, mice were re-anesthetized to carefully remove the catheter and were allowed to rest for one day before the next recording session. The screw was turned to lower the octrodes into the VTA and try to sample new pDAn within the following days.

\section{Ex vivo patch-clamp recordings}

771 For a functional verification of CatCh or Jaws expression, AAV5.EF1 1 .DIO.CatCh.YFP or AAV5.EF1 $\alpha . D I O . J a w s . e G F P$ virus was injected into 7 to 9-week-old male DATiCRE mice. For the characterization of NAc-projecting and Amg-projecting neurons, green retrobead tracers (Lumafluor) were injected into 7-9 week old male WT mice. After 4 weeks (for DAT-Cre mice) or 2 weeks (for WT mice), mice were deeply anesthetized by an intraperitoneal injection of a mix of ketamine (150 mg/kg Imalgene $\circledR$ 1000 , Merial) and xylazine (60 mg/kg, Rompun® $2 \%$, Bayer). Coronal midbrain sections (250 $\mu \mathrm{m})$ were sliced with a Compresstome (VF-200, Precisionary Instruments) after intracardial perfusion of cold $\left(4^{\circ} \mathrm{C}\right)$ sucrose-based artificial cerebrospinal fluid (SB-aCSF) containing (in $\mathrm{mM}$ ): $125 \mathrm{NaCl}, 2.5 \mathrm{KCl}, 1.25$ $\mathrm{NaH}_{2} \mathrm{PO}_{4}, 5.9 \mathrm{MgCl}_{2}, 26 \mathrm{NaHCO}_{3}, 25$ sucrose, 2.5 glucose, 1 kynurenate (pH 7.2, 325 mOsm). After 10 to 60 minutes at $35^{\circ} \mathrm{C}$ for recovery, slices were transferred into oxygenated artificial cerebrospinal fluid (aCSF) containing (in mM): $125 \mathrm{NaCl}, 2.5 \mathrm{KCl}, 1.25 \mathrm{NaH}_{2} \mathrm{PO}_{4}, 2 \mathrm{CaCl}_{2}, 1 \mathrm{MgCl}_{2}, 26 \mathrm{NaHCO}_{3}, 15$ sucrose, 10 glucose (pH 7.2, $325 \mathrm{mOsm}$ ) at room temperature for the rest of the day, and individually transferred to a recording chamber continuously perfused at $2 \mathrm{~mL} /$ minute with oxygenated aCSF. Patch pipettes (4$8 \mathrm{M} \Omega$ ) were pulled from thin wall borosilicate glass (G150TF-3, Warner Instruments) with a micropipette puller (P-87, Sutter Instruments, Novato, CA) and filled with a potassium gluconate-based intracellular solution containing (in $\mathrm{mM}$ ): $116 \mathrm{~K}$-gluconate, 20 HEPES, $0.5 \mathrm{EGTA}, 6 \mathrm{KCl}, 2 \mathrm{NaCl}, 4 \mathrm{ATP}, 0.3 \mathrm{GTP}$, and biocytin $2 \mathrm{mg} / \mathrm{mL}$ (pH adjusted to 7.2). Neurons were visualized using an upright microscope coupled with a Dodt contrast lens, and illuminated with a white light source (Scientifica). A 460 nm LED (pE-2, Cooled) was used for visualizing GFP-, YFP- or RB-containing cells (using a bandpass filter cube, AHF). Wholecell recordings were performed with a patch-clamp amplifier (Axoclamp 200B, Molecular Devices) connected to a Digidata (1550 LowNoise acquisition system, Molecular Devices). Signals were low-pass filtered (Bessel, $2 \mathrm{kHz}$ ) and collected at $10 \mathrm{kHz}$ using the data acquisition software pClamp 10.5 (Molecular Devices). Optical stimulation was applied through the microscope with two LEDs (460 nm and $525 \mathrm{~nm}, \mathrm{pE}-2$, CoolLED). To characterize CatCh expression, a $1 \mathrm{~s}$ continuous photostimulation was used to evoke currents in voltage-clamp mode $(-60 \mathrm{mV})$, and a $10 \mathrm{~Hz}-5 \mathrm{~ms} /$ pulse photostimulation was used to drive neuronal firing in current-clamp mode. Regarding Jaws expression, continuous photostimulation (20 s) was used in current-clamp (-60 mV). To record nicotinic currents from RB+DA neurons of the VTA, local puffs $(500 \mathrm{~ms})$ of nicotine tartrate $(100 \mu \mathrm{M}$ in aCSF) were applied with a glass pipette $(2-3 \mu \mathrm{m}$ diameter) positioned 20 to $30 \mu \mathrm{m}$ away from the soma and connected to a picospritzer (World Precision

800 Instruments, adjusted to $\sim 2 \mathrm{psi}$ ). All electrophysiological recordings were extracted using Clampfit 801 (Molecular Devices) and analyzed with R. 
803 After euthanasia, brains were rapidly removed and fixed in $4 \%$ paraformaldehyde. After a period of at

804

805

806

807

808

809

810

811

812

813

814

815

816

817

818

819

820

821

822

823

824

825

\section{6}

827

828

829

830

\section{1}

832

833

834

835

836

837

838

839

840 least three days of fixation at $4^{\circ} \mathrm{C}$, serial 60 - $\mu \mathrm{m}$ sections were cut from the midbrain with a vibratome. Immunostaining experiments were performed as follows: free-floating VTA brain sections were incubated for 1 hour at $4^{\circ} \mathrm{C}$ in a blocking solution of phosphate-buffered saline (PBS) containing $3 \%$ bovine serum albumin (BSA, Sigma; A4503) (vol/vol) and 0.2\% Triton X-100 (vol/vol), and then incubated overnight at $4^{\circ} \mathrm{C}$ with a mouse anti-tyrosine hydroxylase antibody (anti-TH, Sigma, T1299) and a chicken anti-GFP antibody (Life technologies Molecular Probes, A-6455), both at 1:500 dilution, in PBS containing 1.5\% BSA and $0.2 \%$ Triton $X-100$. The following day, sections were rinsed with PBS, and then incubated for 3 hours at $22-25^{\circ} \mathrm{C}$ with $\mathrm{Cy3}$-conjugated anti-mouse and Alexa488-conjugated anti-chicken secondary antibodies (Jackson ImmunoResearch, 715-165-150 and 711-225-152) at 1:500 and 1:1000 dilution in a solution of $1.5 \%$ BSA in PBS, respectively. After three rinses in PBS, slices were wet-mounted using Prolong Gold Antifade Reagent (Invitrogen, P36930). Microscopy was carried out with a fluorescent microscope, and images captured using a camera and analyzed with ImageJ.

In the case of electrophysiological recordings, the recorded neurons were identified by immunohistofluorescence as described above, with the addition of 1:200 AMCA-conjugated streptavidin (Jackson ImmunoResearch) in the solution. Immunoreactivity for both $\mathrm{TH}$ and neurobiotin (NB) allowed us to confirm the neurochemical phenotype of DA neurons in the VTA $(\mathrm{TH}+\mathrm{NB}+)$.

In the case of optogenetic experiments on DATICRE mice, identification of the transfected neurons by immunofluorescence was performed as described above, with the addition of chicken-anti-GFP primary antibody (1:500, ab13970, Abcam) in the solution. A goat-anti-chicken AlexaFluor 488 (1:500, Life Technologies) was then used as secondary antibody. Immunoreactivity for TH, GFP and neurobiotin/biocytin allowed us to confirm the neurochemical phenotype of DA neurons in the VTA $(\mathrm{TH}+$ $\mathrm{NB}+$ ) and the transfection success (GFP+).

\section{Image acquisition}

For immunofluorescence pictures, all slices were imaged by acquisition on a Leica DMR epi-fluorescent microscope, under identical conditions of magnification, illumination and exposure (using photometrics coolsnap camera). Images were captured in gray level using MetaView software (Universal Imaging Corporation, Ropper Scientific, France) and colored post-acquisition on ImageJ software.

\section{Elevated 0-maze test}

All behavioral tests were conducted during the light period of the animal cycle (between 1:00 and 7:00PM). The raw data for behavioral experiments were acquired as the time spent by animals in the different zones of the environments. Animals were detected in their body center with a 2D USB camera, connected to the Anymaze software for acquisition.

The elevated O-maze (EOM) apparatus consists of two open (stressful) and two enclosed (protecting) elevated arms that together form a zero or circle (diameter of $50 \mathrm{~cm}$, height of $58 \mathrm{~cm}, 10 \mathrm{~cm}$-wide circular platform). The time spent in exploring enclosed versus open arms indicates the anxiety level of the animal. The first EOM experiment assessed the effect of an IP injection of Nic $(0.5 \mathrm{mg} / \mathrm{kg})$ on WT mice. The test lasts 10 minutes: mice are injected 1 minute before the test, and then put in the EOM for 9 minutes. In 
841 the second EOM experiment, mice received an IC infusion of Nic ( $100 \mathrm{ng} /$ infusion) over 1 minute before 842 the 9-minute test. Finally, optogenetic EOM experiments consisted in 15 minute-test, alternating 5 minute843 periods of stimulation and non-stimulation (OFF-ON-OFF). For "rescue" experiment in EOM, nicotine was 844 injected IP to the mice with the same protocol as described above, the test lasted for 9 minutes with continuous stimulation along the test (continuous for inhibition and $10 \mathrm{~Hz}$ for activation).

\section{Real-time place preference test}

847 The real-time place preference (RTPP) protocols were performed in a Y-maze apparatus (Imetronic, 848 Pessac, France), using only two arms of the Y-maze as two distinct compartments (the third arm was 849 closed by a door and not available to the animal). The chamber in between is an equilateral triangle (side 850 of $11 \mathrm{~cm}$ ) used as a neutral compartment, where the animal was never photo-stimulated. Each arm of the 851 maze measures $25 \mathrm{~cm} \times 12 \mathrm{~cm}$. The first arm displays black and white stripes with smooth walls and 852 floor, whereas the other arm displays uniform-gray rough walls and floor. Choices of the compartment 853 where the animals will be stimulated were counterbalanced across animals in the same test and YFP854 control groups.

855 The RTPP test consisted of a 20 minute-session where animals can freely navigate between the 856 compartments but are photo-stimulated only in one of the two compartments.

857 Implanted animals were connected with a bilateral fiber (diameter of $400 \mu \mathrm{m}, \mathrm{NA}=0.39$, Thorlabs) 858 attached to a rotor connecting the $470 \mathrm{~nm}$-LED or $520 \mathrm{~nm}$-LED (Prizmatix) with a fiber of diameter 500 $859 \mu \mathrm{m}$ and $\mathrm{NA}=0.5$ (Thorlabs). LED output was controlled using a Master-8 pulse stimulator (A.M.P.I., 860 Jerus) which delivered a discontinuous stimulation of 5-ms light flashes at $10 \mathrm{~Hz}$ frequency and $470 \mathrm{~nm}$ 861 wavelength (for CatCh experiments), or a continuous stimulation at $520 \mathrm{~nm}$ (for Jaws experiments). Naive 862 mice were connected and placed at the center of the neutral compartment before starting the recording. 863 The time spent in the neutral compartment was not taken into account in the result. The results are 864 presented as preference score which is the difference of time spent between the stimulated compartment 865 over the "non-stimulated" compartment.

866

867

868

869

870

871

872

873

874

875

876

877

878

879

880

\section{Open field paradigm}

The open field (OF) is a square enclosure of $50 \mathrm{~cm} \times 50 \mathrm{~cm}$ where animals can move freely. Animal displacements were quantified by comparing the time spent in the center versus the periphery of the square. When nicotine was injected to WT mice in the OF test (IP injection of nicotine tartrate at $0.5 \mathrm{mg} / \mathrm{kg}$, $0.1 \mathrm{~mL} / 10 \mathrm{~g}, 1$ minute before the test), animals were placed in the center of the $\mathrm{OF}$ for a 9-minute test duration, freely moving inside the enclosure. Regarding the optogenetic experiments conducted in the OF, animals were placed in the maze for 15 minutes, while alternating between OFF, ON and OFF optical stimulations periods of 5 minutes each.

\section{QUANTIFICATION AND STATISTICAL ANALYSIS}

\section{Measurements of neuronal activity}

Timestamps of action potentials were extracted in Spike 2 and analyzed using $R$, a language and environment for statistical computing (Team, 2005, http://www.r-project.org). Spontaneous activity of DA cell firing in vivo was analyzed with respect to the average firing frequency (in $\mathrm{Hz}$ ) and the percentage of spikes-within-burst (\%SWB = number of spikes within burst divided by total number of spikes in a given window). Neuronal basal activity was defined on at least three-minute recording. To determine whether 
881

882

883

884

885

886

887

888

889

890

891

892

893

894

895

896

897

898

899

900

901

902

903

904

905

906

907

908

909

910

911

912

913

914

915

916

917

918

919

920

921

922

the spontaneous activity of VTA DA neurons could predict their responses to nicotine injection (activation or inhibition), we analyzed 4 variables that characterize the firing patterns: the mean firing frequency, the coefficient of variation of the firing frequency estimated on sliding windows, the \%SWB and the burs event frequency. For multiple logistic regression, glm function ( $R$ 4.0, with binomial family) was used for fitting and predict.glm function wad used to obtain prediction.

\section{Method for classifying VTA DA neurons subpopulations in response to nicotine injection}

Subpopulations of DA neurons were automatically classified using variation of firing frequency and the following routine: First, we calculated the maximal variation from the baseline per neuron, within the first 3 (for anesthetized animals) or 5 minutes (for freely-moving mice) following injection. We then used a bootstrapping method (see below) to exclude non-responding neurons. Two neurons included in Fig $1 \mathrm{~B}$ $(n=245)$ did not show statistical variations after nicotine injections and were thus removed from the rest of the study. Neurons displaying an increase in firing frequency $(\Delta f>0)$ were defined as "Nic+", while neurons displaying a decrease in firing frequency $(\Delta \mathrm{f}<0)$ were defined as "Nic-". For the dose-response curve, neurons were classified as Nic+ or Nic- based on their response to a nicotine dose of $30 \mu \mathrm{g} / \mathrm{kg}$ or higher. For saline injections, only nicotine-responsive neurons were considered, and the polarity of the variation was defined based on the response to nicotine (i.e. in Nic+ neurons, we consider that saline increases activity). In $\beta 2-$ mice, VTA DA neurons did not show a clear change in firing rate after nicotine injection. In $\beta 2 \mathrm{Vec}$ mice, lentivirus-mediated expression of $\beta 2$ is most likely heterogenous within the VTA, hence not all recorded cells are expected to respond to nicotine. In $\beta 2^{--}$and $\beta 2 \mathrm{Vec}$ mice, we thus divided populations of neurons using the threshold criteria $(\Delta f<0$ or $>0)$ and evaluated the impact of re-expression without using the bootstrapping method (Figure 3B and S8A-D). Responding neurons (boostrapping at $2 \%$ ) are shown in Figure S8E-F. Overall, for $\beta 2^{--}$mice, only $22 / 46$ neurons $(47 \%)$ showed a response (bootstrapping at $2 \%$ ), while for $\beta 2 v e c$ mice this ratio increased to $65 / 90(72 \%)$.

\section{Quantification of neuronal responses to nicotine injection}

Firing frequency was quantified on overlapping 60 -second windows shifted by 15 -second time steps (except for Figure $\mathrm{S1}$, in which windows are shifted by $1 \mathrm{sec}$ steps). For each neuron, the firing frequency was rescaled as a percentage of its baseline value averaged during 3 minutes before nicotine injection. The responses to nicotine are thus presented as a percentage of variation from baseline (mean \pm S.E.M.). The effect of nicotine was assessed by comparing the maximum of firing frequency variation induced by nicotine and saline injection. For activated (respectively inhibited) neurons, the maximal (respectively minimal) value of firing frequency was measured within the response period ( 3 to 5 minutes) that followed nicotine or saline injection. The results are presented as mean \pm S.E.M. of the difference of maximum variation after nicotine or saline. The mean responses to nicotine injections for recordings in freely moving mice, both for inhibited and activated groups, pooled 7 responses at $30 \mu \mathrm{g} / \mathrm{kg}$ Nic and one response at $60 \mu \mathrm{g} / \mathrm{kg}$. Neurons that significantly responded to nicotine injections were identified by bootstrapping. Baseline spike intervals were randomly shuffled 1000 times. Firing frequency was estimated on $60 \mathrm{sec}$ time windows, with $15 \mathrm{sec}$ time steps. For each neuron we determined the percentile from the shuffled data corresponding to the nicotine-evoked response (max or min frequency after nicotine injection). Neurons were individually considered as responsive to nicotine injection if this percentile is $\geq 0.98$ or $\leq 0.02$.

\section{Quantification of juxtacellularly labeled neurons}


923 A total number of 245 neurons were recorded and labeled for Figure 1. Those 245 neurons were used in 924 Figure 1B. Two non-responding neurons were removed, so 243 neurons were used for Fig1C-D and G. 925 Among them, 101 neurons were shown in Figure 2B and E, with 49 neurons labeled in NAc-RB injected 926 mice and 52 in Amg-RB injected mice. The locations of the labeled neurons were manually placed on 927 sections of the Paxinos atlas georeferenced in a 2D grid using Adobe Illustrator rules. The medio-lateral 928 and dorso-ventral coordinates of the location of each neuron were extracted from the grid pattern and the 929 antero-posterior coordinates were estimated from the section of the Paxinos atlas on which the neurons 930 were placed. These three coordinates were used to make density histograms of location for nicotine931 activated and nicotine-inhibited DA neurons or NAc-projecting and Amg-projecting DA neurons.

\section{Quantification of fluorescence}

933 Data from fiber photometry experiments were first down-sampled by a 100-factor using custom Matlab 934 routine. Down-sampled data were then further analyzed on $\mathrm{R}$ software. First, we subtracted the mean 935 value of "autofluorescence" (signal acquired after each recording with the same parameters, but without 936 the optic fiber attached to the mouse) to the signal. We then fitted an exponential to this signal and 937 subtracted it before adding an offset equal to the mean of the signal before detrending to account for the 938 slow decay of the signal due to bleaching during recording. We defined a baseline fluorescence value

939 (F0) as the mean fluorescence of the signal during 120 seconds before injection time, for each injection 940 (saline and nicotine) individually. We then calculated normalized variation in fluorescence $(\Delta F / F)$ as $(F$ 941 F0)/F0 for each injection. The analysis was carried out by averaging each $\Delta F / F$ obtained for each 942 condition (all saline or nicotine injections done in NAcLSh implanted mice, same for saline or nicotine in 943 BLA animals) and mean data were smoothed using a normal kernel.fit (bandwidth $=120$ ). All Nic 944 responses $(n=7)$ for BLA implantation were done at $30 \mu \mathrm{g} / \mathrm{kg}$ recorded in 6 different animals. For NAcLSh implantation, 6 animals received Nic injection at 30 or $60 \mu \mathrm{g} / \mathrm{kg}(\mathrm{n}=8$, mean fluorescence pooled 2 injections at $60 \mu \mathrm{g} / \mathrm{kg}$ and 6 injections at $30 \mu \mathrm{g} / \mathrm{kg}$ ). For each injection (saline and nicotine), peak fluorescence (maximum and minimum of $\triangle F / F$ for NAcLSh and BLA implanted mice respectively) were detected within a $100 \mathrm{sec}$ window after injection. For each paired injection, we calculated the difference in peak fluorescence between nicotine and saline.

\section{Statistics: figure by figure}

951 All statistical analyses were done using the R software with home-made routines. Results are plotted as 952 mean \pm S.E.M. The total number $(n)$ of observations in each group and the statistical tests used for 953 comparisons between groups or within groups are indicated on the figures directly or in the figure legends.

954 Comparisons between means were performed with parametric tests as Student's t-test, or two-way 955 ANOVA for comparing two groups when parameters followed a normal distribution (Shapiro-Wilk 956 normality test with $p>0.05$ ), or Wilcoxon non-parametric test as when the distribution was skewed. Holm's 957 sequential Bonferroni post hoc analysis was applied, when necessary. Statistical significance was set at $958 p<0.05\left(^{*}\right), p<0.01\left(^{* *}\right)$, or $p<0.001\left(^{* * *}\right)$, or $p>0.05$ was considered not to be statistically significant.

959 Figure 1: Kolmogorov-Smirnov test was used to compare the responses of VTA DA neurons to saline or 960 nicotine injection. Wilcoxon test was used to demonstrate a significant increase or decrease of firing 961 frequency induced by nicotine injection compared to saline injection (B). Wilcoxon test was used to 962 compare the firing frequency before and after nicotine or saline injection. Wilcoxon test was used to 963 compare the firing frequency variation induced by nicotine or saline injection. (D). One-way ANOVA was 
964

965

966

967

968

969

970

971

972

973

974

975

976

977

978

979

980

981

982

983

984

985

986

987

988

989

990

991

992

993

994

995

996

997

998

999

1000

used to demonstrate a dose-effect of nicotine on activated or inhibited DA neurons (E). Wilcoxon test was used to compare coordinates of nicotine-inhibited and nicotine-activated recorded neurons $(G)$.

Figure 2: Wilcoxon test was used to compare the firing frequency before and after nicotine in $\mathrm{RB}+$ and RB- DA neurons. Wilcoxon test was used to compare firing frequency variations induced by nicotine in $\mathrm{RB}+$ and RB- DA neurons (C-F). Kolmogorov-Smirnov test was used to compare the responses of NAcprojecting and Amg-projecting DA neurons to nicotine injection $(G)$. Paired Student's t-test was used to compare the peak of fluorescence induced by saline and nicotine injection in NAcLSh or BLA. Student's $\mathrm{t}$-test was used to compare the difference in $\triangle F / F$ (Nicotine - Saline) between NAcLSh and BLA (I).

Figure 3: For behavior (A-C), over time effect of nicotine or saline injection (IP and IC) on the time spent by the mice in the open arms of the EOM was first tested with one-way repeated measures ANOVA for each group of mice (shown in Figure S7A-D-G). Two-way repeated measures ANOVA (time/treatment or time/genotype) were used to compare the difference between the groups. In case of significant interaction effect between factors, Wilcoxon or Student's t-test with Holm's sequential Bonferroni corrections were used for intra-group and inter-group post hoc analysis (as indicated in the figure). For electrophysiology (B), Kolmogorov-Smirnov test was used to compare responses to nicotine of DA neurons in $\beta 2^{-- \text {mice }}$ and $\beta 2--$ Vec mice. Wilcoxon tests with Bonferroni corrections are used to demonstrate a significant increase or decrease of firing frequency induced by IV nicotine injection in $\beta 2^{-/-V e c}$ mice compared to saline and nicotine injections in $\beta 2^{--}$mice.

Figure 4 and 5: For EOM experiments (A-B), effect of light was first tested with one-way repeated measures ANOVA for each group of mice (shown in Figure S11A-B and Figure S13A-B). Two-way repeated measures ANOVA (time/opsin) were used to compare the difference between the groups. In case of a significant interaction effect between factors, Wilcoxon or Student's t-test with Holm's sequential Bonferroni corrections were used for intra-group and inter-group post hoc analysis (as indicated in the figure). For EOM experiment under nicotine (C), over time effect of nicotine injection on the time spent by the mice in the EOM open arms was first tested with one-way repeated measures ANOVA for each group. Two-way repeated measures ANOVA (time/opsin) were used to compare the difference between the groups. In case of a significant interaction effect between factors, Wilcoxon or Student's t-test with Bonferroni corrections were used for intra-group and inter-group post hoc analysis (as indicated in the figure). For RTPP experiments (D), preference scores between groups were compared with Student's ttest.

Figure S2: Wilcoxon test was used to compare spontaneous activity of nicotine-inhibited and nicotineactivated recorded neurons $(\mathrm{C})$.

Figure S4: Wilcoxon test was used to compare coordinates of NAc- and Amg-projecting neurons (E). Wilcoxon test was used to compare spontaneous activity of NAc- and Amg- projecting DA neurons (F).

Figure S5: Wilcoxon test was used to compare firing frequencies before and after nicotine in $\mathrm{RB}+$ and RB- DA neurons. Wilcoxon test was used to compare firing frequency variations induced by nicotine in $\mathrm{RB}+$ and RB- DA neurons $(\mathrm{D} / \mathrm{H})$. 
1001 Figure S6: Two-way repeated measures ANOVA (current, phenotype) was used to compare neuronal excitability (D). Wilcoxon test was used to compare nicotine-evoked currents (F).

1003 Figure S7: Two-way repeated measures ANOVA (time, treatment) was used to compare the distance

1004

1005

1006

1007

1008

1009

1010

1011

1012

1013

1014

1015

1016

1017

1018

1019

1020

1021

1022

1023

1024

1025

1026

1027

1028

1029

1030 traveled by mice in the OF after nicotine or saline injection (A). One-way repeated measures ANOVA were used to test the overtime effect of saline or nicotine intraperitoneal injection, or intracranial infusion, or the time spent by mice in the open arms of the EOM. Two-way repeated measures ANOVA (time, treatment) was used to compare number of entries in the open arms of the EOM after saline or nicotine injection (A, D, G). Two-way repeated measures ANOVA (time, genotype) was used to compare the time spent and the number of entries in the EOM open arms after nicotine injection between groups (A-D-F$\mathrm{G})$. In case of a significant interaction effect between factors, Wilcoxon or Student's t-test with Holm's sequential Bonferroni corrections were used for intra-group and inter-group post hoc analysis.

Figure S8: Kolmogorov-Smirnov test was used to compare the responses of VTA DA neurons to saline or nicotine injection in $\beta 2^{--}(A)$ and $\beta 2^{--}-\operatorname{Vec}$ mice $(C)$. Wilcoxon test was used to compare firing frequency before and after nicotine or saline injection. Wilcoxon test was used to compare firing frequency variation induced by nicotine or saline injection in $\beta 2^{-/-}(B-G-F)$ and $\beta 2^{--}$Vec mice (D-G-F).

Figure S11and 13: For anxiety measurements, one-way repeated measures ANOVA were used to test the light effect on the time spent in the open arms of the EOM. Two-way repeated measures ANOVA were used to compare the stimulation effect depending on the position of the animal (closed arms or open arms) at the onset of the light and the number of entries in the open arms of the EOM between groups (A-B). For locomotor activity, two-way repeated measures ANOVA (time, opsin) were used to compare the difference of light effect on the distance traveled by the mice between the groups $(C)$. In case of a significant interaction effect between factors, Wilcoxon or Student's t-test with Holm's sequential Bonferroni corrections were used for intra-group and inter-group post hoc analysis (as indicated in the figure).

Figure S12: EOM experiments (C-D) and locomotor activity (E-F) were analyzed as previously described for Figure S9.

Figure S14: For EOM experiments, the time spent in the open arms (cf. Figure 3), and the number of entries were analyzed as previously described (cf. Figure S11). Locomotor activity was analyzed as previously described in Figure S11. For RTPP experiments (E), preference scores between groups were compared with Student's t-test. 


\section{References}

1032 Aurnhammer, C., Haase, M., Muether, N., Hausl, M., Rauschhuber, C., Huber, I., Nitschko, H., Busch, 1033 U., Sing, A., Ehrhardt, A., et al. (2012). Universal real-time PCR for the detection and quantification of 1034 adeno-associated virus serotype 2-derived inverted terminal repeat sequences. Hum Gene Ther 1035 Methods 23, 18-28.

1036 Balerio, G.N., Aso, E., and Maldonado, R. (2006). Role of the cannabinoid system in the effects induced by nicotine on anxiety-like behaviour in mice. Psychopharmacology 184, 504-513.

1038 Beier, K.T., Gao, X.J., Xie, S., DeLoach, K.E., Malenka, R.C., and Luo, L. (2019). Topological

1039 Organization of Ventral Tegmental Area Connectivity Revealed by Viral-Genetic Dissection of Input-

1040 Output Relations. CellReports 26, 159-167.e6.

1041 Beier, K.T., Steinberg, E.E., DeLoach, K.E., Xie, S., Miyamichi, K., Schwarz, L., Gao, X.J., Kremer, E.J., 1042 Malenka, R.C., and Luo, L. (2015). Circuit Architecture of VTA Dopamine Neurons Revealed by 1043 Systematic Input-Output Mapping. Cell 162, 622-634.

1044 Brischoux, F., Chakraborty, S., Brierley, D.I., and Ungless, M.A. (2009). Phasic excitation of dopamine neurons in ventral VTA by noxious stimuli. Proc Natl Acad Sci USA 106, 4894-4899.

Changeux, J.-P., Bertrand, D., Corringer, P.J., Dehaene, S., Edelstein, S., Léna, C., Le Novère, N., Marubio, L., Picciotto, M., and Zoli, M. (1998). Brain nicotinic receptors: structure and regulation, role in learning and reinforcement. Brain Res Brain Res Rev 26, 198-216.

1049 Changeux, J.-P. (2010). Nicotine addiction and nicotinic receptors: lessons from genetically modified mice. Nat Rev Neurosci 11, 389-401.

1051 Choi, V.W., Asokan, A., Haberman, R.A., and Samulski, R.J. (2007). Production of recombinant adeno-

Chuong, A.S., Miri, M.L., Busskamp, V., Matthews, G.A.C., Acker, L.C., Sørensen, A.T., Young, A., Klapoetke, N.C., Henninger, M.A., Kodandaramaiah, S.B., et al. (2014). Noninvasive optical inhibition with a red-shifted microbial rhodopsin. (Nature Publishing Group).

1056 de Jong, J.W., Afjei, S.A., Pollak Dorocic, I., Peck, J.R., Liu, C., Kim, C.K., Tian, L., Deisseroth, K., and Lammel, S. (2019). A Neural Circuit Mechanism for Encoding Aversive Stimuli in the Mesolimbic Dopamine System. Neuron 101, 133-151.e137.

1059 Di Chiara, G., and Imperato, A. (1988). Drugs abused by humans preferentially increase synaptic 1060 dopamine concentrations in the mesolimbic system of freely moving rats. Proc Natl Acad Sci USA 85, 1061 5274-5278. Decreases Ethanol-Induced Dopamine Signaling and Increases Self-Administrationvia Stress

1064 Hormones. Neuron 1-11.

1065 Durand-de Cuttoli, R., Mondoloni, S., Marti, F., Lemoine, D., Nguyen, C., Naudé, J., d'Izarny-Gargas, T., 1066 Pons, S., Maskos, U., Trauner, D., et al. (2018). Manipulating midbrain dopamine neurons and rewardrelated behaviors with light-controllable nicotinic acetylcholine receptors. eLife 7, 15991.

1068 Eddine, R., Valverde, S., Tolu, S., Dautan, D., Hay, A., Morel, C., Cui, Y., Lambolez, B., Venance, L., Marti, F., et al. (2015). A concurrent excitation and inhibition of dopaminergic subpopulations in response to nicotine. Sci. Rep. 5, 8184.

1071 Ford, C.P., Mark, G.P., and Williams, J.T. (2006). Properties and opioid inhibition of mesolimbic 1072 dopamine neurons vary according to target location. Journal of Neuroscience 26, 2788-2797. 
1073 Fowler, C.D., Lu, Q., Johnson, P.M., Marks, M.J., and Kenny, P.J. (2011). Habenular a5 nicotinic

1074 receptor subunit signalling controls nicotine intake. Nature 471, 597-601.

1075 Frahm, S., Ślimak, M.A., Ferrarese, L., Santos-Torres, J., Antolin-Fontes, B., Auer, S., Filkin, S., Pons, 1076 S., Fontaine, J.-F., Tsetlin, V., et al. (2011). Aversion to Nicotine Is Regulated by the Balanced Activity 1077 of \&beta;4 and \&alpha;5 Nicotinic Receptor Subunits in the Medial Habenula. Neuron 70, 522-535.

1078 Grace, A.A., and Bunney, B.S. (1984a). The control of firing pattern in nigral dopamine neurons: burst 1079 firing. J Neurosci 4, 2877-2890.

1080 Grace, A.A., and Bunney, B.S. (1984b). The control of firing pattern in nigral dopamine neurons: single 1081 spike firing. J Neurosci 4, 2866-2876.

1082 Grenhoff, J., Aston-Jones, G., and Svensson, T.H. (1986). Nicotinic effects on the firing pattern of 1083 midbrain dopamine neurons. Acta Physiol. Scand. 128, 351-358.

1084 Grieder, T.E., George, O., and Tan, H. (2012). Phasic D1 and tonic D2 dopamine receptor signaling 1085 double dissociate the motivational effects of acute nicotine and chronic nicotine withdrawal.

1086 Grieder, T.E., Besson, M., Maal-Bared, G., Pons, S., Maskos, U., and van der Kooy, D. (2019). ß2* nAChRs on VTA dopamine and GABA neurons separately mediate nicotine aversion and reward. Proc 1088 Natl Acad Sci USA 116, 25968-25973.

1089 Grieder, T.E., Sellings, L.H., Vargas-Perez, H., Ting-A-Kee, R., Siu, E.C., Tyndale, R.F., and van der 1090 Kooy, D. (2010). Dopaminergic signaling mediates the motivational response underlying the opponent 1091 process to chronic but not acute nicotine. Neuropsychopharmacology 35, 943-954.

1092 Khabou, H., Garita-Hernandez, M., Chaffiol, A., Reichman, S., Jaillard, C., Brazhnikova, E., Bertin, S., 1093 Forster, V., Desrosiers, M., Winckler, C., et al. (2018). Noninvasive gene delivery to foveal cones for 1094 vision restoration. JCI Insight 3, D358.

1095 Kleinlogel, S., Feldbauer, K., Dempski, R.E., Fotis, H., Wood, P.G., Bamann, C., and Bamberg, E. 1096 (2011). Ultra light-sensitive and fast neuronal activation with the $\mathrm{Ca}^{2}+$-permeable channelrhodopsin 1097 CatCh. 14, 513-518.

1098 Klink, R., de Kerchove d'Exaerde, A., Zoli, M., and Changeux, J.P. (2001). Molecular and physiological 1099 diversity of nicotinic acetylcholine receptors in the midbrain dopaminergic nuclei. J Neurosci 21, 145211001463.

1101 Kutlu, M.G., and Gould, T.J. (2015). Nicotine Modulation of Fear Memories and Anxiety: Implications for 1102 Learning and Anxiety Disorders. Biochem Pharmacol 1-58.

1103 Lammel, S., Hetzel, A., Häckel, O., Jones, I., Liss, B., and Roeper, J. (2008). Unique properties of 1104 mesoprefrontal neurons within a dual mesocorticolimbic dopamine system. Neuron 57, 760-773.

1108 Luscher, C. (2016). The Emergence of a Circuit Model for Addiction. Annu Rev Neurosci 39, annurev1109 neuro-070815-013920.

1110 Mameli-Engvall, M., Evrard, A., Pons, S., Maskos, U., Svensson, T.H., Changeux, J.-P., and Faure, P. 1111 (2006). Hierarchical control of dopamine neuron-firing patterns by nicotinic receptors. Neuron 50, 9111112921.

1113 Mansvelder, H.D., and McGehee, D.S. (2000). Long-term potentiation of excitatory inputs to brain 1114 reward areas by nicotine. Neuron 27, 349-357. 
Margolis, E.B., Hjelmstad, G.O., Fujita, W., and Fields, H.L. (2014). Direct bidirectional $\mu$-opioid control of midbrain dopamine neurons. Journal of Neuroscience 34, 14707-14716.

1117 Margolis, E.B., Mitchell, J.M., Ishikawa, J., Hjelmstad, G.O., and Fields, H.L. (2008). Midbrain dopamine neurons: projection target determines action potential duration and dopamine $\mathrm{D}(2)$ receptor inhibition. Journal of Neuroscience 28, 8908-8913.

Maskos, U., Molles, B.E., Pons, S., Besson, M., Guiard, B.P., Guilloux, J.-P., Evrard, A., Cazala, P., Cormier, A., Mameli-Engvall, M., et al. (2005). Nicotine reinforcement and cognition restored by targeted expression of nicotinic receptors. Nature 436, 103-107.

Mejias-Aponte, C.A., Ye, C., Bonci, A., Kiyatkin, E.A., and Morales, M. (2015). A Subpopulation of Neurochemically-Identified Ventral Tegmental Area Dopamine Neurons Is Excited by Intravenous Cocaine. J Neurosci 35, 1965-1978.

1126 Mineur, Y.S., Fote, G.M., Blakeman, S., Cahuzac, E.L.M., Newbold, S.A., and Picciotto, M.R. (2016). Multiple Nicotinic Acetylcholine Receptor Subtypes in the Mouse Amygdala Regulate Affective Behaviors and Response to Social Stress. Neuropsychopharmacology 41, 1579-1587.

Molas, S., DeGroot, S.R., Zhao-Shea, R., and Tapper, A.R. (2017). Anxiety and Nicotine Dependence: Emerging Role of the Habenulo-Interpeduncular Axis. Trends Pharmacol Sci 38, 169-180.

Morales, M., and Margolis, E.B. (2017). Ventral tegmental area: cellular heterogeneity, connectivity and behaviour. Nat Rev Neurosci 18, 73-85.

Morel, C., Fattore, L., Pons, S., Hay, Y.A., Marti, F., Lambolez, B., De Biasi, M., Lathrop, M., Fratta, W., Maskos, U., et al. (2014). Nicotine consumption is regulated by a human polymorphism in dopamine neurons. Mol Psychiatry 19, 930-936.

Morel, C., Fernandez, S.P., Pantouli, F., Meye, F.J., Marti, F., Tolu, S., Parnaudeau, S., Marie, H., Tronche, F., Maskos, U., et al. (2017). Nicotinic receptors mediate stress-nicotine detrimental interplay via dopamine cells' activity. Mol Psychiatry 23, 1597-1605.

Pascoli, V., Terrier, J., Hiver, A., and Lüscher, C. (2015). Sufficiency of Mesolimbic Dopamine Neuron Stimulation for the Progression to Addiction. Neuron 88, 1054-1066.

Paxinos, G., and Franklin, K.B.J. (2004). The Mouse Brain in Stereotaxic Coordinates (Gulf Professional Publishing). J.P. (1998). Acetylcholine receptors containing the beta2 subunit are involved in the reinforcing properties of nicotine. Nature 391, 173-177.

1146 Picciotto, M.R., and Mineur, Y.S. (2013). Molecules and circuits involved in nicotine addiction: The many faces of smoking. Neuropharmacology.

Pinault, D. (1996). A novel single-cell staining procedure performed in vivo under electrophysiological control: morpho-functional features of juxtacellularly labeled thalamic cells and other central neurons with biocytin or Neurobiotin. J. Neurosci. Methods 65, 113-136.

1151 Poulin, J.-F., Caronia, G., Hofer, C., Cui, Q., Helm, B., Ramakrishnan, C., Chan, C.S., Dombeck, D.A.,

1152 Deisseroth, K., and Awatramani, R. (2018). Mapping projections of molecularly defined dopamine

1153 neuron subtypes using intersectional genetic approaches. 21,1-17.

1154 Radke, A.K., and Gewirtz, J.C. (2012). Increased dopamine receptor activity in the nucleus accumbens 1155 shell ameliorates anxiety during drug withdrawal. Neuropsychopharmacology 37, 2405-2415.

1156 Schultz, W. (2007). Multiple dopamine functions at different time courses. Annu Rev Neurosci 30, 2591157288. 
1158 Su, M., Li, L., Wang, J., Sun, H., Zhang, L., Zhao, C., Xie, Y., Gamper, N., Du, X., and Zhang, H.

1159 (2019). Kv7.4 Channel Contribute to Projection-Specific Auto-Inhibition of Dopamine Neurons in the

1160 Ventral Tegmental Area. Front Cell Neurosci 13, 557.

1161 Sun, F., Zeng, J., Jing, M., Zhou, J., Feng, J., Owen, S.F., Luo, Y., Li, F., Wang, H., Yamaguchi, T., et

1162 al. (2018). A Genetically Encoded Fluorescent Sensor Enables Rapid and Specific Detection of

1163 Dopamine in Flies, Fish, and Mice. Cell 174, 481-496.e19.

1164 Sun, F., Zhou, J., Dai, B., Qian, T., Zeng, J., Li, X., Zhuo, Y., Zhang, Y., Tan, K., Feng, J., et al. (2020). New and improved GRAB fluorescent sensors for monitoring dopaminergic activity \&lt;em\&gt;in 1166 vivo\&lt;/em\&gt. bioRxiv 2020.03.28.013722.

1167 Tolu, S., Eddine, R., Marti, F., David, V., Graupner, M., Pons, S., Baudonnat, M., Husson, M., Besson, M., Reperant, C., et al. (2013). Co-activation of VTA DA and GABA neurons mediates nicotine reinforcement. Mol Psychiatry 18, 382-393.

1170 Turiault, M., Parnaudeau, S., Milet, A., Parlato, R., Rouzeau, J.-D., Lazar, M., and Tronche, F. (2007). Analysis of dopamine transporter gene expression pattern -- generation of DAT-iCre transgenic mice.

1172 Febs J. 274, 3568-3577.

1173 Ungless, M.A., and Grace, A.A. (2012). Are you or aren't you? Challenges associated with 1174 physiologically identifying dopamine neurons. Tins 35, 422-430.

1175 Watabe-Uchida, M., Zhu, L., Ogawa, S.K., Vamanrao, A., and Uchida, N. (2012). Whole-Brain Mapping 1176 of Direct Inputs to Midbrain Dopamine Neurons. Neuron 74, 858-873.

1177 Wolfman, S.L., Gill, D.F., Bogdanic, F., Long, K., Al-Hasani, R., McCall, J.G., Bruchas, M.R., and

1178 McGehee, D.S. (2018). Nicotine aversion is mediated by GABAergic interpeduncular nucleus inputs to 1179 laterodorsal tegmentum. Nature Communications 9, 2710.

1180 Yang, H., de Jong, J.W., Tak, Y., Peck, J., Bateup, H.S., and Lammel, S. (2018). Nucleus Accumbens

1181 Subnuclei Regulate Motivated Behavior via Direct Inhibition and Disinhibition of VTA Dopamine

1182 Subpopulations. Neuron 97, 434-449.e434.

1183 Zarrindast, M.R., Khalifeh, S., Rezayof, A., Rostami, P., Aghamohammadi Sereshki, A., and

1184 Zahmatkesh, M. (2012). Involvement of rat dopaminergic system of nucleus accumbens in nicotine-

1185 induced anxiogenic-like behaviors. Brain Res 1460, 25-32.

1186 Zhao-Shea, R., Liu, L., Pang, X., Gardner, P.D., and Tapper, A.R. (2013). Activation of GABAergic neurons in the interpeduncular nucleus triggers physical nicotine withdrawal symptoms. Curr Biol 23, 2327-2335.

1189 Zhao-Shea, R., Liu, L., Soll, L.G., Improgo, M.R., Meyers, E.E., Mclntosh, J.M., Grady, S.R., Marks, 1190 M.J., Gardner, P.D., and Tapper, A.R. (2011). Nicotine-mediated activation of dopaminergic neurons in 1191 distinct regions of the ventral tegmental area. Neuropsychopharmacology 36, 1021-1032.

1192 Zhuang, X., Masson, J., Gingrich, J.A., Rayport, S., and Hen, R. (2005). Targeted gene expression in 1193 dopamine and serotonin neurons of the mouse brain. J. Neurosci. Methods 143, 27-32. 


\section{KEY RESOURCES TABLE}

\begin{tabular}{|c|c|c|}
\hline REAGENT or RESOURCE & SOURCE & IDENTIFIER \\
\hline \multicolumn{3}{|l|}{ Antibodies } \\
\hline Anti-tyrosine Hydroxylase produced in mouse & Sigma-Aldrich & $\begin{array}{l}\text { Cat\# T1299, } \\
\text { RRID:AB_477560 }\end{array}$ \\
\hline Anti-GFP produced in chicken & Aveslabs & $\begin{array}{l}\text { Cat\# GFP-1020, } \\
\text { RRID:AB_1000024 } \\
0\end{array}$ \\
\hline Anti-rabbit Cy2-conjugated produced in donkey & Jackson ImmunoResearch & $\begin{array}{l}\text { Cat\# 711-225-152, } \\
\text { RRID:AB_2340612 }\end{array}$ \\
\hline Anti-mouse Cy3-conjugated produced in donkey & Jackson ImmunoResearch & $\begin{array}{l}\text { Cat\# 715-165-150, } \\
\text { RRID:AB_2340813 }\end{array}$ \\
\hline Anti-chicken Alexa488-conjugated & Jackson ImmunoResearch & $\begin{array}{l}\text { Cat\# 703-545-155, } \\
\text { RRID:AB_2340375 }\end{array}$ \\
\hline AMCA-Streptavidin & Jackson ImmunoResearch & $\begin{array}{l}\text { Cat\# 016-150-084, } \\
\text { RRID:AB_2337243 }\end{array}$ \\
\hline \multicolumn{3}{|l|}{ Bacterial and virus strains } \\
\hline Lenti-pGK-B2-IRES-GFP & $\begin{array}{l}\text { Maskos et al., } 2005 \\
\text { https://doi.org/10.1038/nat } \\
\text { ure03694 : } \\
\text { Provided by Institut } \\
\text { Pasteur, Paris, France }\end{array}$ & Virus (Lentivirus) \\
\hline Lenti-pGK-IRES-GFP & $\begin{array}{l}\text { Maskos et al., } 2005 \\
\text { https://doi.org/10.1038/nat } \\
\text { ure03694: } \\
\text { Provided by Institut } \\
\text { Pasteur, Paris, France }\end{array}$ & Virus (Lentivirus) \\
\hline pAAV.Ef1a.DIO.CatCh.YFP & $\begin{array}{l}\text { This paper: } \\
\text { Provided by Institut de la } \\
\text { vision, Paris France }\end{array}$ & plasmid \\
\hline AAV5-flox-EF1a-hCatCh-YFP & $\begin{array}{l}\text { This paper: } \\
\text { Provided by Institut de la } \\
\text { vision, Paris France }\end{array}$ & Virus (AAV) \\
\hline pAAV.Ef1a.DIO.Jaws.eGFP & Adgene & 65014 -Plasmid \\
\hline AAV5-CAG-Flex-Jaws-eGFP & $\begin{array}{l}\text { This paper: } \\
\text { Provided by Institut de la } \\
\text { vision, Paris France }\end{array}$ & Virus (AAV) \\
\hline pAAV-Ef1a-DIO-YFP & $\begin{array}{l}\text { This paper } \\
\text { Provided by Institut de la } \\
\text { vision, Paris France }\end{array}$ & Plasmid \\
\hline AAV5-CAG-Flex-Jaws-eGFP & $\begin{array}{l}\text { This paper } \\
\text { Provided by Institut de la } \\
\text { vision, Paris France }\end{array}$ & Virus (AAV) \\
\hline pAAV-Ef1a-DIO-YFP & Adgene & 105539-Plasmid \\
\hline AAV5-flox-EF1a-YFP & $\begin{array}{l}\text { This paper } \\
\text { Provided by Institut de la } \\
\text { vision, Paris France }\end{array}$ & Virus (AAV) \\
\hline pAAV-CAG-Jaws-KGC-GFP-ER2 & Adgene & 99233-Plasmid \\
\hline AAV2-CAG-Jaws-GFP & $\begin{array}{l}\text { This paper } \\
\text { Provided by Institut de la } \\
\text { vision, Paris France }\end{array}$ & Virus (AAV) \\
\hline
\end{tabular}




\begin{tabular}{|c|c|c|}
\hline pAAV-CAG-GFP & Adgene & 83279--Plasmid \\
\hline AAV2-7m8-CAG-GFP & $\begin{array}{l}\text { This paper } \\
\text { Provided by Institut de la } \\
\text { vision, Paris France }\end{array}$ & Virus (AAV) \\
\hline pAAV-flox-Ef1a-hChR2-YFP & $\begin{array}{l}\text { This paper } \\
\text { Provided by Institut de la } \\
\text { vision, Paris France }\end{array}$ & Plasmid \\
\hline AAV9-flox-Ef1a-hChR2-YFP & $\begin{array}{l}\text { This paper } \\
\text { Provided by Institut de la } \\
\text { vision, Paris France }\end{array}$ & Virus (AAV) \\
\hline AAV5-hSyn-hChR2(H134R)-eYFP & Adgene & 26973-AAV5 \\
\hline AAV5.hSyn.eGFP.WPRE.bGH & Adgene & 105539-AAV5 \\
\hline AAV5-hsyn-Jaws-KGC-GFP-ER2 & Adgene & 65014-AAV5 \\
\hline psAAV-hSyn-GRABDA2m & $\begin{array}{l}\text { Sun et al., } 2020 \\
\text { https://doi.org/10.1101/20 } \\
20.03 .28 .013722\end{array}$ & Plasmid \\
\hline AAV1-hSyn-GRABDA2m & $\begin{array}{l}\text { This paper } \\
\text { Provided by Institut de la } \\
\text { vision, Paris France }\end{array}$ & Virus (AAV) \\
\hline \multicolumn{3}{|l|}{ Chemicals, peptides, and recombinant proteins } \\
\hline $\mathrm{NaCl}$ & Sigma-Aldrich & S7653 \\
\hline $\mathrm{KCl}$ & Sigma-Aldrich & P9333 \\
\hline $\mathrm{NaH} 2 \mathrm{PO} 4$ & Sigma-Aldrich & S8282 \\
\hline $\mathrm{MgCl} 2$ & Sigma-Aldrich & M2670 \\
\hline $\mathrm{CaCl} 2$ & Sigma-Aldrich & 233506 \\
\hline $\mathrm{NaHCO} 3$ & Sigma-Aldrich & S6297 \\
\hline Sucrose & Sigma-Aldrich & S0389 \\
\hline Glucose & Sigma-Aldrich & 49159 \\
\hline Kynurenic Acid & Sigma-Aldrich & K3375 \\
\hline Albumin, from bovine serum & Sigma-Aldrich & A4503 \\
\hline KGlu & Sigma-Aldrich & P1847 \\
\hline HEPES & Sigma-Aldrich & H3375 \\
\hline EGTA & Sigma-Aldrich & E3889 \\
\hline ATP & Sigma-Aldrich & A9187 \\
\hline GTP & Sigma-Aldrich & G8877 \\
\hline Biocytin & Sigma-Aldrich & B4261 \\
\hline Nicotine tartrate & Sigma-Aldrich & N5260 \\
\hline Glucose & Sigma-Aldrich & G8270 \\
\hline DPBS 10x & Life Technologies & $14200-067$ \\
\hline Neurobiotin Tracer & Vector laboratories & SP-1120 \\
\hline Prolong Gold Antifade Reagent & Invitrogen & P36930 \\
\hline Chloral Hydrate & Sigma-Aldrich & $302-17-0$ \\
\hline Sodium Acetate & Sigma-Aldrich & 57654611 \\
\hline Quinpirole & Tocris & 55397 \\
\hline Eticlopride & Tocris & 57266 \\
\hline \multicolumn{3}{|l|}{ Critical commercial assays } \\
\hline EasyTag EXPRESS 35S Protein Labeling Kit & PerkinElmer & NEG772014MC \\
\hline
\end{tabular}




\begin{tabular}{|c|c|c|}
\hline CaspaseGlo 3/7 & Promega & G8090 \\
\hline TruSeq ChIP Sample Prep Kit & Illumina & IP-202-1012 \\
\hline \multicolumn{3}{|l|}{ Deposited data } \\
\hline Raw and analyzed data & This paper & GEO: GSE63473 \\
\hline B-RAF RBD (apo) structure & This paper & PDB: $5 J 17$ \\
\hline Human reference genome NCBI build 37, GRCh37 & $\begin{array}{l}\text { Genome Reference } \\
\text { Consortium }\end{array}$ & $\begin{array}{l}\text { http://www.ncbi.nlm. } \\
\text { nih.gov/projects/gen } \\
\text { ome/assembly/grc/h } \\
\text { uman/ }\end{array}$ \\
\hline Nanog STILT inference & $\begin{array}{l}\text { This paper; Mendeley } \\
\text { Data }\end{array}$ & $\begin{array}{l}\text { http://dx.doi.org/10. } \\
17632 / \text { wx6s4mj7s8. } \\
2\end{array}$ \\
\hline $\begin{array}{l}\text { Affinity-based mass spectrometry performed with } 57 \\
\text { genes }\end{array}$ & $\begin{array}{l}\text { This paper; Mendeley } \\
\text { Data }\end{array}$ & $\begin{array}{l}\text { Table S8; } \\
\text { http://dx.doi.org/10. } \\
\text { 17632/5hvpvspw82. } \\
1\end{array}$ \\
\hline \multicolumn{3}{|l|}{ Experimental models: Organisms/strains } \\
\hline $\begin{array}{l}\text { strain (mouse), strain background (mus musculus) - } \\
\text { males }\end{array}$ & $\begin{array}{l}\text { Janvier Laboratories, } \\
\text { France }\end{array}$ & $\begin{array}{l}\text { C57BI/6j SC-C57J- } \\
M\end{array}$ \\
\hline $\begin{array}{l}\text { strain (mouse), strain background (mus musculus) - } \\
\text { males }\end{array}$ & $\begin{array}{l}\text { Maskos et al., } 2005 \\
\text { https://doi.org/10.1038/374 } \\
065 \mathrm{a0}\end{array}$ & $\begin{array}{l}\text { ACNB2 KO } \\
\text { maintained on a } \\
\text { C57BL6/J backgrou } \\
\text { nd }\end{array}$ \\
\hline $\begin{array}{l}\text { strain (mouse), strain background (mus musculus) - } \\
\text { males }\end{array}$ & $\begin{array}{l}\text { Turiault et al., } 2007 \\
\text { https://doi.org/10.1111/j.17 } \\
42-4658.2007 .05886 . x\end{array}$ & $\begin{array}{l}\text { DATicre } \\
\text { maintained on a } \\
\text { C57BL6/J backgrou } \\
\text { nd }\end{array}$ \\
\hline \multicolumn{3}{|l|}{ Software and algorithms } \\
\hline R Project for Statistical Computing & http://www.r-project.org/ & RRID:SCR_001905 \\
\hline Fiji & http://fiji.sc & RRID:SCR_002285 \\
\hline PyCharm & $\begin{array}{l}\text { CE version } 2020.3 .4 \\
\text { (Python 3.8) }\end{array}$ & RRID:SCR_018221 \\
\hline Adobe Illustrator 2020 & Adobe & RRID:SCR_010279 \\
\hline Spike 2 Software & CED & RRID:SCR_000903 \\
\hline Spike sort 3D & 5.6 .3 & $\begin{array}{l}\text { Neuralynx } \\
\text { acquisition }\end{array}$ \\
\hline Spike extractor & 2.5.0.0 & $\begin{array}{l}\text { Neuralynx } \\
\text { acquisition }\end{array}$ \\
\hline Cheetah software & version 3.012 .5 .4 & $\begin{array}{l}\text { Neuralynx } \\
\text { acquisition }\end{array}$ \\
\hline Doric Neuroscience Studio & Doric & RRID:SCR_018569 \\
\hline Clampfit (pClamp suite) & Molecular Devices & RRID:SCR_011323 \\
\hline
\end{tabular}


CellPress 
A

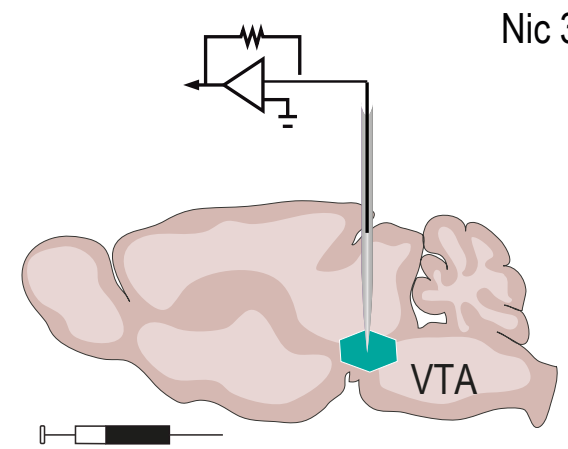

+ Nicotine (IV)

C

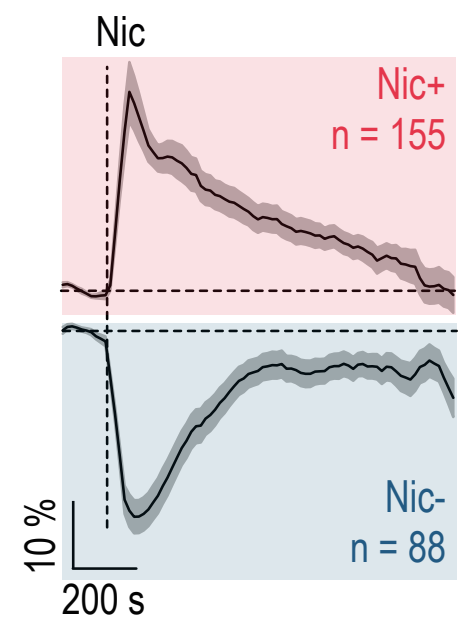

F

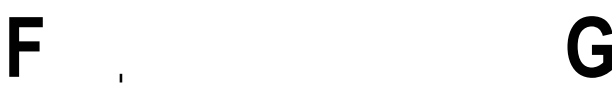

G
$30 \mu \mathrm{g} / \mathrm{kg}$

B Nicotine-evoked responses
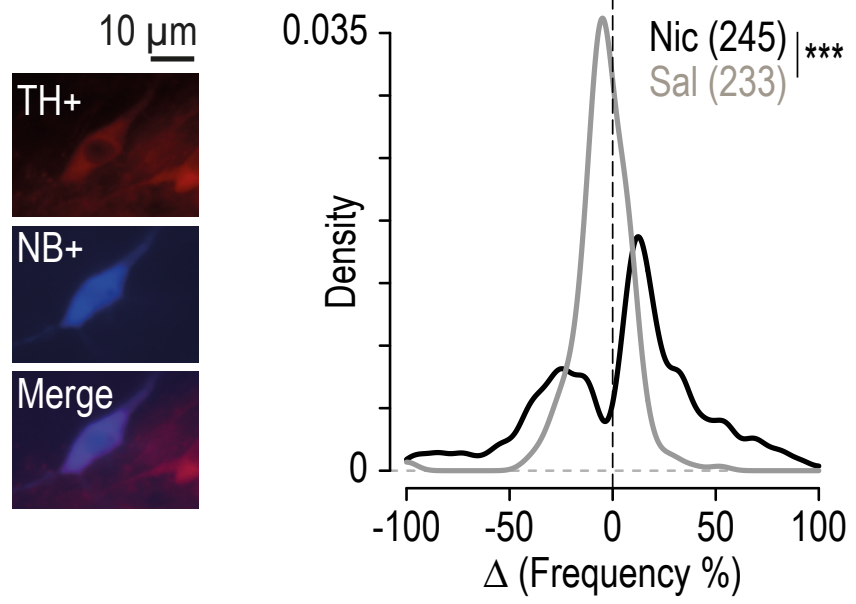

E
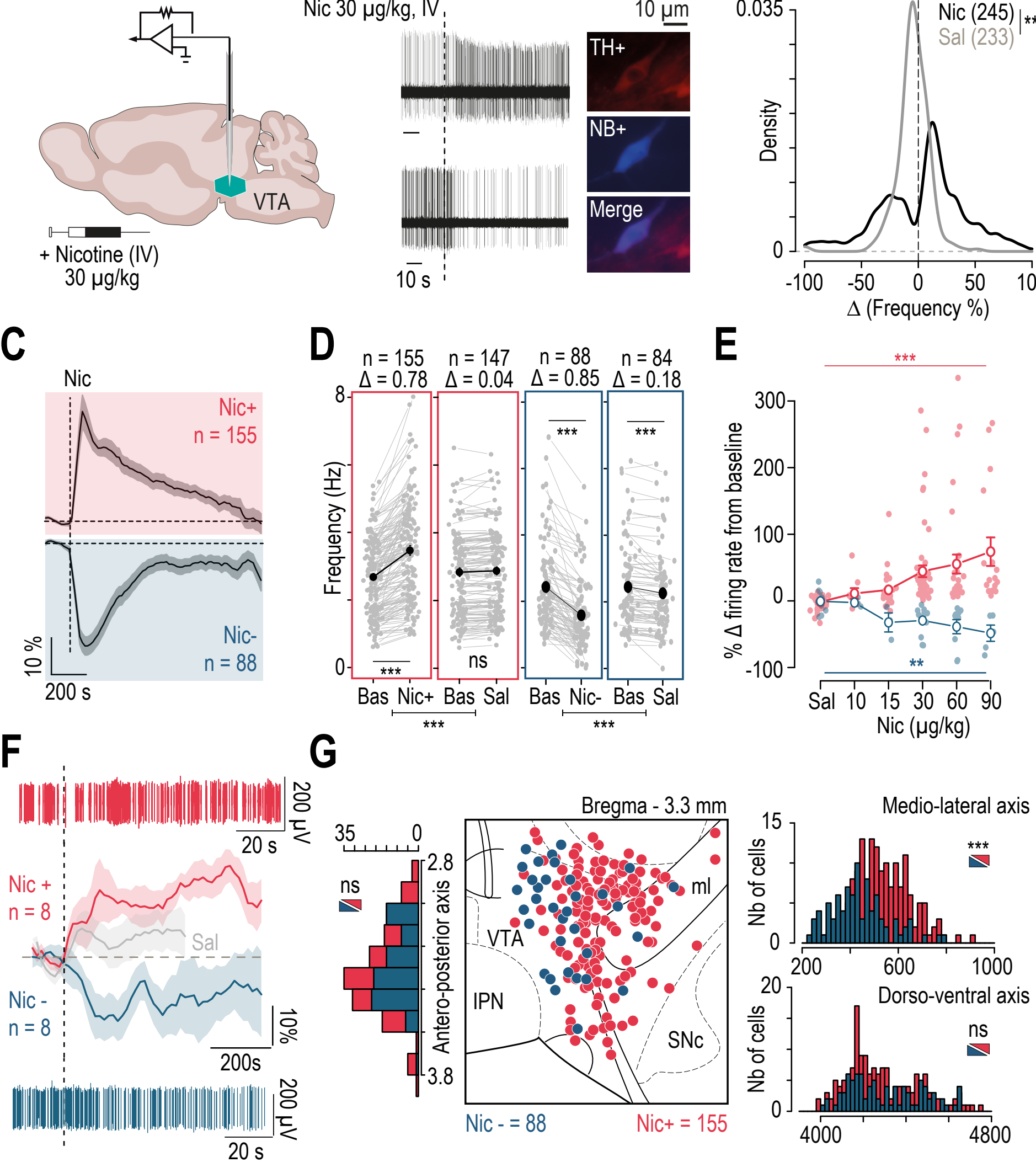

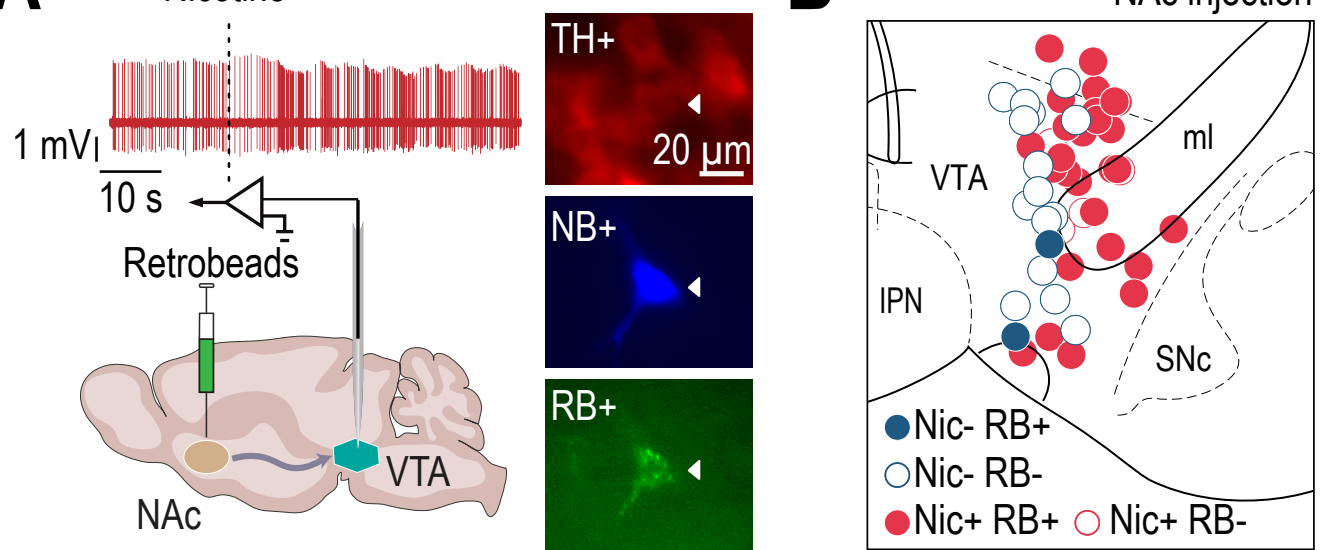

D + Nicotine

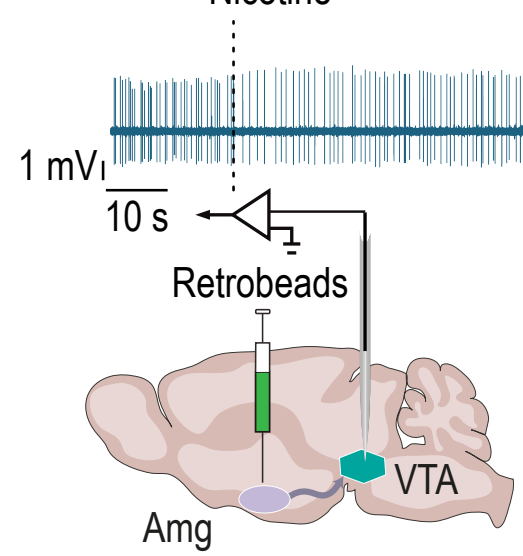

$\mathrm{TH}+$

E

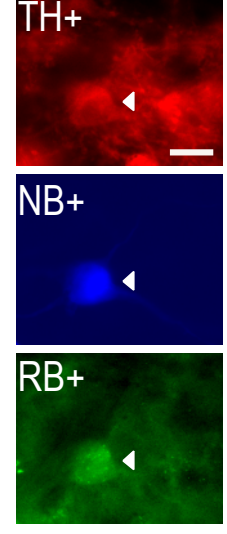

Amg injection
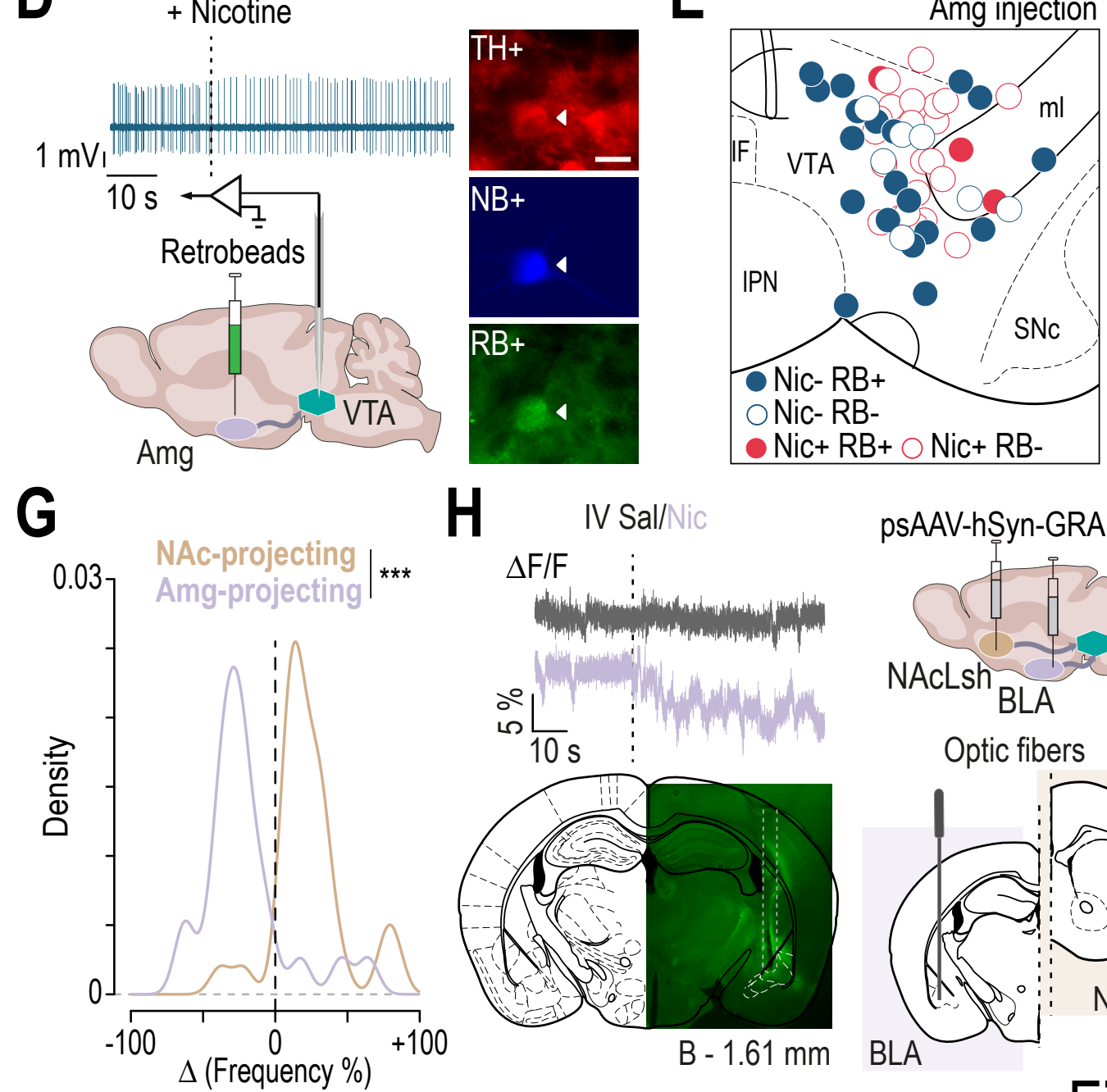

$\mathbf{H}_{\Delta \mathrm{F} / \mathrm{F}} \mathrm{IV} \mathrm{Sal/Nic}$

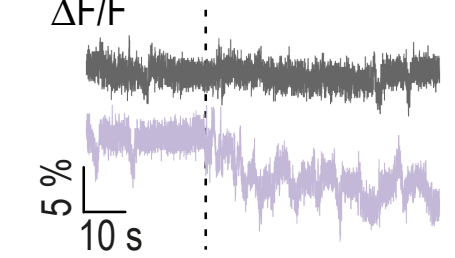

psAAV-hSyn-GRAB ${ }_{\text {DA2m }}$
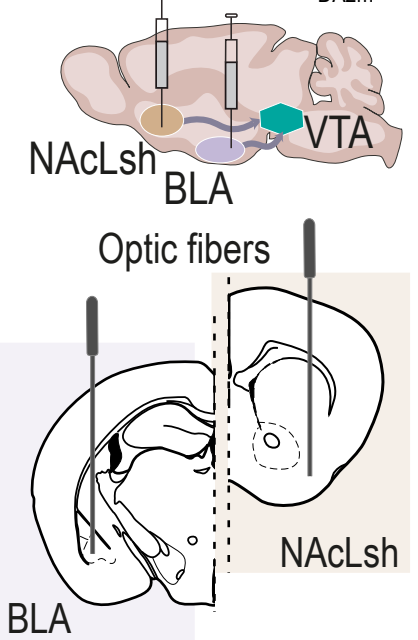

B - $1.61 \mathrm{~mm}$

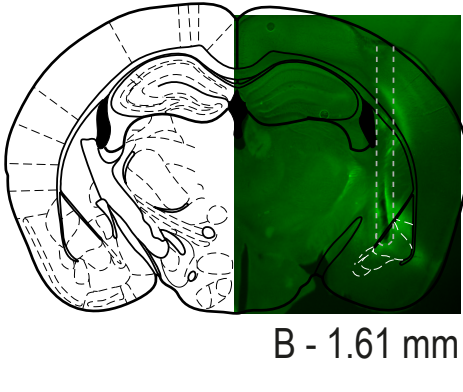

Figure 2

F
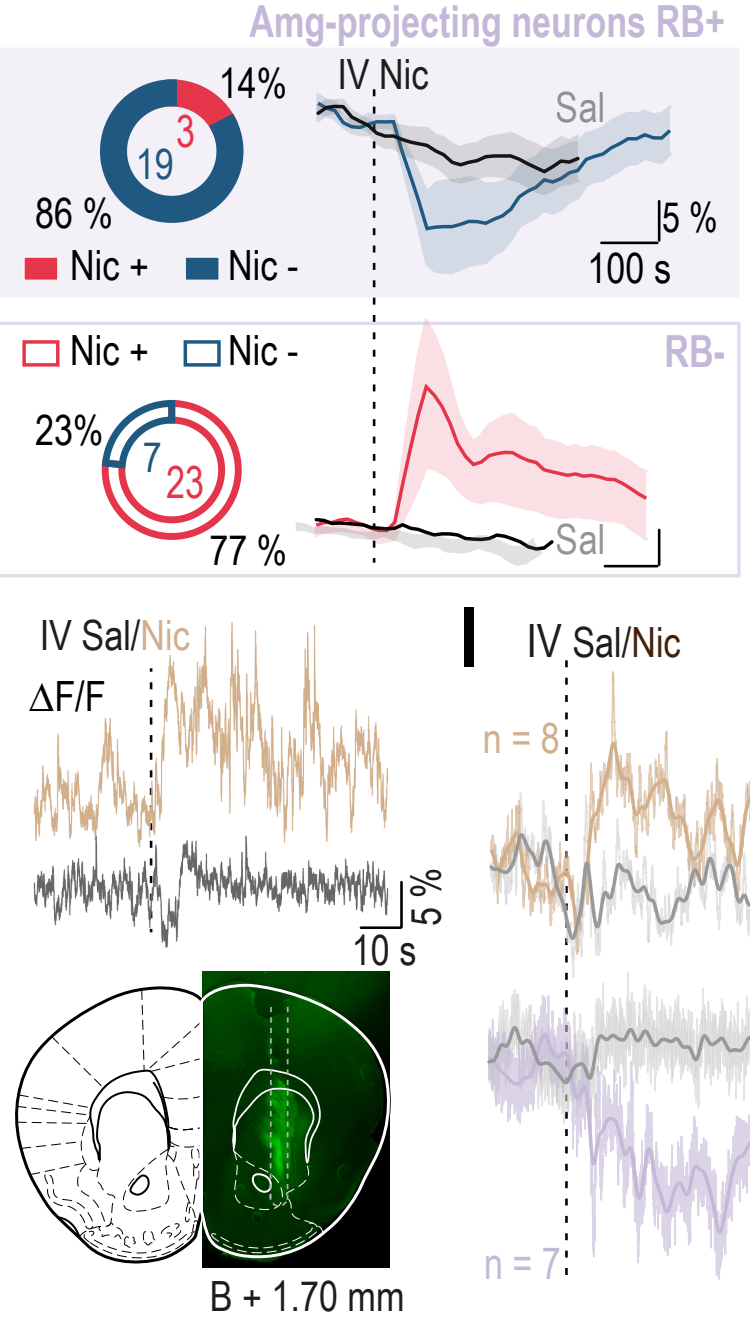

IV SalNic
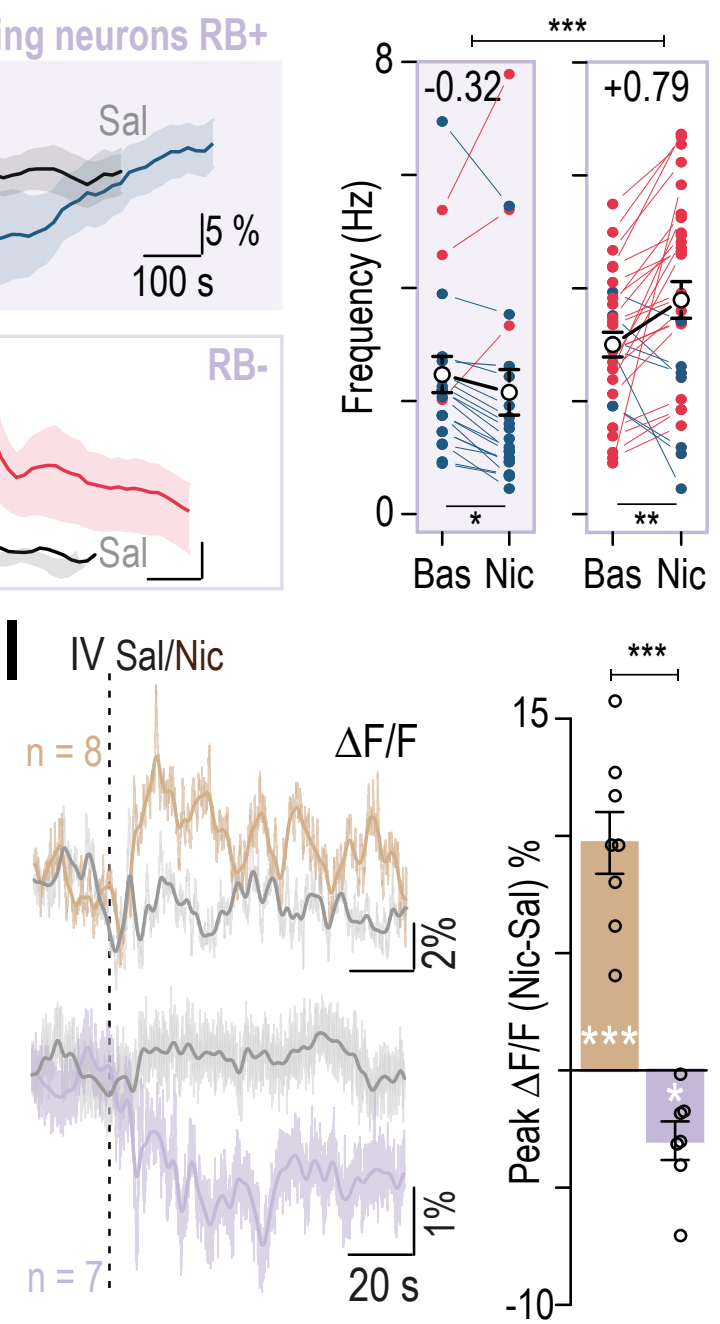
A
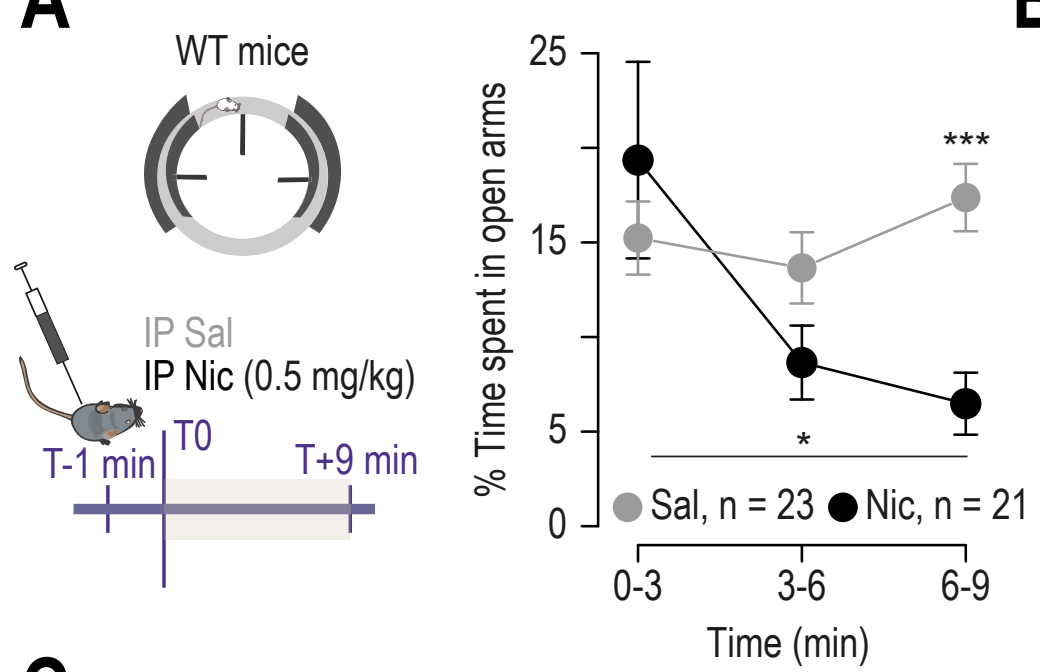

B

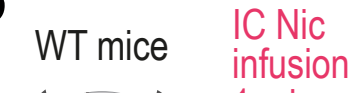

C IV Nic $30 \mu \mathrm{g} / \mathrm{kg}$

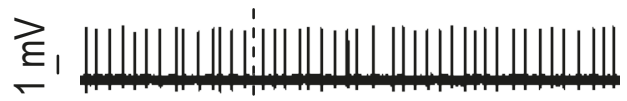

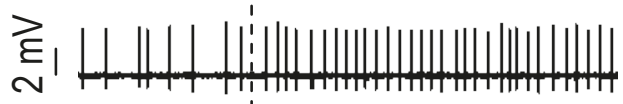

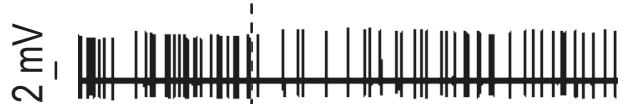
$\sim \mathrm{Nic} \frac{\mathrm{s}}{20}$
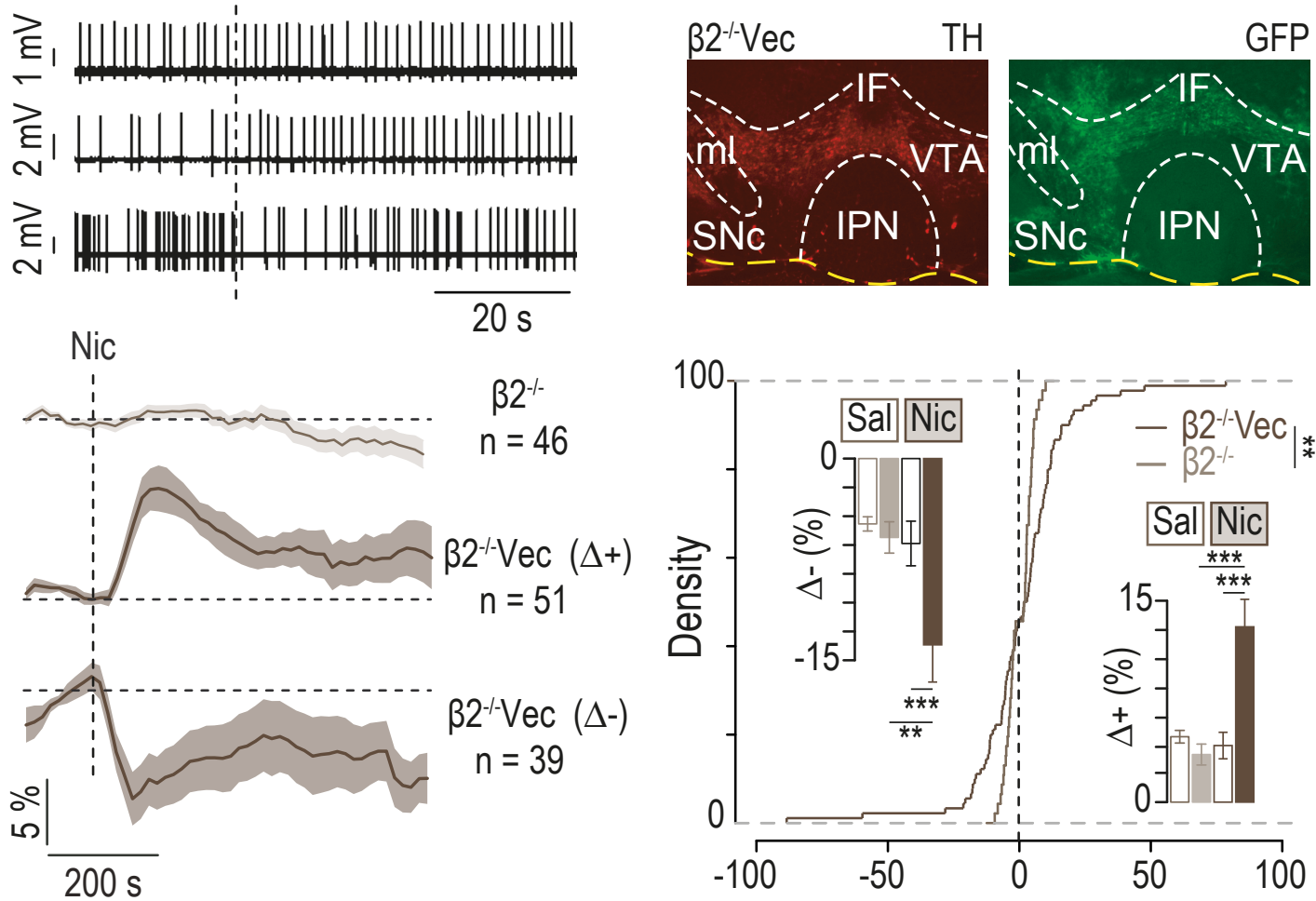

D
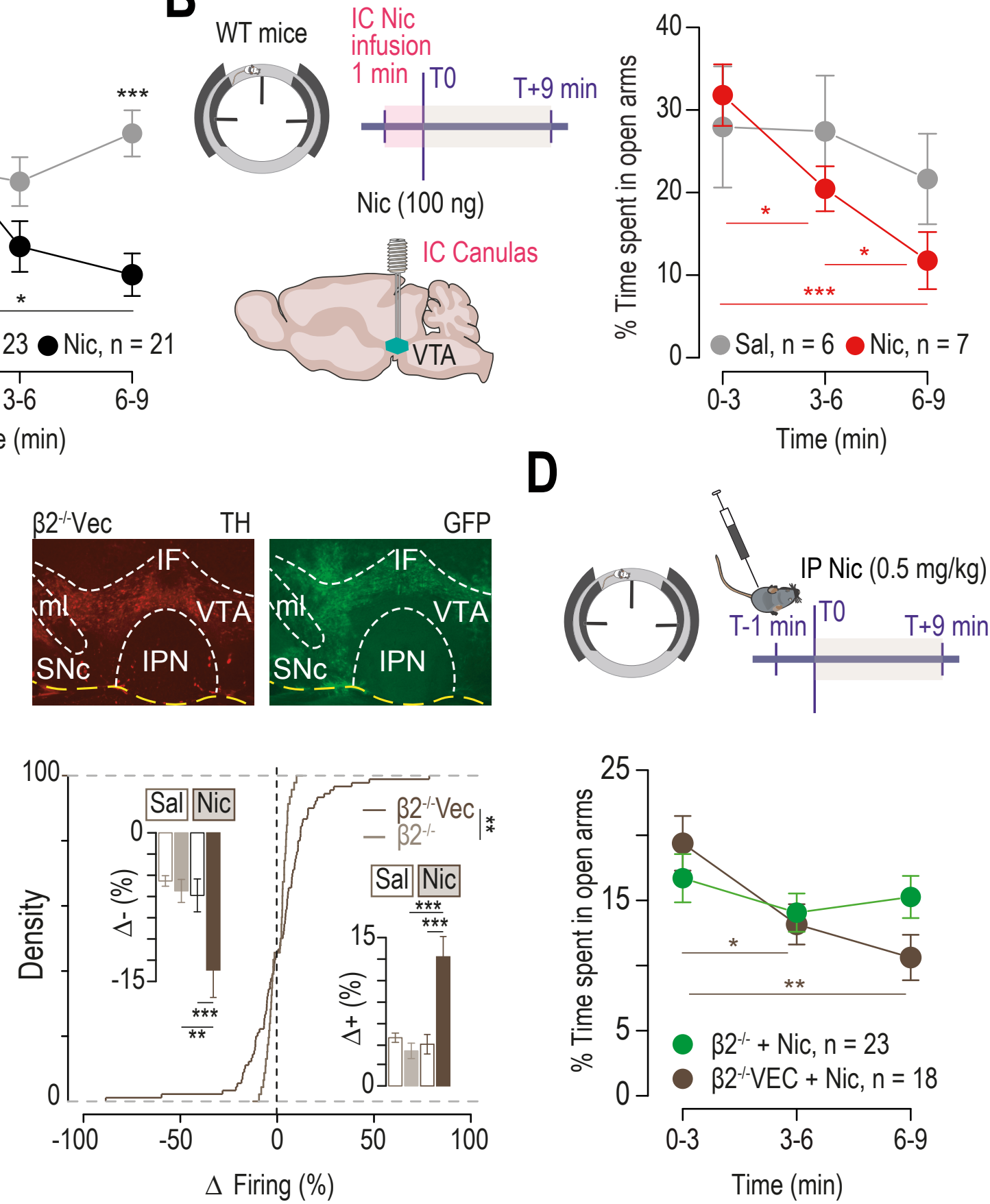

Figure 3 

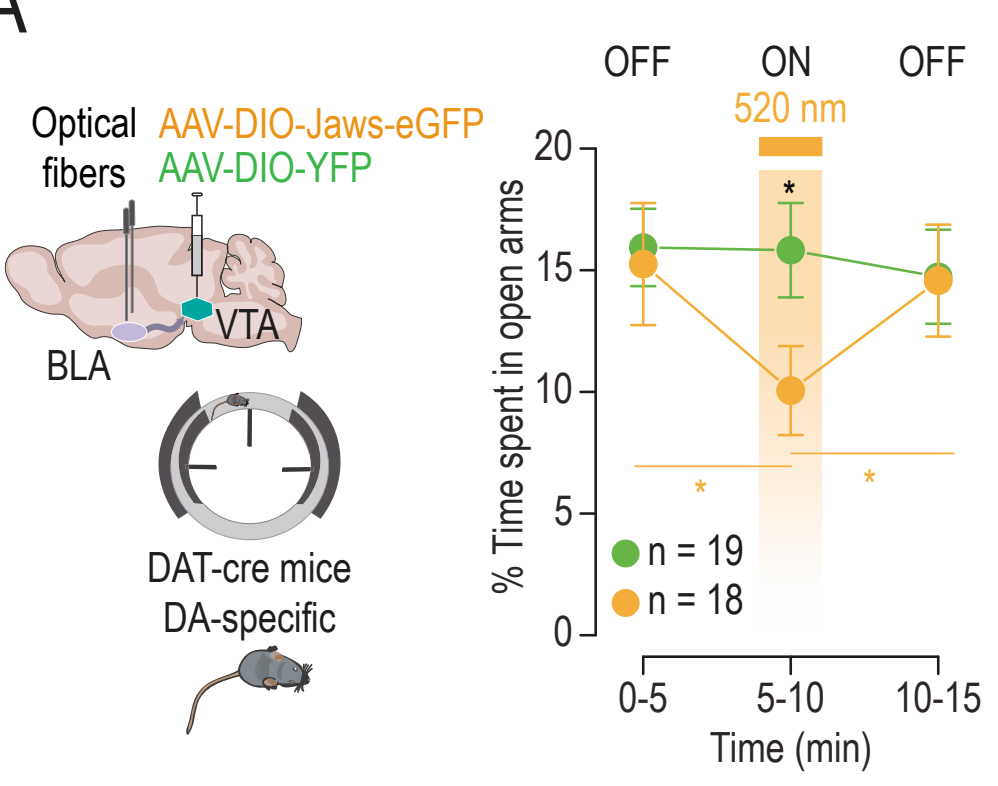

B

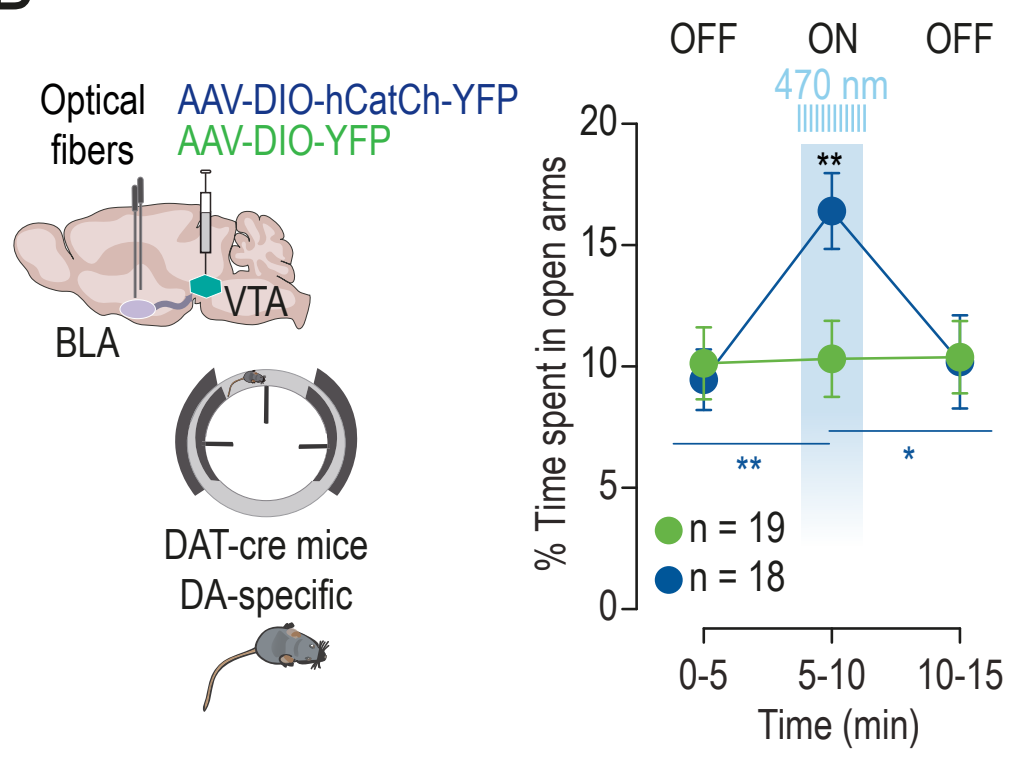

C

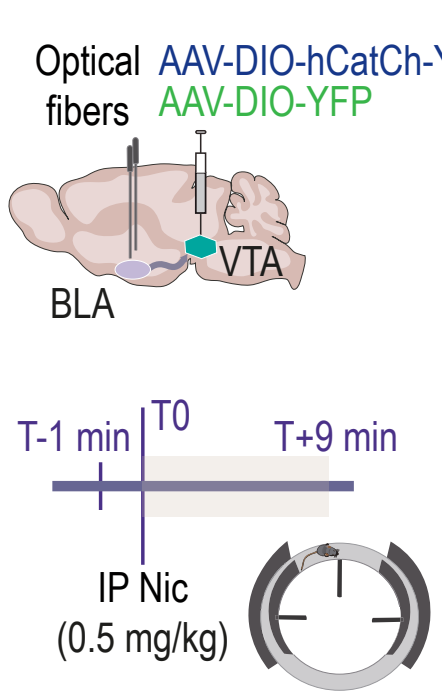

D

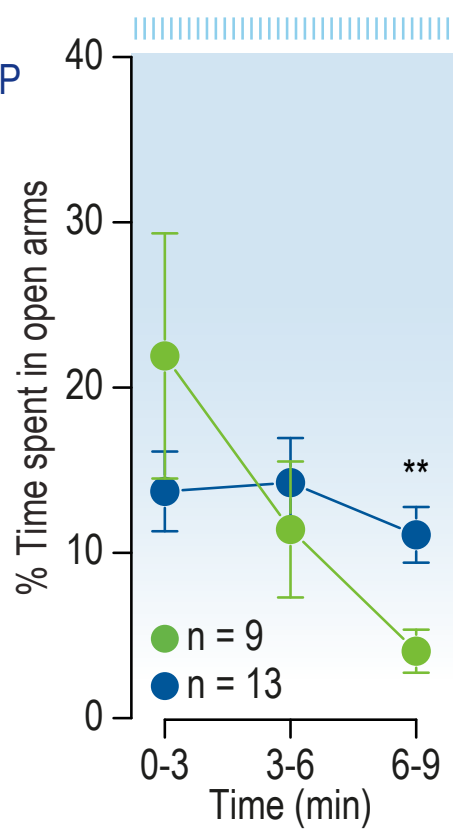

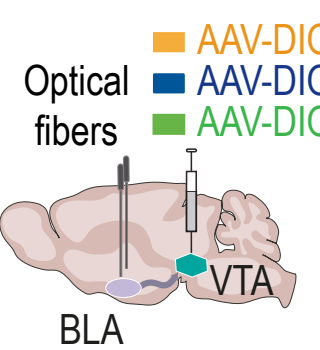

One-side continuous stim. $520 \mathrm{~nm}$

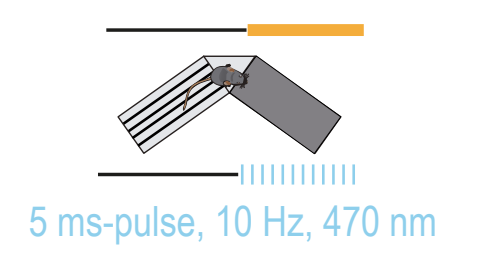

ns

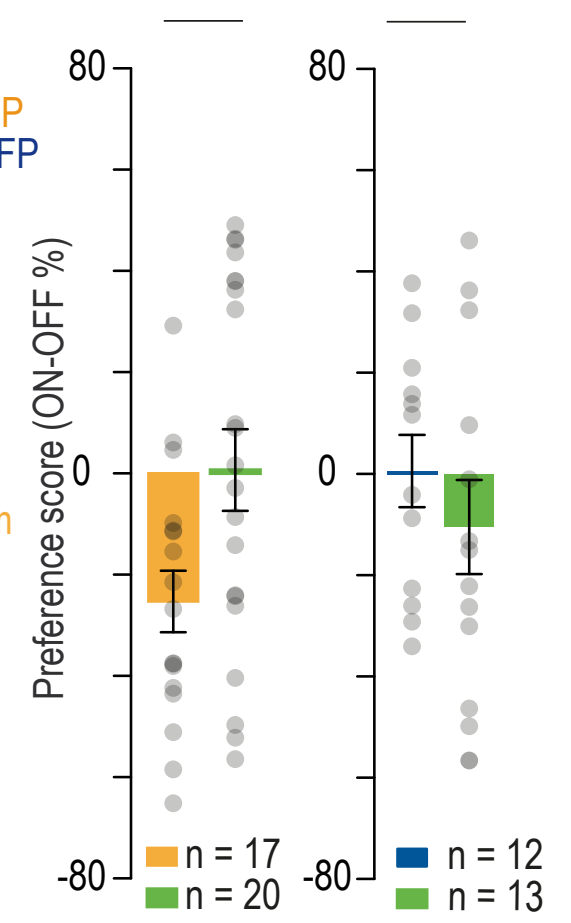

Figure 4 
A

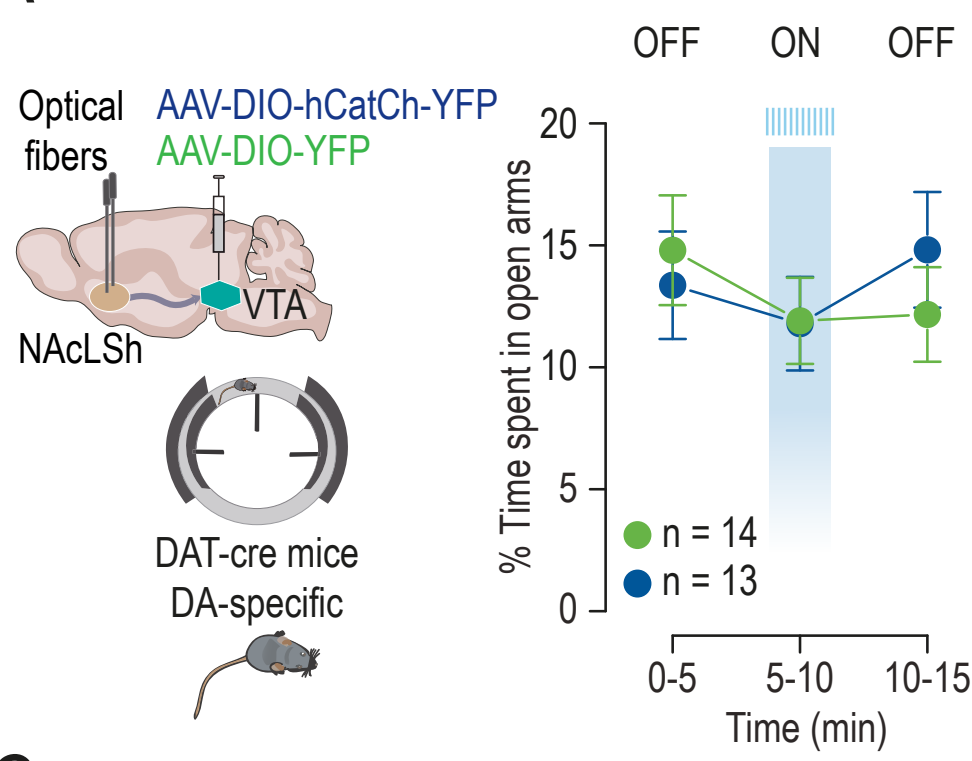

C

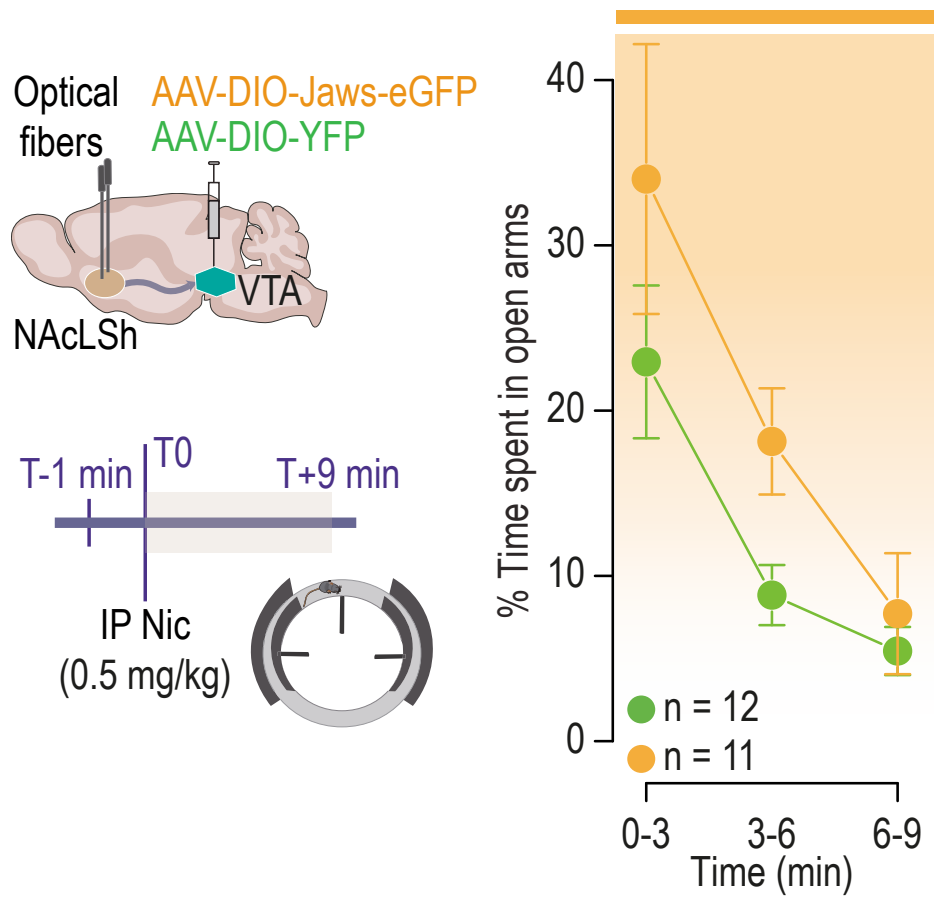

B

Optical AAV-DIO-Jaws-eGFP fibers AAV-DIO-YFP

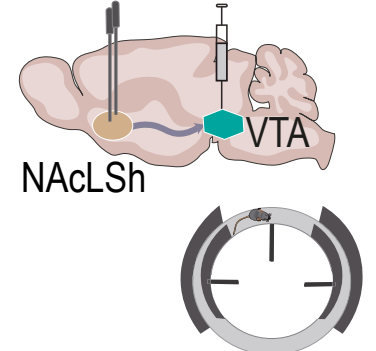

DAT-cre mice DA-specific

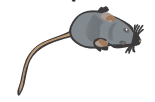

D
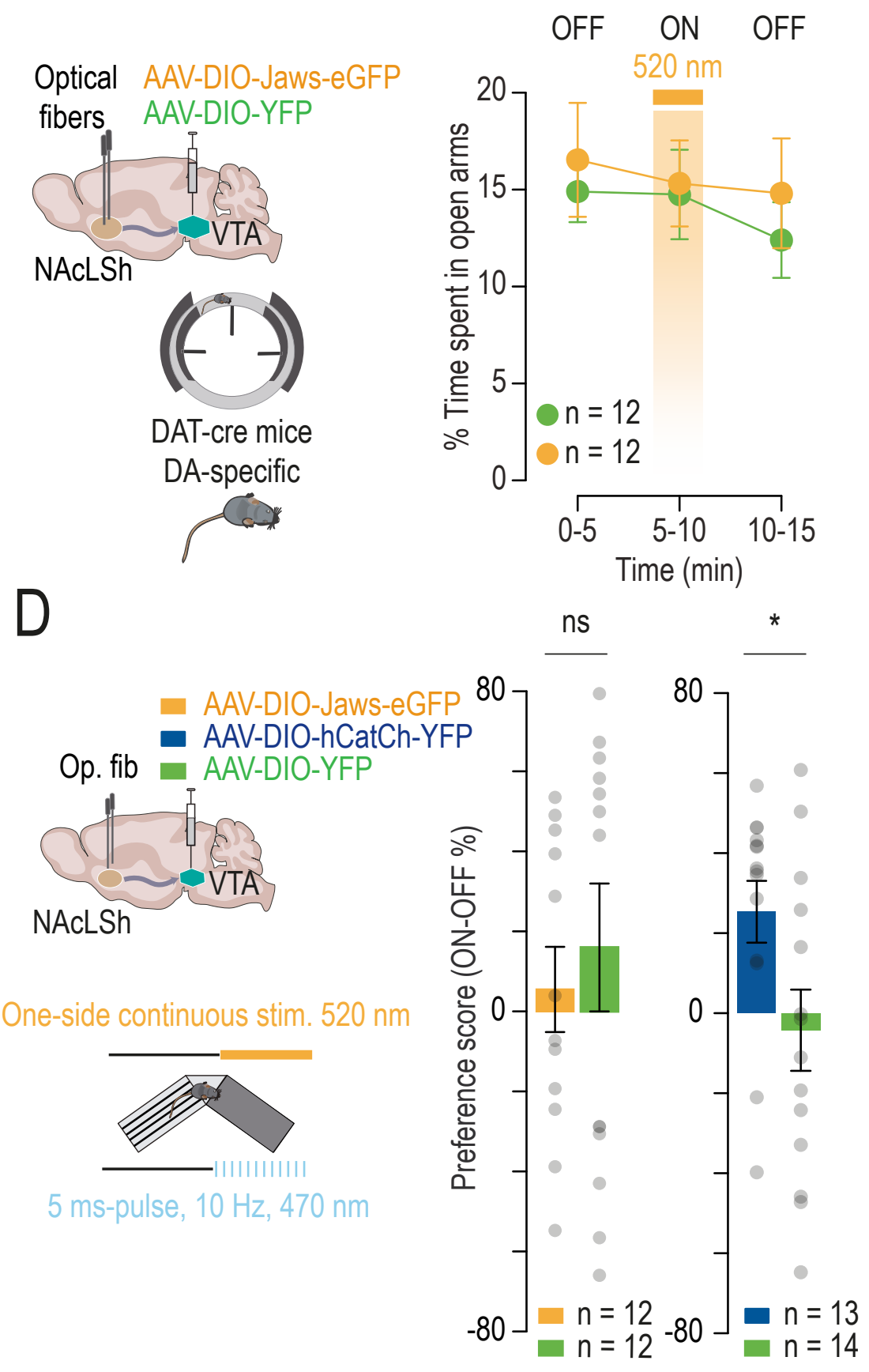

Figure 5 


\section{Supplemental Information}

\section{Nicotine inhibits the VTA to Amygdala dopamine pathway to promote anxiety \\ Nguyen C, Mondoloni S, Le Borgne T, Centeno I, Come M, Jehl J, Solié C, Reynolds LM, Durand-de Cuttoli R, Tolu S, Valverde S, Didienne S, Hannesse B, Fiancette JF, Pons S, Maskos U, Deroche-Gamonet V, Dalkara D, Hardelin JP, Mourot A, Marti F \& Faure P}

Supplemental Information consists of:

Supplementary Figure S1, related to Figure 1.

Supplementary Figure S2, related to Figure 1.

Supplementary Figure S3, related to Figure 1.

Supplementary Figure S4, related to Figure 2.

Supplementary Figure S5, related to Figure 2.

Supplementary Figure S6, related to Figure 2.

Supplementary Figure S7, related to Figure 3.

Supplementary Figure S8, related to Figure 3.

Supplementary Figure $\$ 9$, related to Figure 4.

Supplementary Figure S10, related to Figure 4.

Supplementary Figure S11, related to Figure 4.

Supplementary Figure S12, related to Figure 4.

Supplementary Figure S13, related to Figure 5.

Supplementary Figure S14, related to Figure 5. 
Figure S1: Paired responses to saline and nicotine injections of in vivo recorded DA neurons in anesthetized mice. Related to Figure 1.

Responses of VTA DA neurons to saline (left) and nicotine (right) injections. Responses are rank ordered based on the response to nicotine, from the most inhibited (below) to the most excited (above). Left: Colored scale of amplitude responses. Right: Examples of individual responses to nicotine (10 neurons), horizontal dotted lines indicate 0 , vertical dotted lines the nicotine injections. 

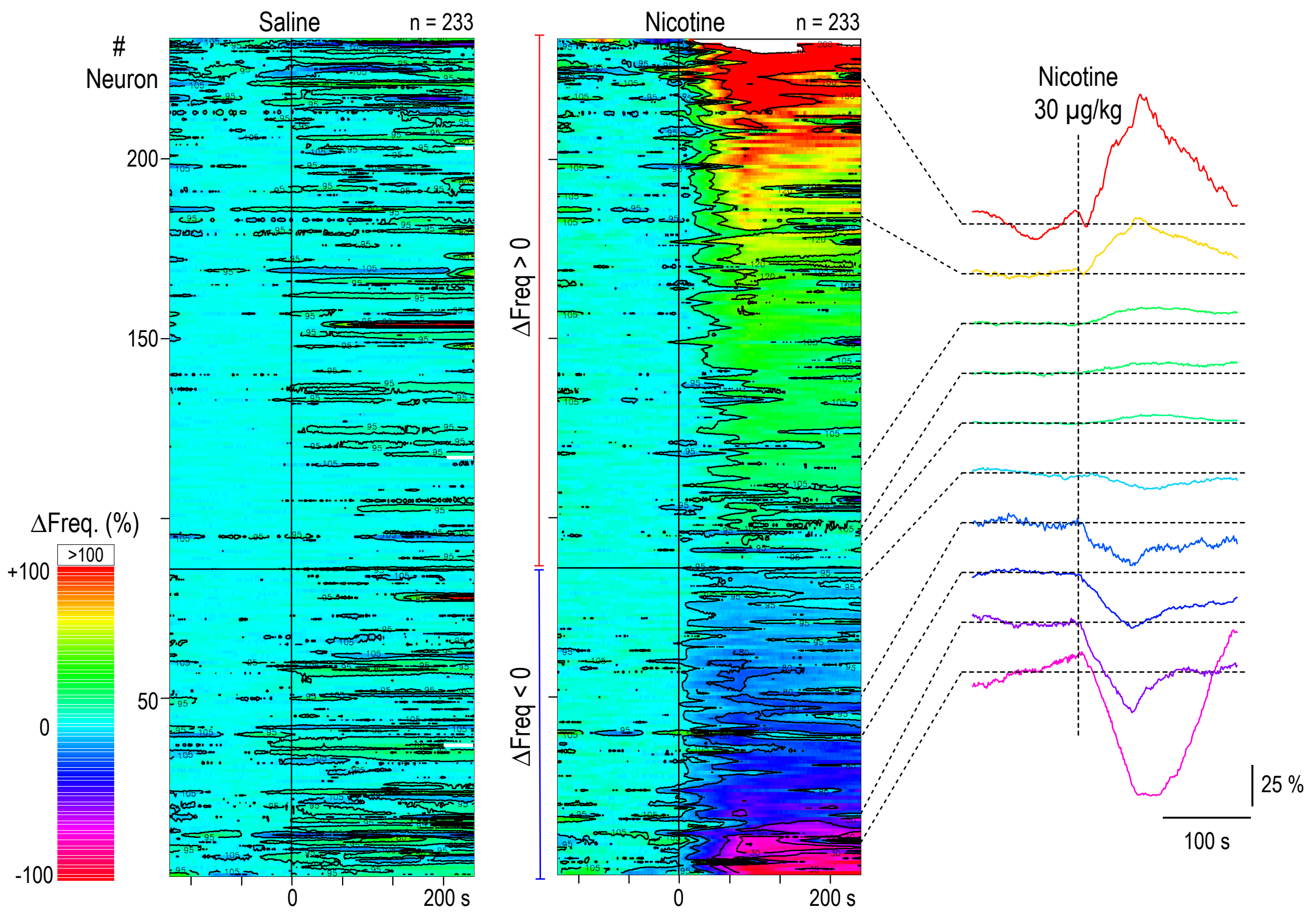

Figure S1 
Figure S2: In vivo recordings of DA neurons: anesthetized and freely moving experiments. Related to Figure 1.

(A) From left to right: Picture of the micro-drive (upside-down view). Schematic of micro-drive implantation for in vivo recordings of freely moving mice. Histological slice showing tetrode implantation in the VTA. (B) Left: Schematic of the plastic cylinder used for IV nicotine injection into the tail vein during tetrode and fiber photometry recordings in freely moving animals. Right: Example of one multi-channel recording of three VTA neurons (color-coded after clustering on all channels, top). GABAergic neurons are in green and blue and a putative DA neuron is in red. DA neurons are characterized by electrophysiological criteria (firing frequency, bursting activity, regularity of firing and large refractory period as seen in the auto-correlogram, bottom) and their response to Quinpirole/Eticlopride (D2R selective agonist and antagonist respectively, bottom right). (C) Analysis of the spontaneous activity of NB-labeled DA neurons that were either activated (red) or inhibited (blue) by the nicotine injection. Top: Basal firing rates and percentage of spikes-within-burst (\%SWB) between activated and inhibited neurons were not statistically different (Wilcoxon test $p=0.051$ and $p=0.064$ ). Bottom: Interval between SWB (in ms) as a function of the length of the burst (from two to seven action potentials). 
A
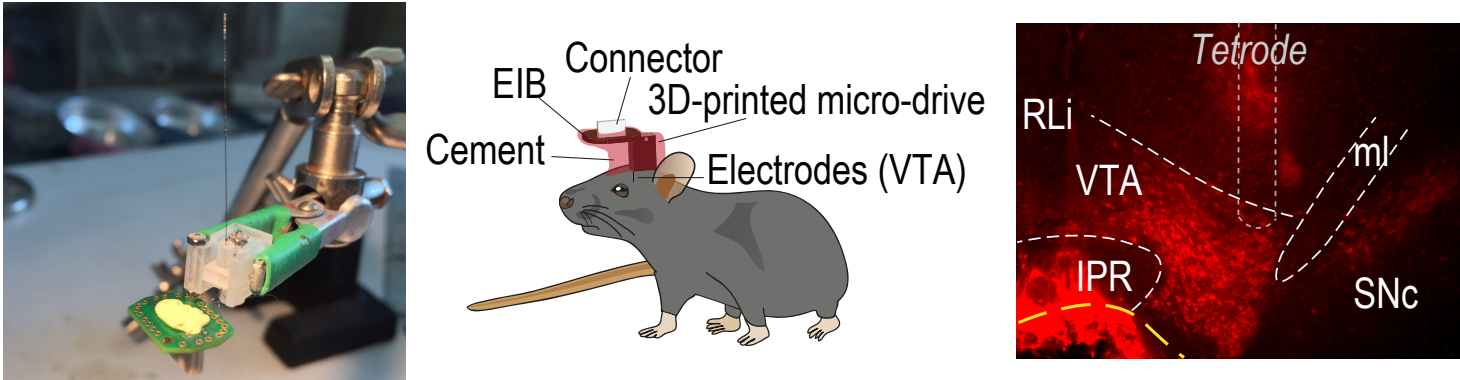

B
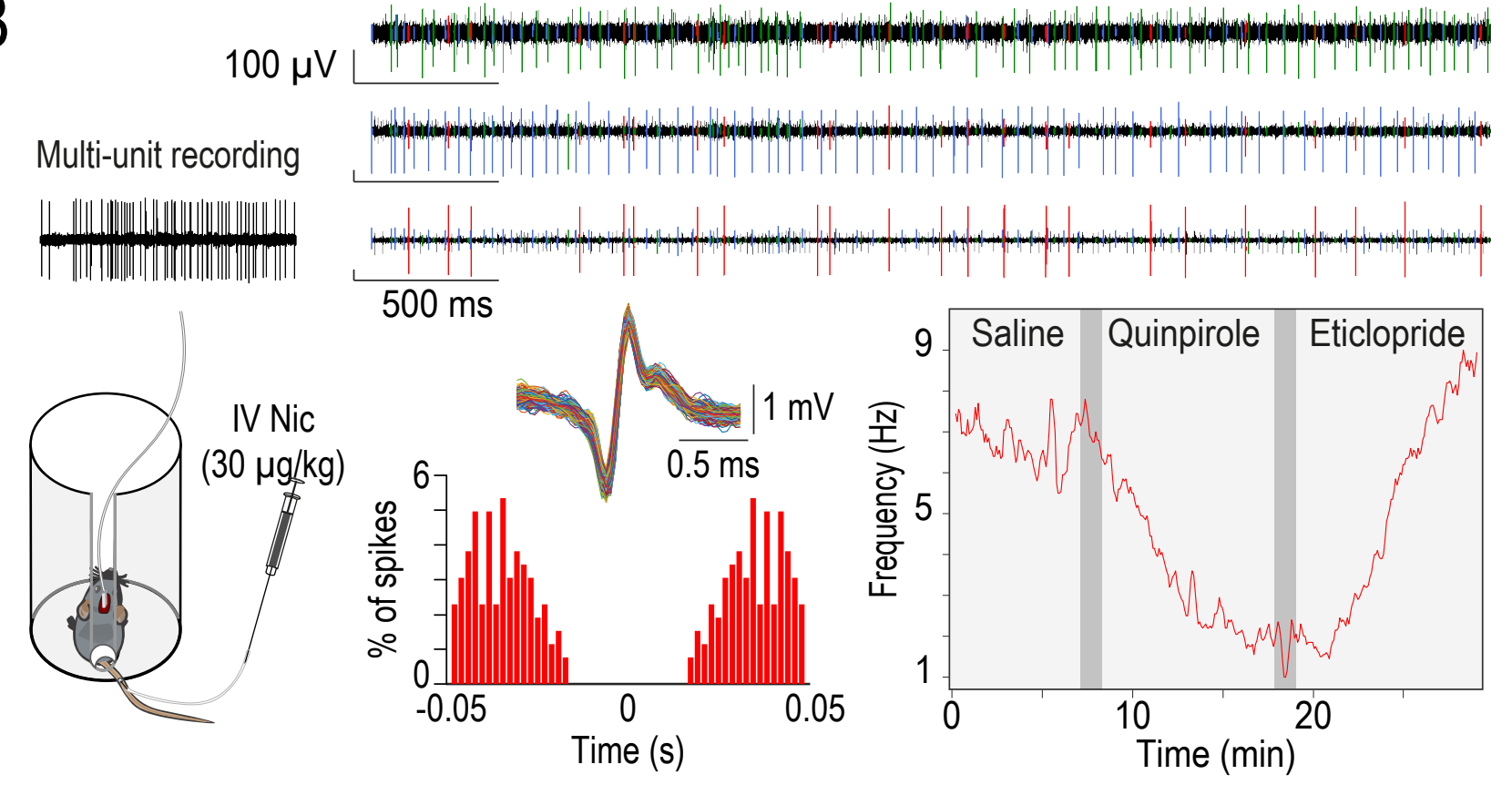

C
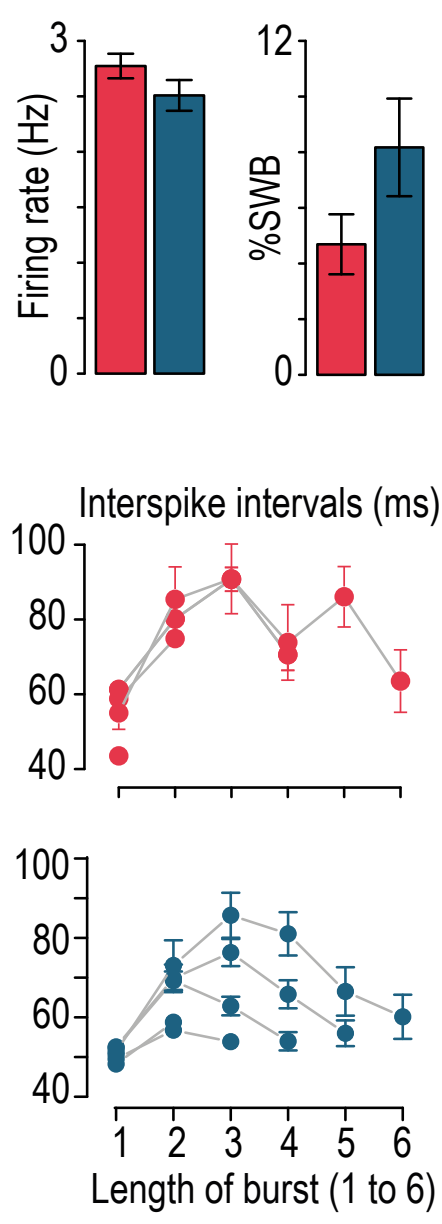

Figure S2 
Figure S3: Localization of in vivo labeled DA neurons on anesthetized mice. Related to Figure 1. Localization of VTA DA neurons labeled in vivo after juxtacellular recordings on anesthetized animals. Neurons are color-coded according to their responses to nicotine injection (activated in red, $n=155$ and inhibited in blue, $n=88$ ), and positioned according to the antero-posterior axis on the Paxinos atlas from Bregma -2.8 to $-3.8 \mathrm{~mm}$. 


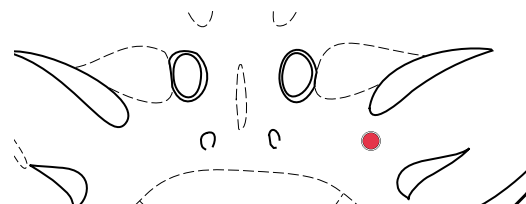

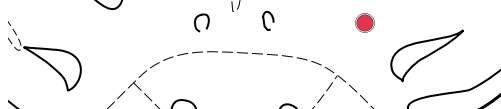
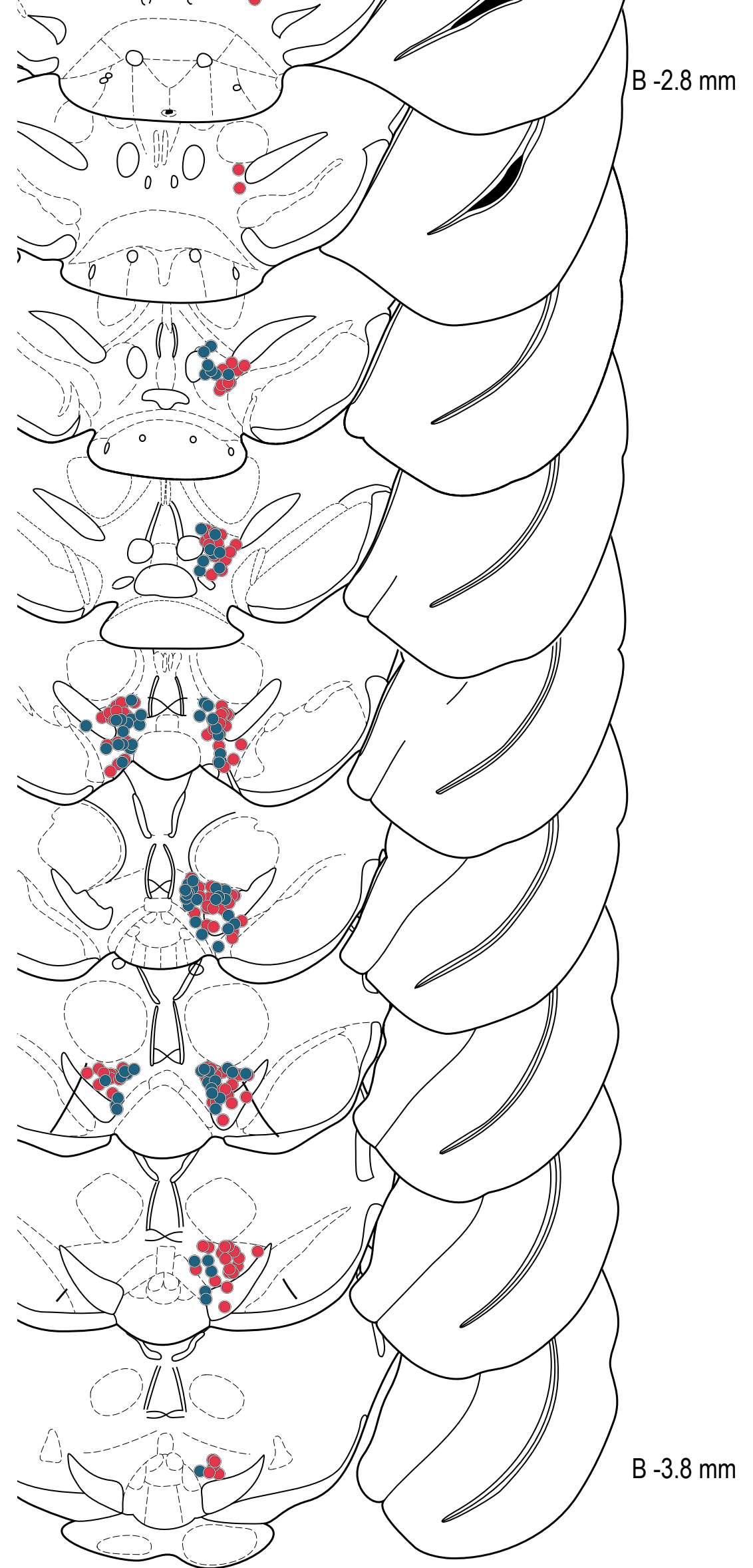

$$
(\mathrm{Nic}-)=88 \quad(\mathrm{Nic}+)=155
$$

Figure S3 
Figure S4: Retrobead injections into the NAc and Amg. Related to Figure 2.

(A, C) Examples of retrograde tracer (retrobeads RB, in yellow) injected in all of the NAc (A) and Amg sites (C), reported onto different Paxinos atlas slices. (B, D) Representative immunofluorescence images of VTA slices $(T H+$, red) revealing neurons containing $R B(R B+$, green), after $R B$ injection into the $N A c(B)$ or into the Amg (D). (E) Left: A Paxinos atlas slice at $3.3 \mathrm{~mm}$ from bregma, onto which neurobiotin-filled cell bodies of all recorded neurons from RB-injected mice were positioned (NAc-projecting neurons $n=30$, gold and Amg-projecting neurons $n=22$, purple). Right: Medio-lateral distribution (shown as density) of the neurons shown in E) that project either to the NAc (gold) or to the Amg (purple). Amg-projecting neurons were located more medially in the VTA than NAc-projecting neurons (Wilcoxon test, $\left.{ }^{* * *} p<0.001\right)$. (F) Analysis of the spontaneous activity of NB-labeled DA neurons that were found RB+ after either NAc injection ( $n=30$, gold) or Amg injection ( $n=22$, purple). NAc-projecting DA neurons and Amg-projecting DA neurons displayed similar firing frequency (Wilcoxon test, $p=0.8$ ). The Amg-projecting neurons tend to have a higher bursting activity even though it is not statistically different than the NAc-projecting neurons (Wilcoxon test, $p=0.28$ ). IF: interfascicular nucleus; IPN: interpeduncular nucleus; ml: medial lemniscus; SNc: substantia nigra pars compacta 
A

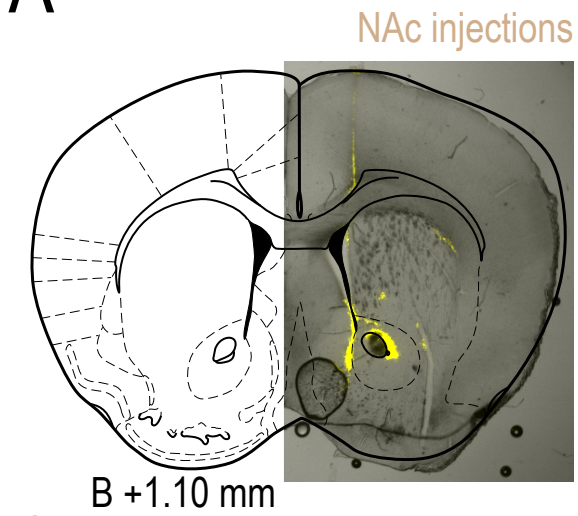

C

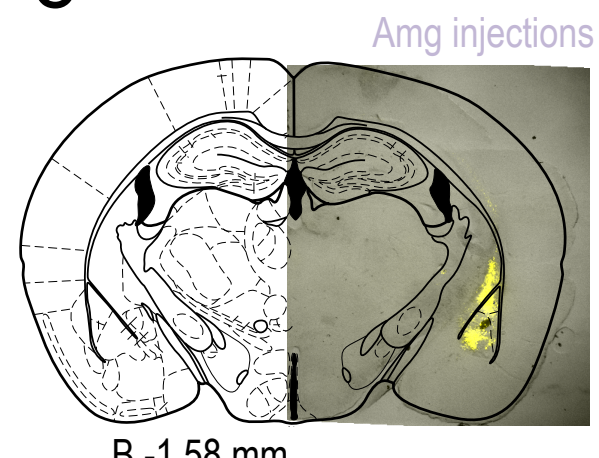

B $-1.58 \mathrm{~mm}$

E

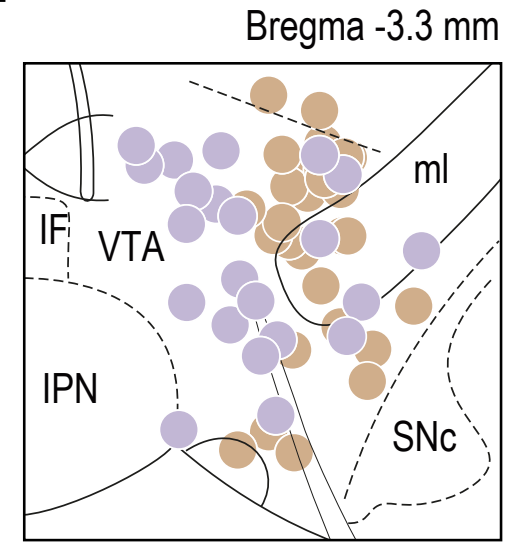

B

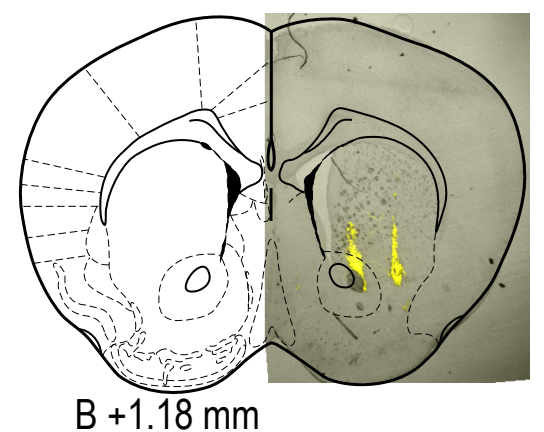

IF

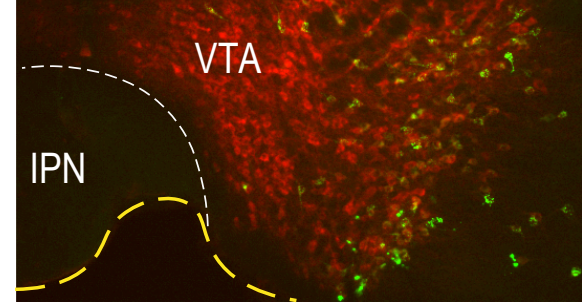

D
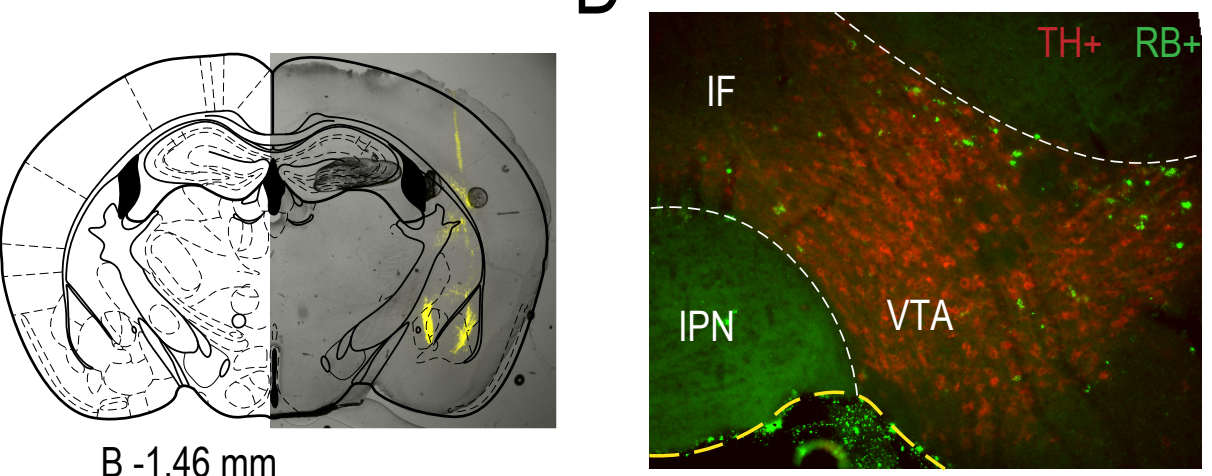

F

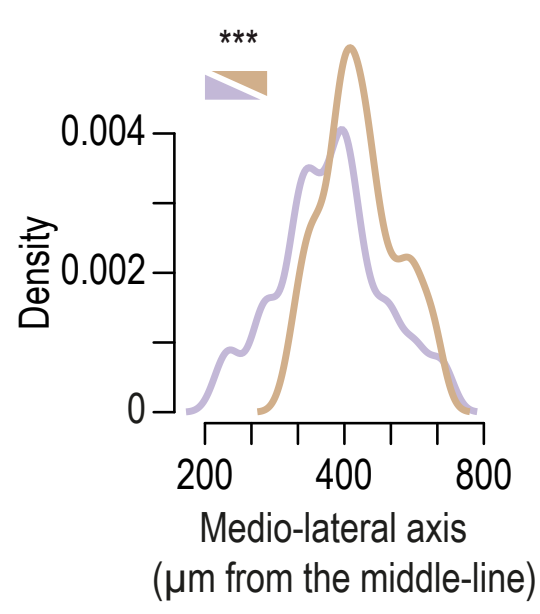

Figure S4 
Figure S5: DA neurons projecting to either NAcMSh or NAcLSh are activated by nicotine injection. Related to Figure 2.

(A) Retrobeads (RB) were injected in the NAc Medial Shell (NAcMSh) of WT mice and VTA DA neuron responses to an IV nicotine injection (30 $\mu \mathrm{g} / \mathrm{kg}$ ) were recorded in vivo on anesthetized animals, and labeled with neurobiotin. Example of RB site in the NAcMSh (top) and retrobeads labelling in the VTA two weeks after injection (bottom). (B) Post hoc identification of NAcMSh-projecting DA neurons by immunofluorescent colabeling of tyrosine hydroxylase (TH), neurobiotin (NB) and RB (scale bar $20 \mu \mathrm{m}$ ). Localization on the Paxinos atlas at bregma - $3.4 \mathrm{~mm}$ of NB-labeled DA neurons $(\mathrm{NB}+\mathrm{TH}+, n=22)$ following RB injection ( $\bullet \mathrm{RB}+, \circ \mathrm{RB}-$ ) into the NAcMSh. Red and blue colors denote nicotine-activated (Nic+) and nicotine-inhibited (Nic-) neurons, respectively. (RB+Nic+, $n=14, \mathrm{RB}+\mathrm{Nic}-, \mathrm{n}=0$; RB- Nic,$+ n=5 ; \mathrm{RB}-\mathrm{Nic}-, n=3)$. (C) Top: Percentage and number of Nic+ (red) and Nic- (blue) cells among NAcMSh-projecting DA neurons (RB+). Mean change in firing frequency of NAcMSh-projecting DA neurons in response to an IV injection of nicotine (red) or saline (grey). Bottom: Percentage and number of Nic+ (red) and Nic- (blue) cells in non-RB-labeled neurons (RB-). Mean change in firing frequency of RB- DA neurons in response to an IV injection of nicotine (black) or saline (grey). (D) Firing rate variation ( $\Delta$ ) from baseline (Bas) induced by nicotine (Nic) injection in RB+ (left, mean $\Delta=+0.50 \mathrm{~Hz}$ ) or RB- (right, mean $\Delta=+0.05 \mathrm{~Hz}$ ) DA neurons. (Comparison between mean firing rate during baseline and maximum firing rate after injection: paired Wilcoxon test, ${ }^{* * *} p(R B+)<0.001, p(R B-)=0.64$; Comparison between nicotine-induced firing rate variation evoked in RB+ and RB- DA neurons: Wilcoxon test, ${ }^{* *} p=0.004$ ). Mean scores are represented in black, and individual scores in red or blue. (E) RB were injected in the NAc Lateral Shell (NAcLSh) of WT mice and VTA DA neuron responses to an IV nicotine injection were recorded as in $(A)$. (F) Localization of NB-labeled DA neurons (NB+ $T H+, n=12$ ) following $R B$ injection into the NAcLSh. (RB+Nic+, $n=6 ; R B+N i c-, n=0 ;$ RB- Nic+, $n=4 ;$ RB- Nic-, $n=3$ ) and example of NB- labelled identified DA neuron (scale bar $20 \mu \mathrm{m}$ ). (G) Same as in (C) for NAcLSh -projecting (RB+) DA neurons (top) and for non-RB-labeled (RB-) neurons (bottom) when RB were injected in the NAcLSh. (H) Same as in (D) for NAcLSh -projecting (RB+) DA neurons (left, mean $\Delta=+0.73 \mathrm{~Hz}$ ) and for non-RB-labeled (RB-) neurons (right, mean $\Delta=-0.23 \mathrm{~Hz}$ ). (Comparison between mean firing rate during baseline and maximum firing rate after injection: paired Wilcoxon test, ${ }^{*} p(R B+)=0.03, p(R B-)=0.8$; Comparison between nicotine-induced firing rate variation evoked in RB+ and RB- DA neurons: Wilcoxon test, $p=0.13$ ). Mean scores are represented in black, and individual scores in red or blue. 
A
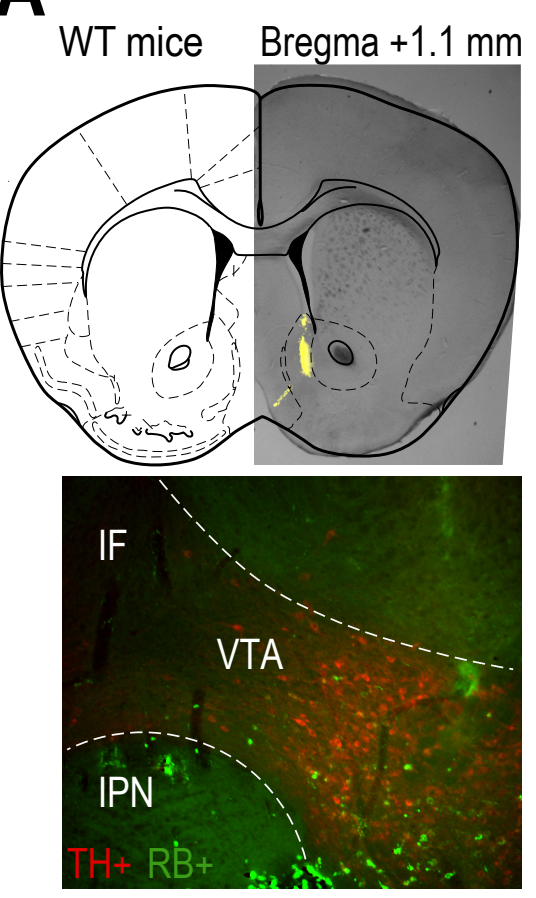

$E$

WT mice Bregma $+1.1 \mathrm{~mm}$
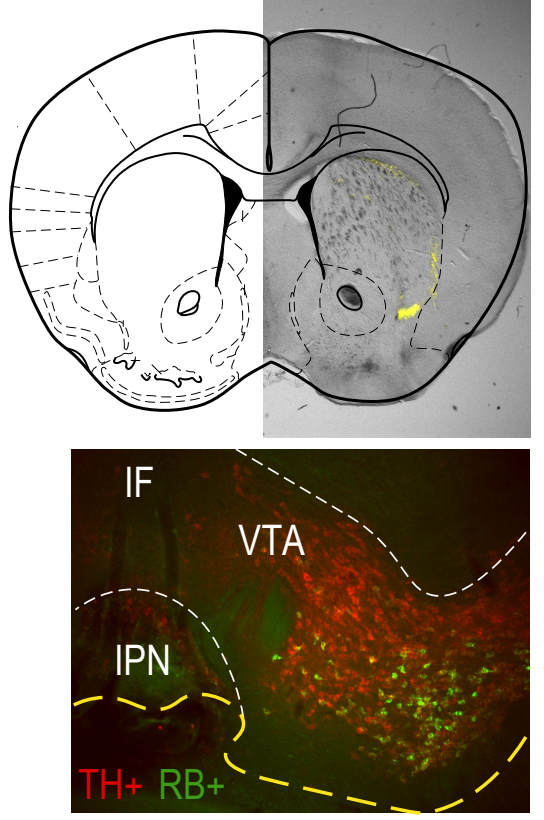

B
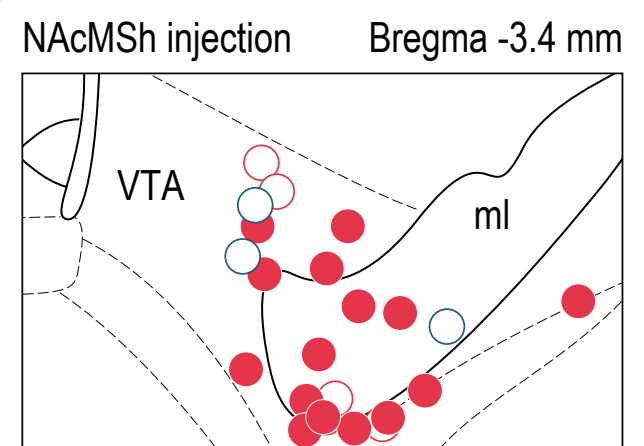

IPN

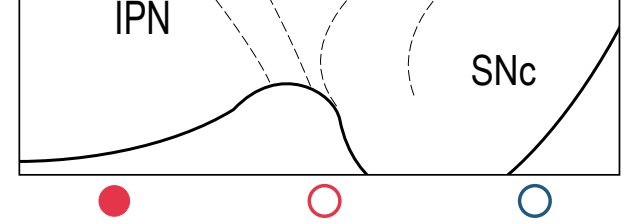

$\mathrm{Nic}+\mathrm{RB}+\mathrm{TH}+\mathrm{Nic}+\mathrm{RB}-\mathrm{TH}+\mathrm{Nic}-\mathrm{RB}-\mathrm{TH}+$

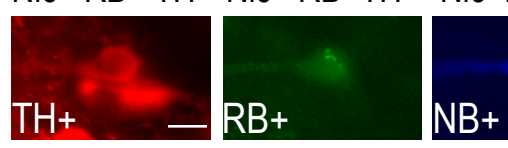

$\mathbf{F}$
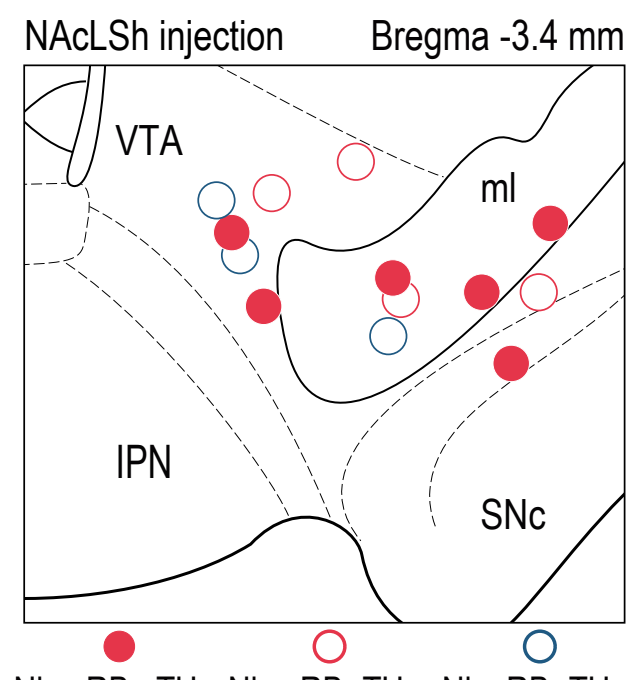

$\mathrm{Nic}+\mathrm{RB}+\mathrm{TH}+\mathrm{Nic}+\mathrm{RB}-\mathrm{TH}+\mathrm{Nic}-\mathrm{RB}-\mathrm{TH}+$

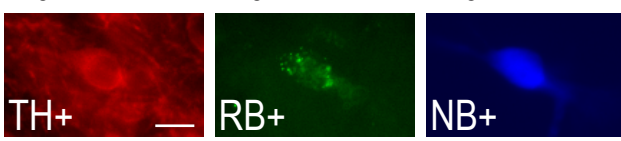

NAcMSh-projecting neurons RB+
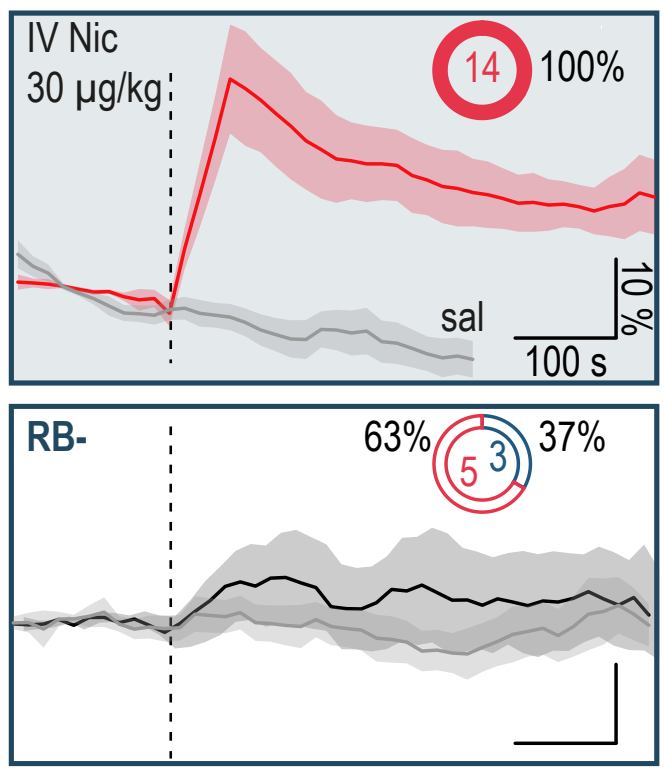

G
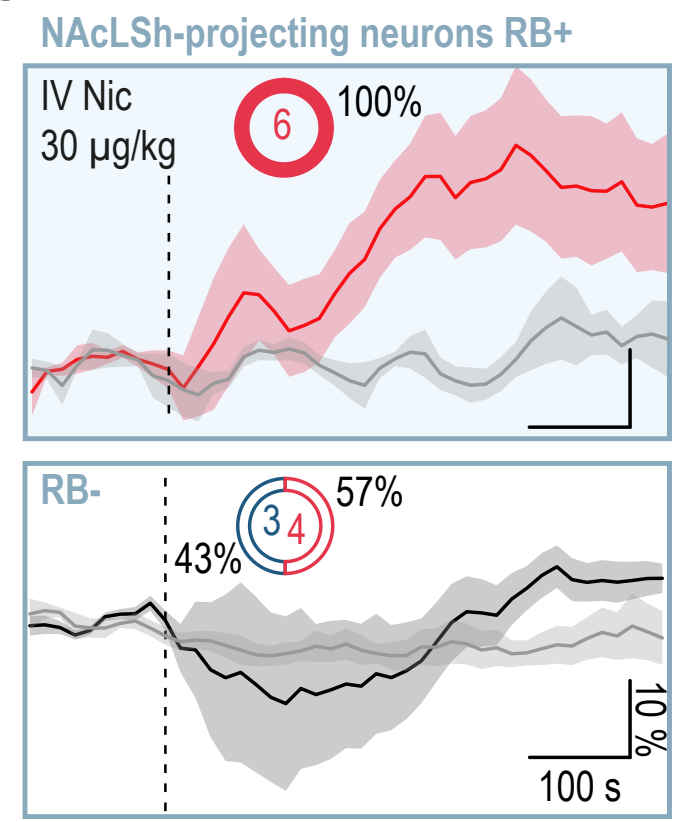

D

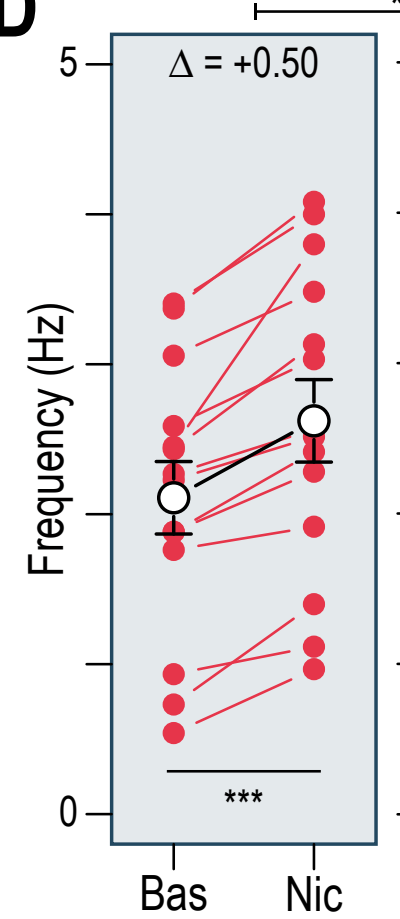

H

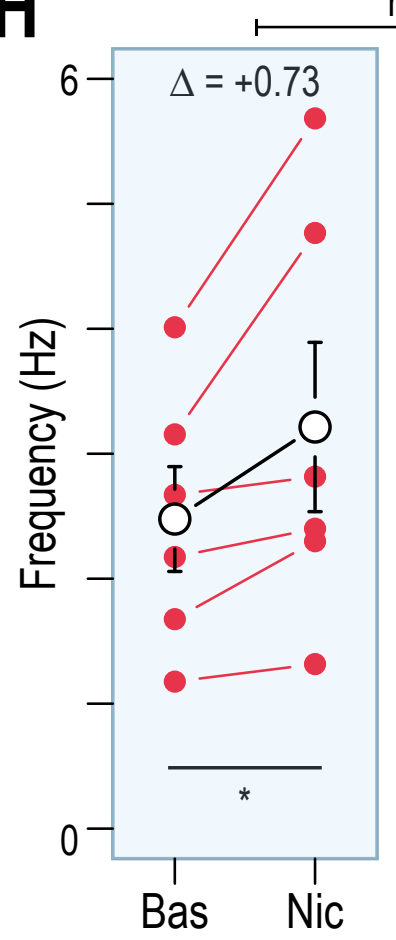

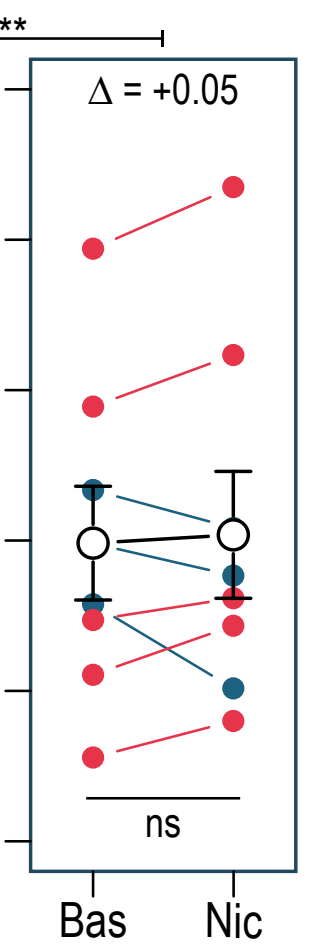

ns

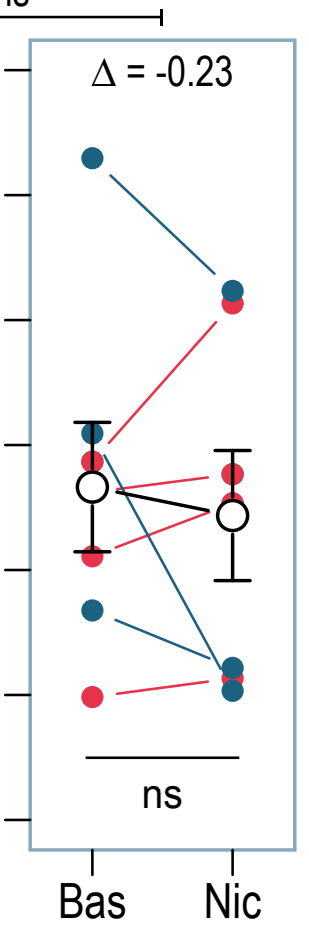

Figure S5 
Figure S6: VTA DA neurons projecting to the Amg are more excitable but not more reactive to nicotine than NAC-projecting DA neurons. Related to Figure 2.

(A) Immunofluorescence image of VTA DA ( $\mathrm{TH}+$ ) neurons projecting to the NAc (retrobeads, $\mathrm{RB}+$ ) labeled after patch-clamp recording (neurobiotin, $\mathrm{NB}+$ ). (B) Immunofluorescence image of an Amg-projecting (RB+) VTA DA $(\mathrm{TH}+$ ) neuron labeled after patch-clamp recording (neurobiotin, NB+). (C) Firing of NAc-projecting and Amg-projecting VTA DA neurons after current injections (20,60 and 100 pA). (D) Higher excitability of Amg-projecting ( $n=15$, purple) compared to NAc-projecting ( $n=13$, gold) DA neurons (two- way RM ANOVA main effect phenotype $F(1,26)=4.96,{ }^{*} p=0.035$, current $F(4,104)=15.97,{ }^{* * *} p<0.001$, current $x$ phenotype interaction $\left.F(4,104)=13.78,{ }^{* * *} p<0.001\right)$. (E) Nicotine-evoked currents (local puff $100 \mu \mathrm{M}$ ) in RB+-identified, NAc- or Amg-projecting VTA DA neurons recorded in brain slices (whole-cell voltage-clamp mode -60 mV). (F) Mean currents evoked by nicotine in either NAc-projecting ( $n=16$, gold, $33.0 \pm 19.8 \mathrm{pA})$ or Amg-projecting $(n=17$, purple, $22.4 \pm 13.3 p A)$ VTA DA neurons were not statistically different (Student's t-test, $p=0.08$ ). 
A

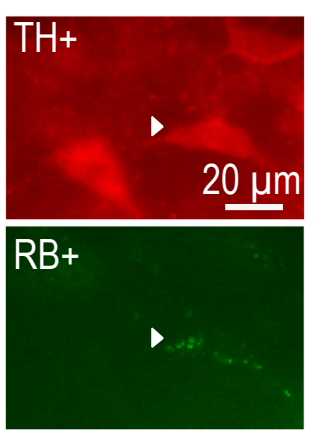

C

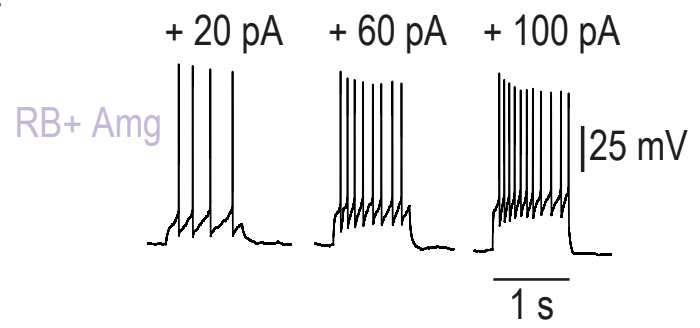

.

E

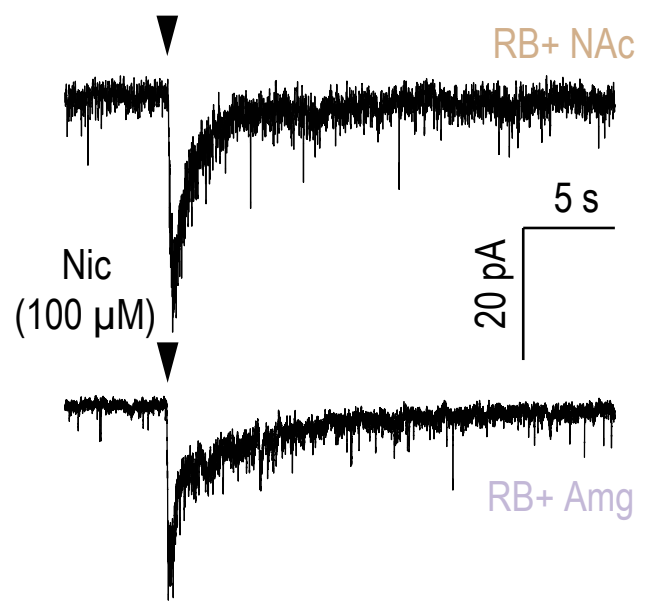

B

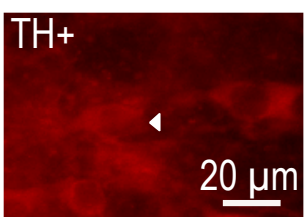

NB+
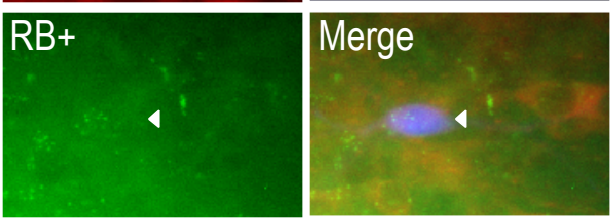

D

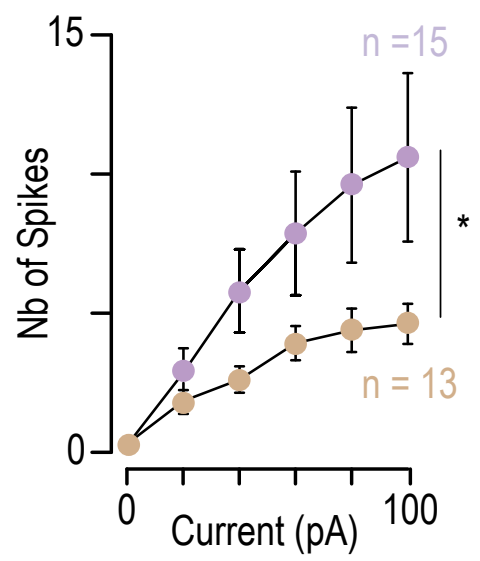

F

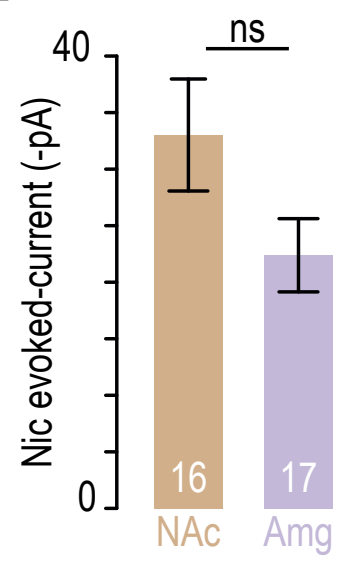

Figure S6 
Figure S7: Detailed analysis of nicotine-induced anxiety-like behaviors and the role of $\beta 2$ subunitcontaining nAChR. Related to Figure 3.

(A) Left: Individual data for Figure 3A, Sal (grey, $n=23$ ) or Nic (black, $n=21$ ), solid colors indicate group means, individual data with open gray dots (one-way RM ANOVA Nic: $F_{(2,40)}=5.18,{ }^{* *} p=0.01$; Sal: $F_{(2,44)}=$ 1.65, $p=0.2$ ). Right: Mean number of open arm entries during elevated O-maze (EOM) test (two-way RM ANOVA main time effect $\left.F_{(2,84)}=8.02,{ }^{* * *} p<0.001\right)$. (B) Locomotor activity was measured in a square open field (OF) following an intraperitoneal (IP) injection of either saline (IP Sal, grey, $n=6$ ) or nicotine (IP Nic 0.5 $\mathrm{mg} / \mathrm{kg}$, black, $\mathrm{n}=6$ ) in WT mice. No difference was observed in the distance traveled over time (two-way RM ANOVA no time, no treatment or interaction effect, $p>0.05$ ) or in the total distance traveled during 9 minutes (inset, Student's t-test, $p>0.05$ ). (C) Example of post hoc verification of intracranial guide cannula implantations in WT mice. Bilateral injection cannulas $(0.5 \mu \mathrm{m}$ longer than the guide cannulas) are inserted on the day of the experiment for local infusion into the VTA. Tyrosine hydroxylase (TH) labeling is shown in yellow. (D) Left: Individual data for Figure 3B, intracranial (IC) infusion of Sal (grey, $n=6$ ) or Nic (red, $n=7$ ) $1 \mathrm{mg} / \mathrm{mL} 1$ minute before the test (one-way RM ANOVA Nic: $F_{(2,12)}=26.11$, ${ }^{* * *} p<0.001 ;$ Sal: $F_{(2,10)}=0.01$, $p$ $=0.99$ ). Group means are shown by solid color points, individual data with open gray dots. Right: Mean number of open arm entries during EOM test (two-way RM ANOVA main time effect $F_{(2,20)}=8.25$, ${ }^{* *} p=$ 0.002 ). (E) Schematic of $\beta 2$ subunit re-expression by lentiviral vectorization in the VTA of $\beta 2^{-1-}$ mice. Lentiviruses encoding either $p$ GK- $\beta 2-$ IRES-GFP $\left(\beta 2-{ }^{-V}\right.$ ec) or pGK-GFP ( $\beta 2-1-$ GFP, used as a control) were injected into the VTA. Representative immunofluorescence image of a $\beta 2-1-\mathrm{Vec}$ mouse brain labeled for TH (red) and GFP (green). (F) Percentage of time spent in the open arms of the EOM by $\beta 2-$ GFP mice $(n=6$,

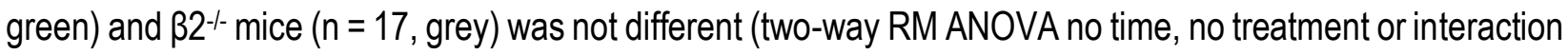
effect, $p>0.05)$. (G) Left: Individual data for Figure 3D, $\beta 2^{--}$mice $\left(n=23\right.$, green) and $\beta 2^{-1-V e c}$ mice $(n=18$, brown) after IP injection of Nic $0.5 \mathrm{mg} / \mathrm{kg} 1$ minute before the test (one-way RM ANOVA for $\beta 2^{-1-V e c}$ mice: $F_{(2,34)}=8.65,{ }^{* * *} p<0.001$; and for $\beta 2^{-1}$ mice: $\left.F_{(2,44)}=1.08, p=0.3\right)$. Group means are shown by solid color points, individual data with open gray dots. Right: Mean number of open arm entries from $\beta 2--\mathrm{GFP}$ or $\beta 2^{--}$ mice in this test (two-way RM ANOVA main time effect $F_{(2,78)}=7.26$, ${ }^{* *} p=0.001$ ).

IF: interfascicular nucleus; IPN: interpeduncular nucleus; SNc: substantia nigra pars compacta; ml: medial lemniscus; SNc: substantia nigra pars compacta. 
A $\quad \bullet N$ Nic (IP $0.5 \mathrm{mg} / \mathrm{kg}), \mathrm{n}=21$

$$
\text { - Sal, } n=23
$$

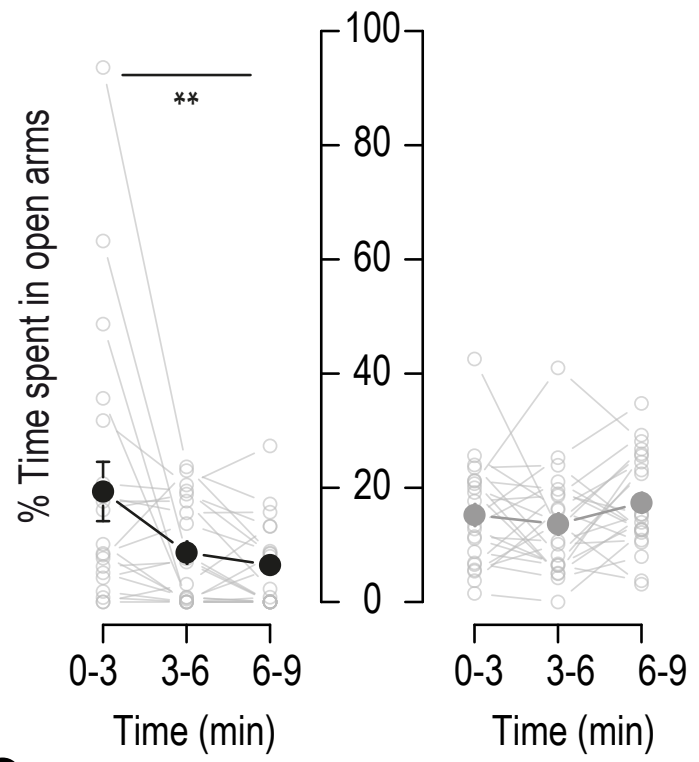

C

Implant location of bilateral guide cannulas
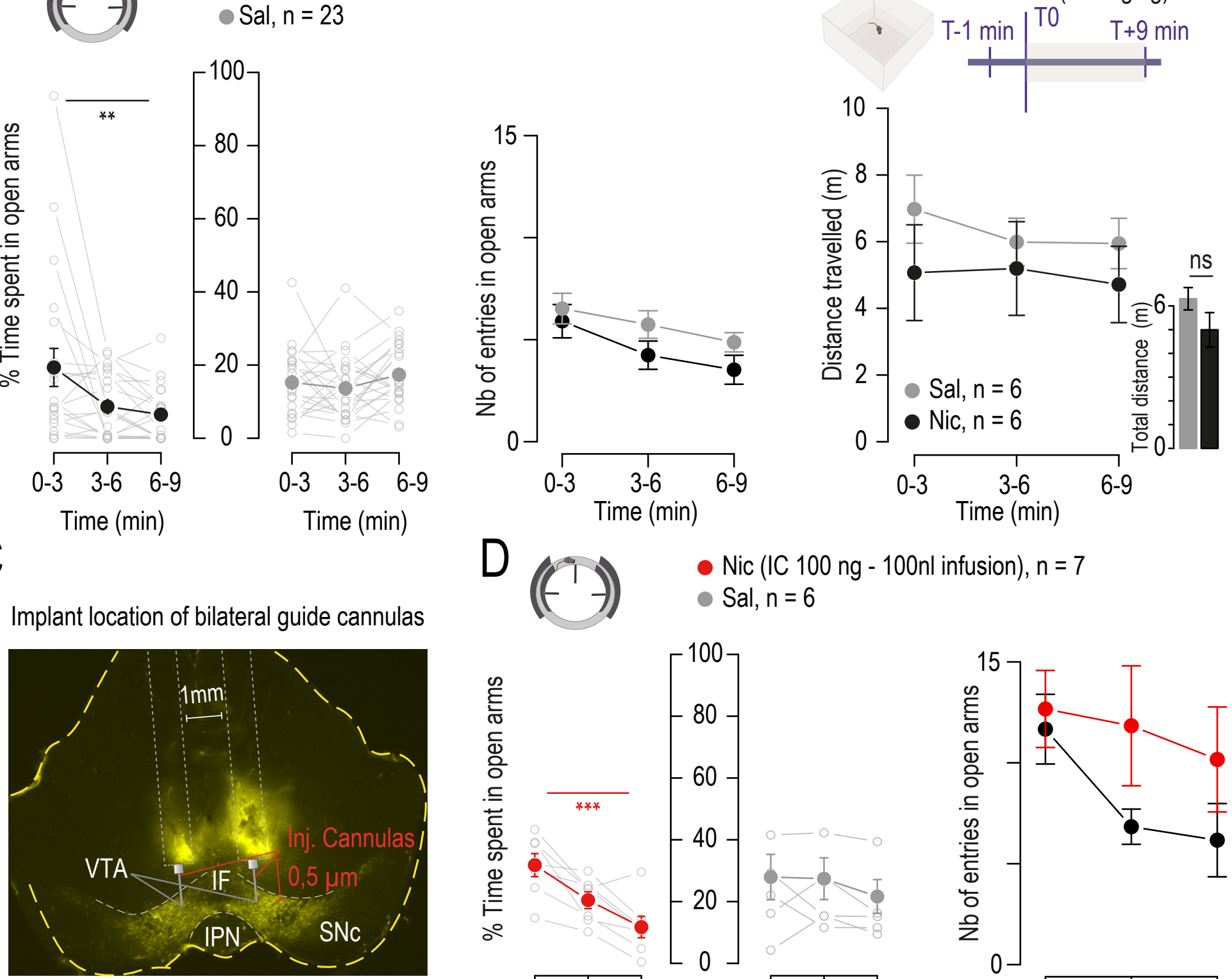

WT mice

I.P Sal

I.P Nic $(0.5 \mathrm{mg} / \mathrm{kg})$

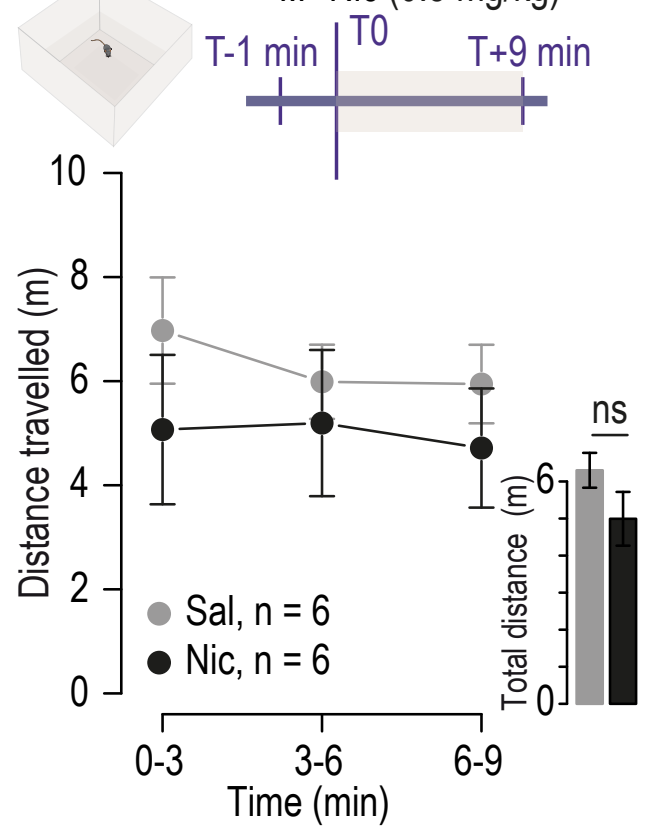

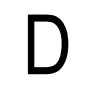

$(-7)$

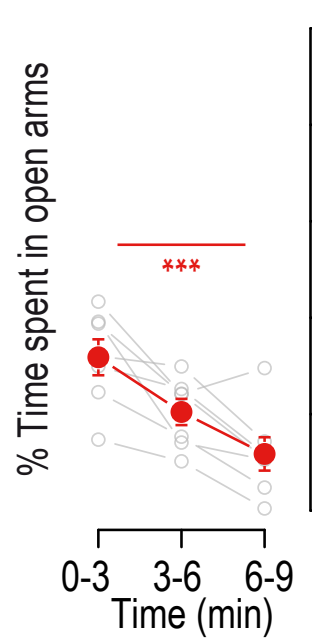

- Nic (IC $100 \mathrm{ng}$ - 100nl infusion), $\mathrm{n}=7$

- Sal, $n=6$ 
Figure S8: $\beta 2 \mathrm{nAChR}$ subunit re-expression in the VTA restores DA neuron response to nicotine injection. Related to Figure 3.

(A) Density plot of percentage of firing variation induced by IV injection of either saline (Sal, grey, $n=46$ ) or nicotine (Nic, black, $n=46$ ) in $\beta 2-$ mice (Kolmogorov-Smirnov test, $p=0.7$ ). (B) Firing rate variation $(\Delta)$ from baseline (Bas) induced by Nic and Sal injection in $\beta 2--V e c$ mice DA neurons with increase $(\Delta \mathrm{fr}>0)$ or decrease $(\Delta \mathrm{fr}<0)$ in firing frequency after Nic injection. Comparison between mean firing rate during baseline and maximum firing rate after injection (paired Wilcoxon test, ${ }^{* * *} p<0.001$ ), comparison between saline-induced and Nic-induced firing rate variation (Wilcoxon test, $p(\Delta f r>0)=0.07, p(\Delta f r<0)=0.4)$. (C) Density plot of percentage of firing variation induced by IV Sal or Nic injection in $\beta 2^{-1}-$ Vec mice (Sal: grey, $n=79$, Nic: black, $n=90$ ). (Kolmogorov-Smirnov test, ${ }^{*} p=0.024$ ). (D) Firing rate variation $(\Delta)$ from baseline (Bas) induced by $N i c$ and Sal injection in $\beta 2^{--V e c}$ mice DA neurons with increase $(\Delta f r>0)$ or decrease $(\Delta f r<0)$ in firing frequency after Nic injection. Comparison between mean firing rate during baseline and maximum firing rate after injection (paired Wilcoxon test, ${ }^{* * *} p<0.001$ ), comparison between saline-induced and Nic-induced firing rate variation (Wilcoxon test, ${ }^{* * *} p<0.001$ ). Mean scores are represented in black, and individual scores in grey. (E) Time course for the average change in firing frequency upon saline (grey) or nicotine injection for activated (Nic+, in red) and inhibited (Nic-, in blue) VTA DA neurons in $\beta 2^{--}$and $\beta 2^{--V e c}$ mice. Comparison between saline-induced and nicotine-induced firing rate variation (paired Wilcoxon test, ${ }^{* *} p=0.0015,{ }^{* * *} p<0.001$ ) (F) Firing rate variation $(\Delta)$ from baseline (Bas) induced by Nic injection in Nic+ or Nic- DA neurons in $\beta 2-$ and $\beta 2--V e c$ mice. Comparison between mean firing rate during baseline and maximum firing rate after Nic injection (paired Wilcoxon test, ${ }^{*} p=0.015,{ }^{* * *} p<0.001$ ), comparison between Nic-induced firing rate variation in $\beta 2^{--}$and $\beta 2^{--V e c}$ mice (Wilcoxon test, ${ }^{* *} p<0.001$ ). Mean scores are represented in black, and individual scores in grey. In panels $B$ and $D$, all neurons are considered, while in $E$ and $F$ only responding neurons (i.e those with a statistically significant increase ( $\mathrm{Nic}^{+}$) or decrease (Nic-) in firing rate in response to Nic injection, see bootstrapping in Methods) are included. 
A

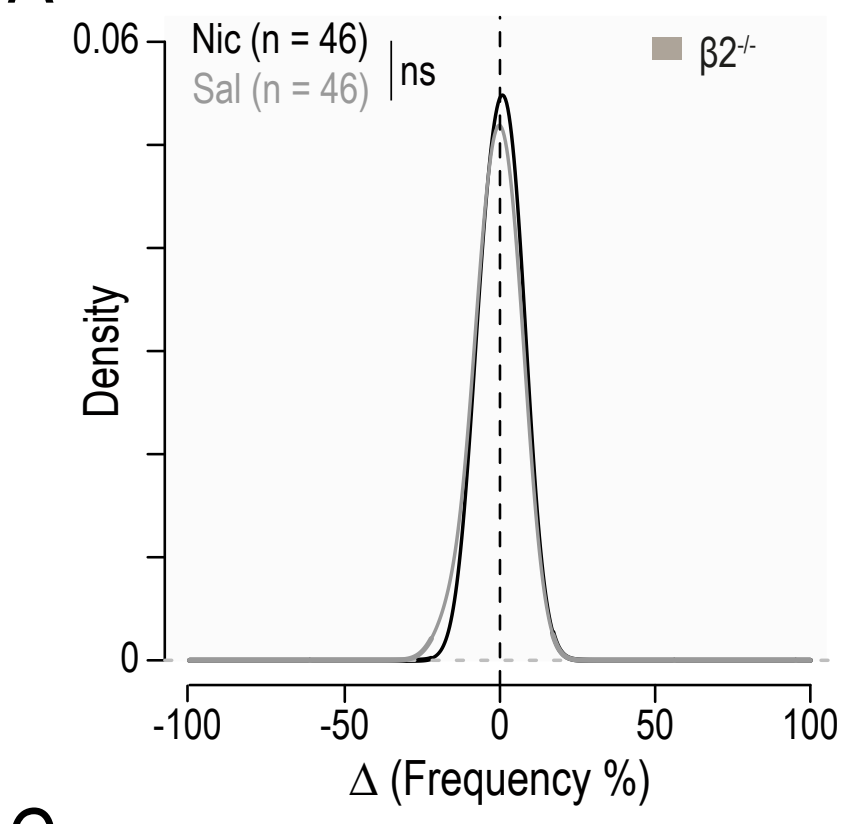

C

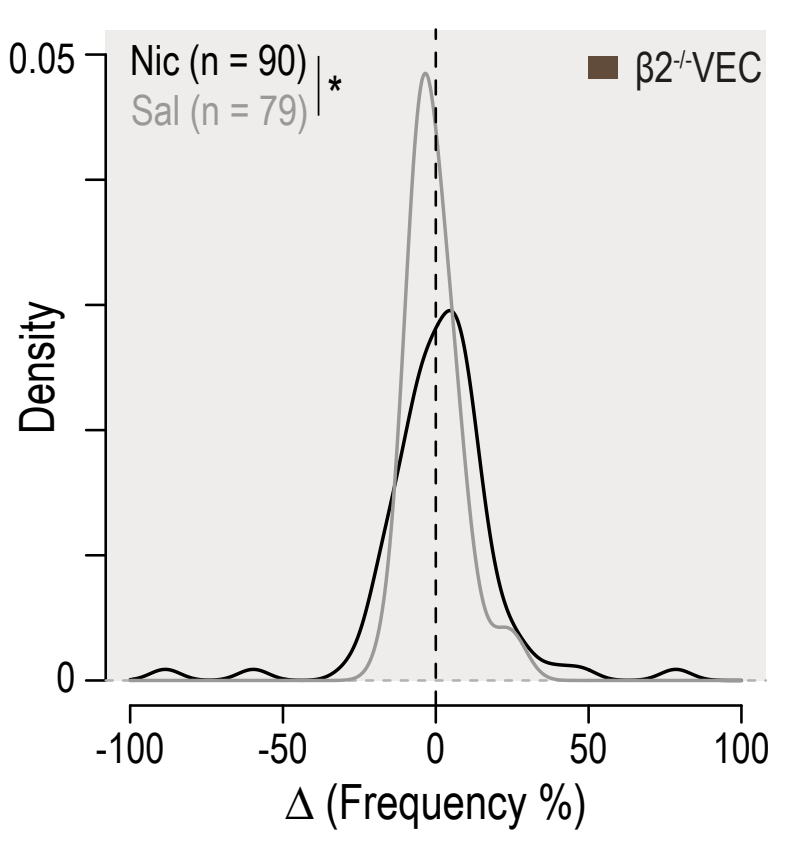

E

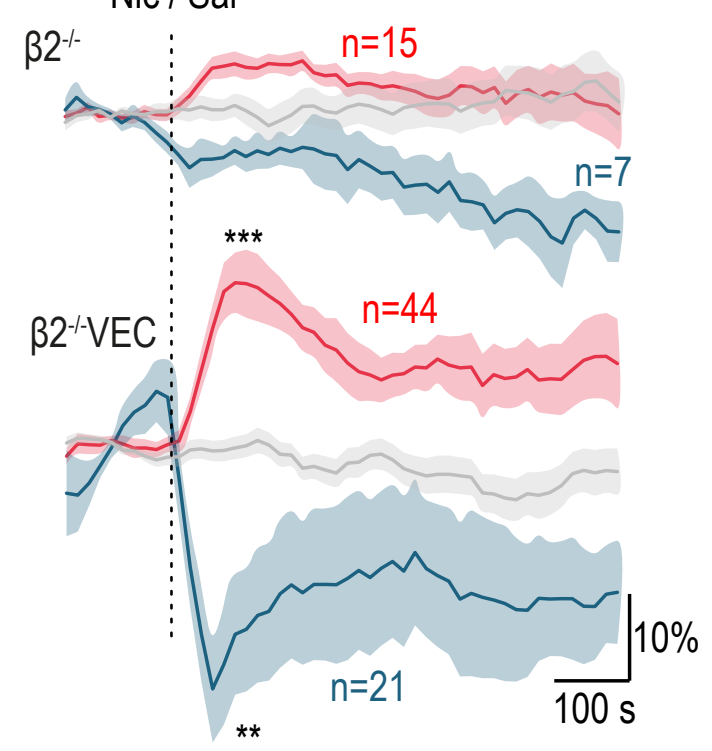

B

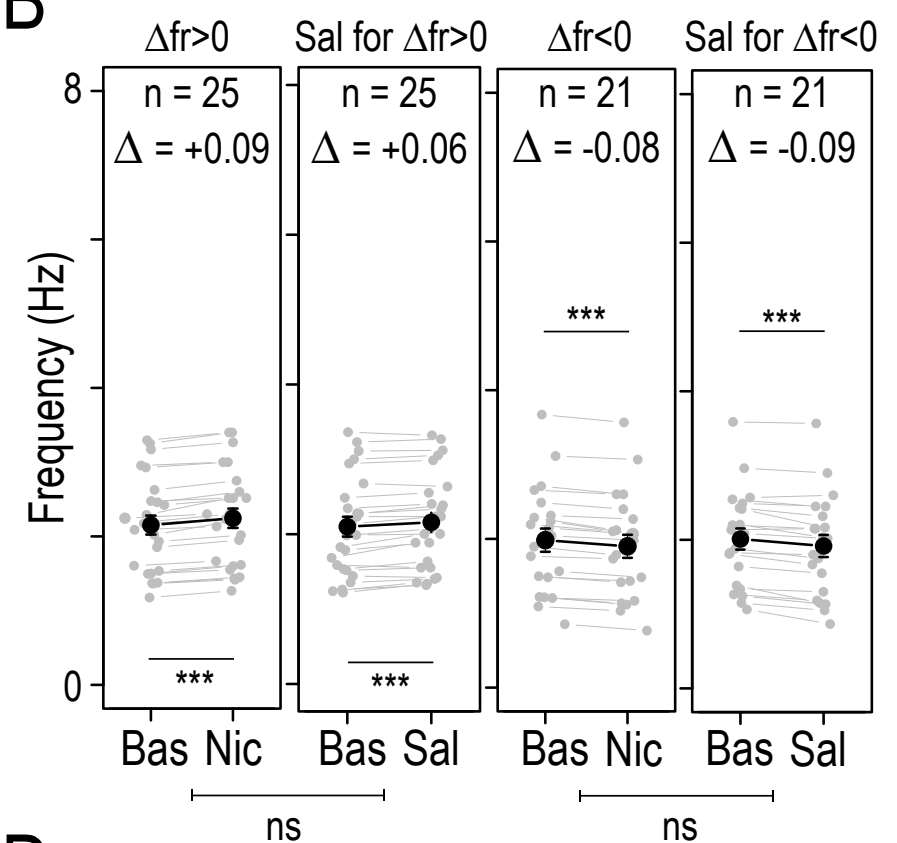

D

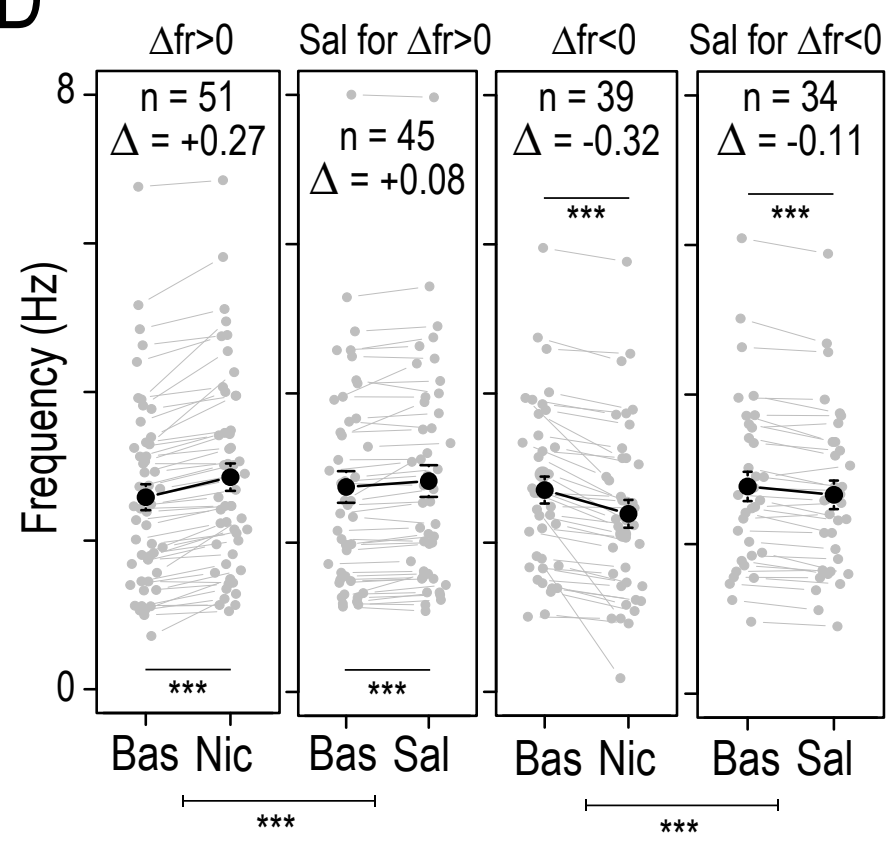

$\mathrm{F}$

$\beta 2^{--V E C N i c}+\beta 2^{--} \mathrm{Nic}+\beta 2^{--V E C ~ N i c}-\beta 2^{--} \mathrm{Nic}-$

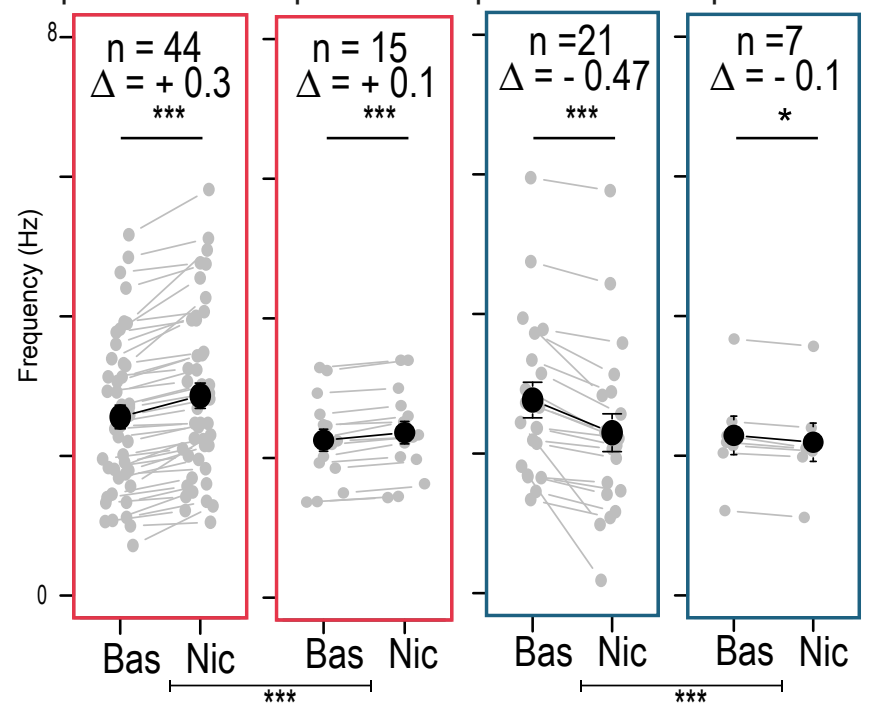


Figure S9: Temporally specific control of DA neuron firing with Jaws and CatCh. Related to Figure 4. (A) Representative immunofluorescence image of VTA DA neurons after patch-clamp recordings in mice injected with AAV-Ef1 $\alpha$-DIO-Jaws-eGFP into the VTA. Neurobiotin (NB, blue), tyrosine hydroxylase (TH, red), GFP (green) (scale bar $20 \mu \mathrm{m}$ ). (B) Example of a recording trace of a VTA DA neuron during continuous light stimulation (highlighted in yellow, $20 \mathrm{~s}, 520 \mathrm{~nm}$ ) and raster plot of action potentials showing light-induced inhibition in Jaws-expressing DA neurons $(n=7)$. (C) Representative immunofluorescence image of VTA DA neurons (NB, blue; TH, red; GFP, green) after patch-clamp recording in mice injected with AAV-DIO-hCatChYFP into the VTA (scale bar $20 \mu \mathrm{m}$ ). (D) Example of recording trace of a DA neuron of the VTA during light stimulation (10 Hz, 5-ms pulse, $470 \mathrm{~nm}$ ) and light-evoked inward current in DA neurons expressing CatCh. Mean light-evoked currents in seven DA neurons. 


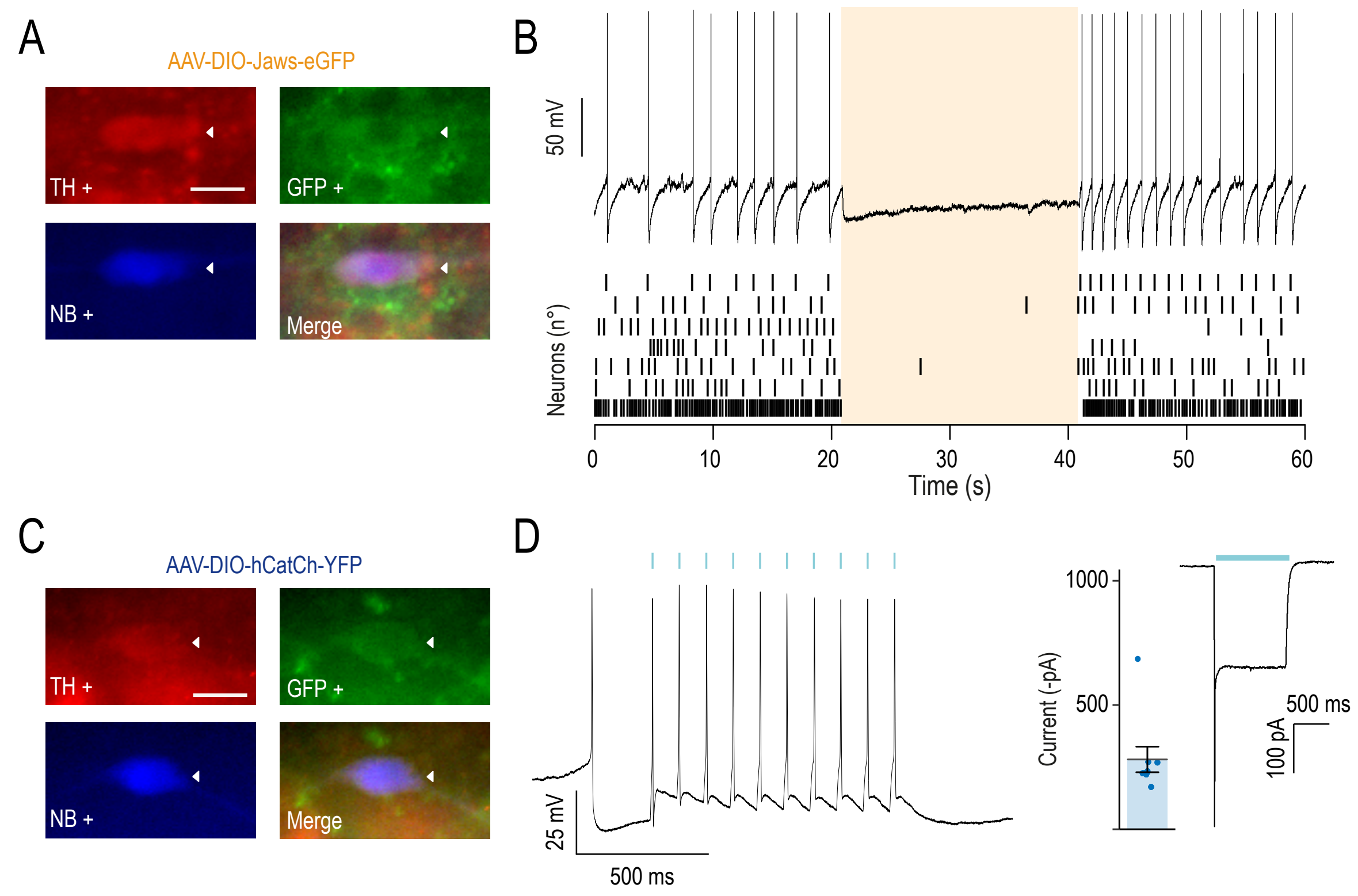

Figure S9 
Figure S10: Fiber locations for optogenetic experiments. Related to Figure 4.

(A) Representative immunofluorescence images of VTA sections after AAV-DIO-Jaws-eGFP or AAV-DIOYFP injection into the VTA. (B) Representative immunofluorescence images of VTA sections after AAV-Ef1 $\alpha$ DIO-hCatCh-YFP or AAV-Ef1 $\alpha$-DIO-YFP injection into the VTA. (C) Verification of fiber implantations into the basolateral Amg (BLA) of mice used in optogenetic experiments, positioned onto Paxinos atlas slices from bregma -1.22 to $-1.82 \mathrm{~mm}$. Left side: orange dots indicate fiber tip location for mice injected with Jaws $(n=$ 13), and green dots indicate fiber location in YFP controls $(n=10)$. Right side: blue dots indicate fiber tip location for mice injected with CatCh $(n=16)$, and green dots indicate fiber location in YFP controls $(n=11)$. (D) Verification of fiber implantations into the NAc lateral shell (NAcLSh) of mice used in optogenetic experiments, positioned onto Paxinos atlas slices from bregma +0.86 to $+1.54 \mathrm{~mm}$. Left side: blue dots indicate fiber tip location for mice injected with CatCh $(n=13)$, and green dots indicate fiber tip positions in YFP controls $(n=14)$. Right side: orange dots indicate fiber tip location for mice injected with Jaws $(n=11)$, and green dots indicate fiber tip location in YFP controls $(n=12)$. 
A

B

AAV-DIO-Jaws-eGFP
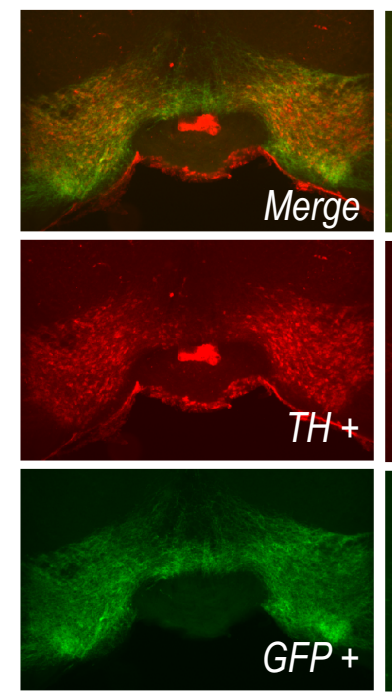

C
AAV-DIO-YFP
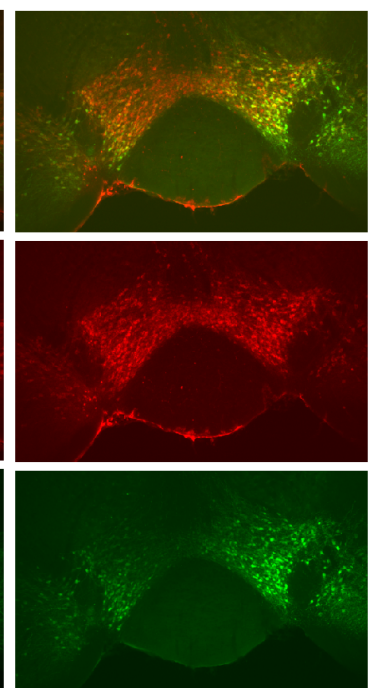

AAV-DIO-Jaws-eGFP

Optical AAV-DIO-hCatCh-YFP fibers AAV-DIO-YFP

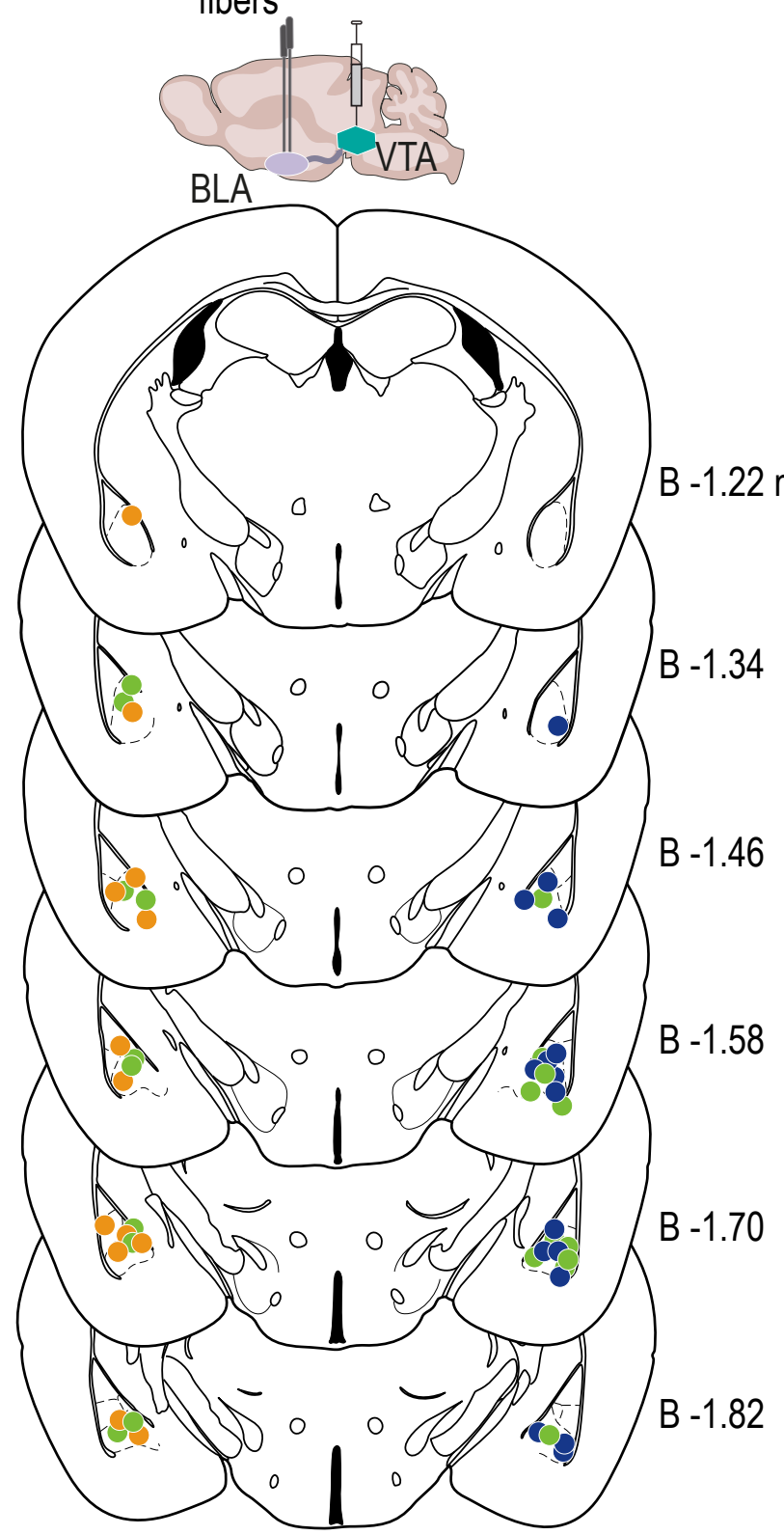

AAV-DIO-hCatCh-YFP
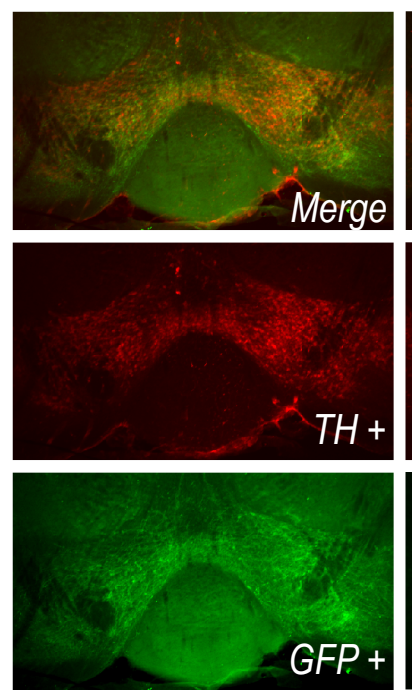

D
AAV-DIO-YFP
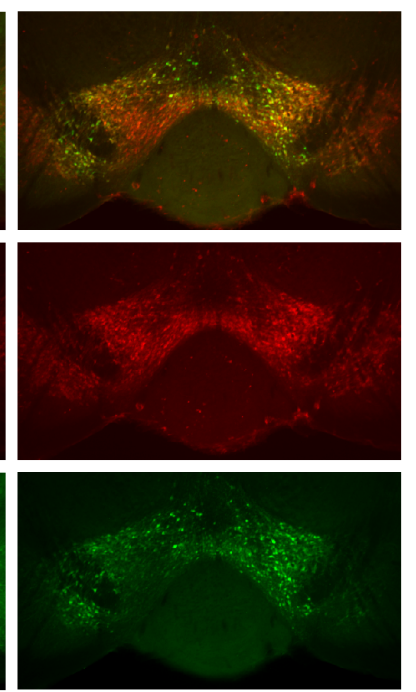

AAV-DIO-Jaws-eGFP Optical AAV-DIO-hCatCh-YFP fibers AAV-DIO-YFP

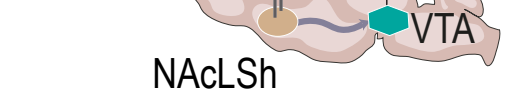

Figure S10 
Figure S11: Detailed analysis of optogenetic effects on elevated O-maze test and locomotor behavior. Related to Figure 4.

(A) Left: Individual data for Figure $4 A$ (Jaws, $n=18$, orange, one-way RM ANOVA $F_{(2,34)}=5.28,{ }^{* *} p=0.01$; YFP, $n=19$, green, one-way RM ANOVA $\left.F_{(2,36)}=0.32, p=0.7\right)$. Middle: Time spent in open arms for mice injected with Jaws during the 10 first minute of EOM, divided in two groups depending on the location of the animal, either in open arms (OA, white) or closed arms (CA, gray) at the onset of the stimulation (two-way RM ANOVA OA vs CA main light effect $F_{(1,17)}=9.02,{ }^{* *} p=0.008$, no effect of position $F_{(1,17)}=0.003, p=$ 0.96). Right: Open arm entries during EOM (two-way RM ANOVA Jaws vs YFP no effect of time or opsin). Group means are shown by solid color points, individual data with open gray dots. (B) Left: Individual data for Figure 4B (CatCh, $n=18$, blue, one-way RM ANOVA $F_{(2,34)}=9.27$, *** $p<0.001$; YFP, $n=19$, green, oneway ANOVA $\left.F_{(2,36)}=0.01, p=0.99\right)$. Middle: Time spent in open arms for mice injected with CatCh during the 10 first minutes of EOM, divided in two groups depending on the location of the animal, either in open arms (OA, white) or closed arms (CA, gray) at the onset of the stimulation (two-way RM ANOVA OA vs CA main light effect $F_{(1,16)}=16.56,{ }^{* * *} p<0.001$, no effect of position $\left.F_{(1,16)}=0.32, p=0.57\right)$. Right: Open arm entries during EOM (two-way RM ANOVA CatCh vs YFP no effect of time or opsin). Group means are shown by solid color points, individual data with open gray dots. (C) Locomotor activity was assessed in a novel open field (OF) in the three paired groups. Top: Jaws-injected $(n=20)$ and YFP-injected mice $(n=20)$ with fibers implanted in the BLA (two-way RM ANOVA, main time effect $F_{(2,76)}=44.27$, ${ }^{* * *} p<0.001$, no opsin or interaction effect). Bottom: CatCh-injected $(n=13)$ and YFP-injected mice $(n=10)$ implanted in the BLA (twoway RM ANOVA, main time effect $F_{(2,42)}=25.17,{ }^{* * *} p<0.001$, no opsin or interaction effect). 
A Optical AAV-DIO-hCatCh-YFP
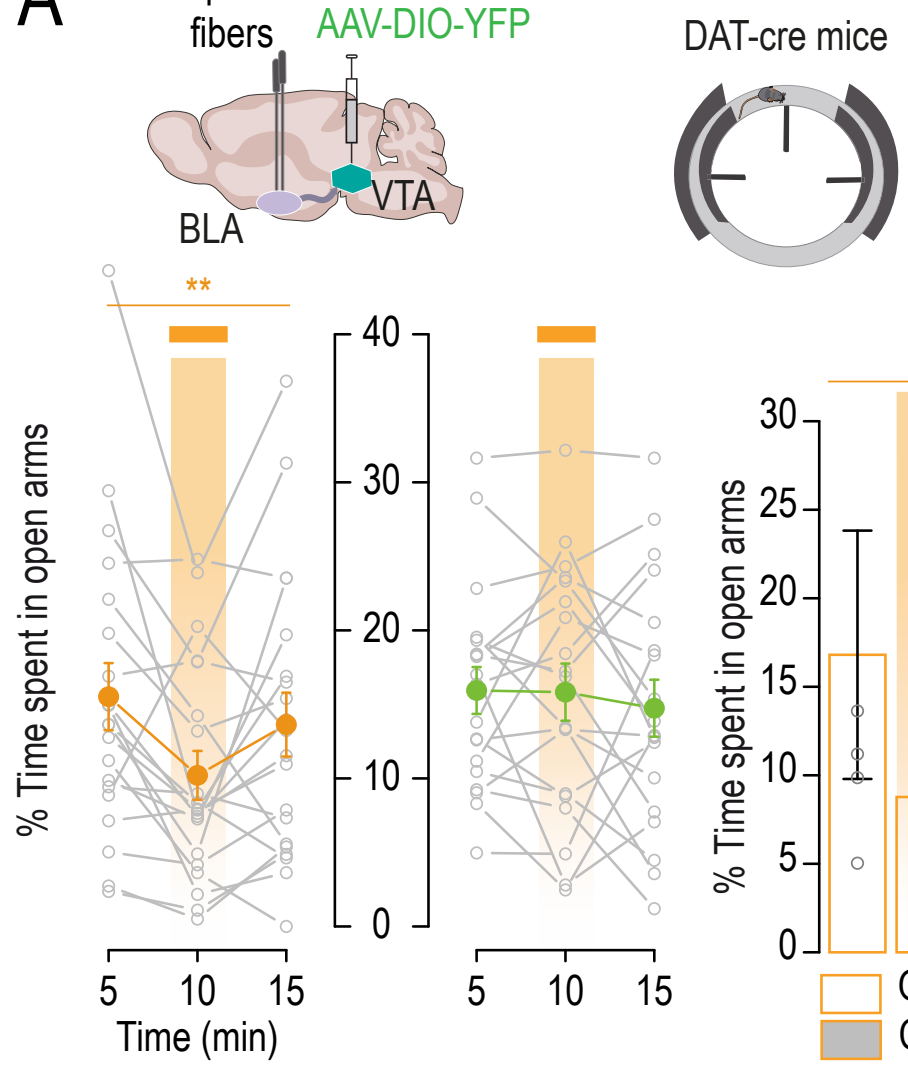

B

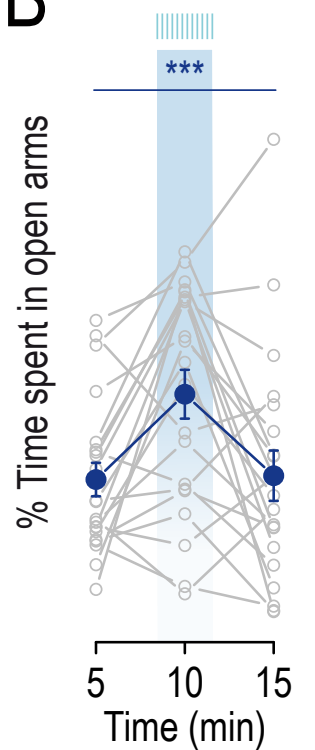

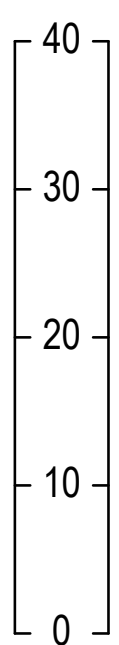
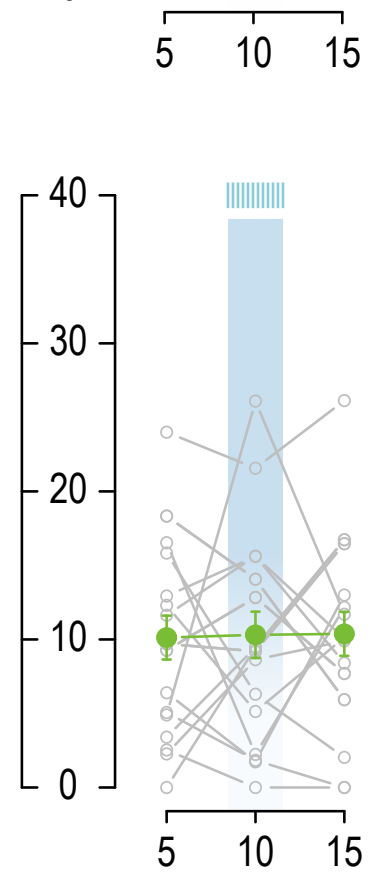
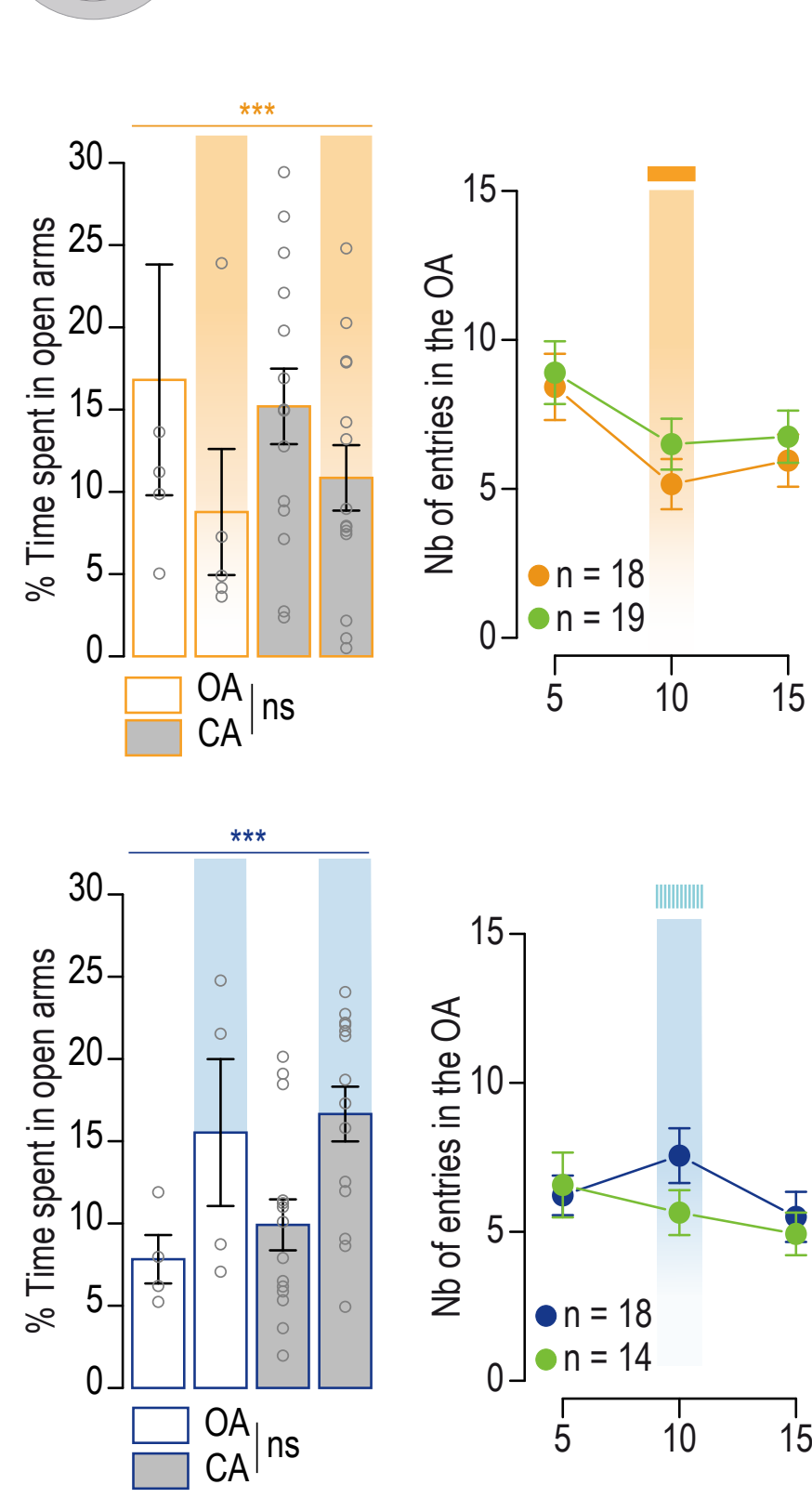

C
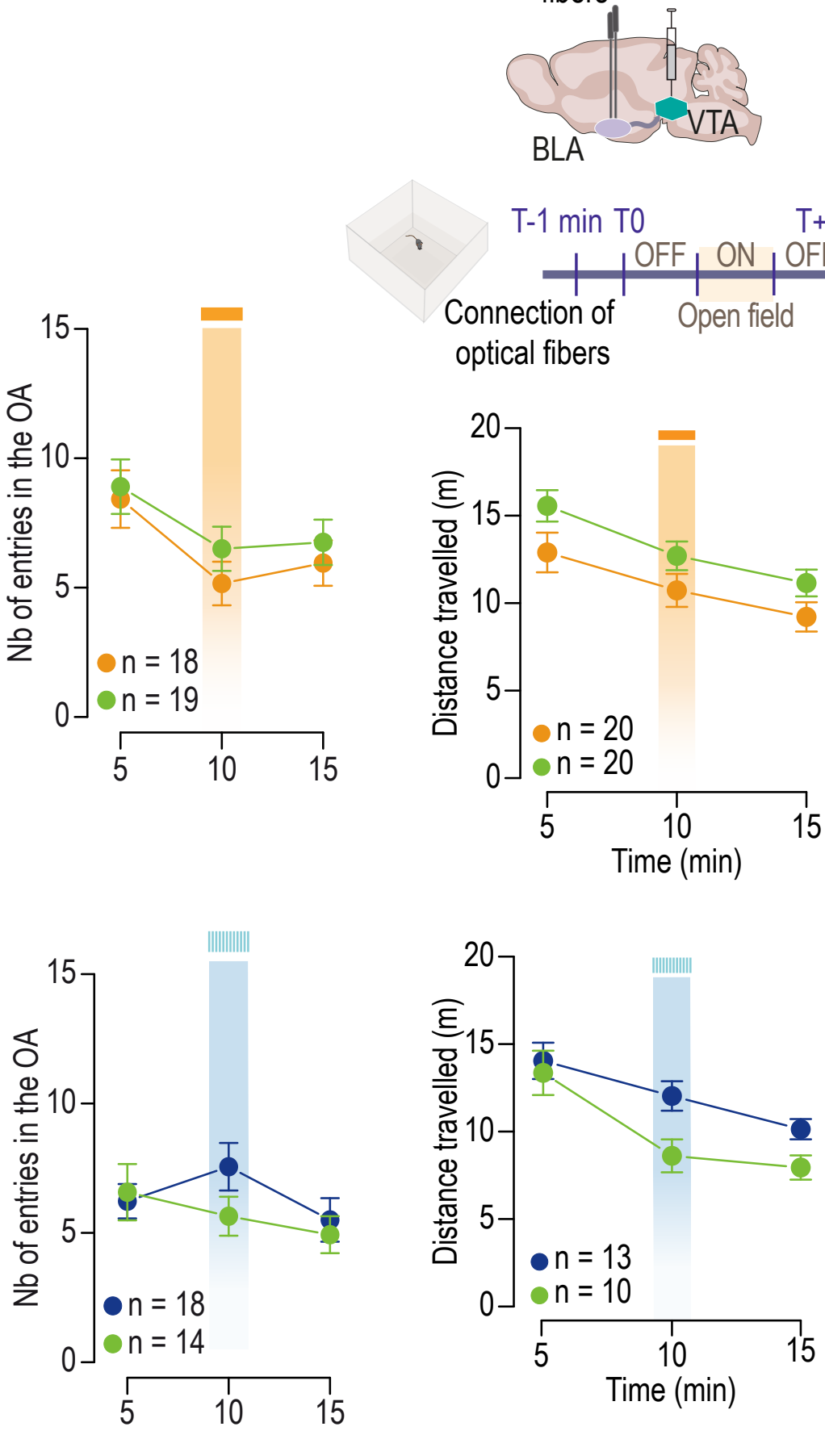

Figure S11 
Figure S12: Inhibition of VTA terminals in the BLA, but not the CeA, produces anxiety-like behavior. Related to Figure 4.

(A) Left: Representative immunofluorescence image of a VTA section with AAV-CAG-Jaws-GFP (Jaws) expression (GFP: green labeling, TH: red labeling). Right. Optical fibers were implanted in the basolateral Amg (BLA) of wild-type (WT) mice injected either with Jaws (orange dots represent the fiber tip location, $n=$ 7) or with AAV-CAG-GFP (GFP, green dots, $n=3$ ) in the VTA. (B) Left: Representative immunofluorescence image of a VTA section with Jaws expression (GFP: green labeling, TH: red labeling). Right: Optical fibers were bilaterally implanted in the central amygdala $(\mathrm{CeA})$ of a separate group of WT mice injected with either Jaws (orange dots represent the fiber tip location, $n=7$ ) or with GFP (green dots, $n=7$ ) in the VTA. (C) Photo-inhibiting VTA axon terminals in the BLA using Jaws during the EOM in the same mice induced a decrease in the time spent in the open arms compared to the control group (two- way RM ANOVA, time $x$ opsin interaction $F_{(2,42)}=3.44,{ }^{*} p=0.04$, post hoc Student's t-test $p$ (ON Jaws vs GFP) $=0.056$; post hoc Student's t-test with Bonferroni corrections Jaws ${ }^{*} p$ (5 minutes vs 10 minutes) $=0.01 ;{ }^{* *} p$ (10 minutes vs 15 minutes $)=0.005$ ). (D) Photo-inhibiting VTA terminals in the CeA did not produce any difference in the amount of time spent in the open arm of the EOM test (two-way RM ANOVA, time $x$ opsin interaction $F_{(2,32)}=3.67$, * $p=0.04$, post hoc Student's t-test with Bonferroni corrections $p>0.05$ ). (E) Mice implanted in the BLA were tested for any difference in locomotor activity between groups in the open field (OF). The test lasted 15 minutes and consisted of a 5 -minute period of photostimulation (continuous at $520 \mathrm{~nm}$ ) in between two nonstimulation periods (OFF-ON-OFF). During both OFF- and ON-periods, the groups did not present any statistically significant difference in the distance traveled in the OF (two-way RM ANOVA main time effect $F_{(2,44)}=5.89,{ }^{* *} p=0.005$, no opsin or interaction effect, post hoc Student's t-test with Bonferroni corrections, $p>0.05)$. (F) Photo-inhibiting VTA terminals in the CeA did not produce any statistically significant difference in locomotor activity in the OF test between the Jaws- and GFP-expressing groups (two-way RM ANOVA main time effect $F_{(2,32)}=23.11$, ${ }^{* * *} p<0.001$, time $x$ opsin interaction $F_{(2,32)}=3.8,{ }^{*} p=0.03$, no opsin effect, post hoc Student's t-test with Bonferroni corrections, $p>0.05$ ).

IF: interfascicular nucleus; IPN: interpeduncular nucleus; ml: medial lemniscus; SNc: substantia nigra pars compacta. 
Figure S13: Detailed analysis of optogenetic stimulations of NAcLSh in elevated 0-maze test and locomotor behavior. Related to Figure 5.

(A) Left: Individual data for Figure $5 A$ (CatCh, $n=13$, blue, one-way RM ANOVA $F_{(2,24)}=0.61, p=0.6$; YFP, $n=14$, green, one-way RM ANOVA $\left.F_{(2,26)}=1.47, p=0.3\right)$. Middle: Time spent in open arms for mice injected with CatCh during the 10 first minute of EOM, divided in two groups depending on the location of the animal, either in open arms (OA, white) or closed arms (CA, gray) at the onset of the stimulation (two-way RM ANOVA $O A$ vs $C A$ no effect of light $F_{(1,11)}=0.46, p=0.5$, or position $\left.F_{(1,11)}=1.35, p=0.27\right)$. Right: Open arm entries during EOM (two-way RM ANOVA CatCh vs YFP no effect of time or opsin). Group means are shown by solid color points, individual data with open gray dots. (B) Left: Individual data for Figure 5B (Jaws, $n=12$, orange, one-way RM ANOVA $F_{(2,22)}=0.25, p=0.8 ; Y F P, n=12$, green, one-way RM ANOVA $\left.F_{(2,22)}=0.93, p=0.4\right)$. Middle: Time spent in open arms for mice injected with Jaws during the 10 first minute of EOM, divided in two groups depending on the location of the animal, either in open arms (OA, white) or closed arms (CA, gray) at the onset of the stimulation (two-way RM ANOVA OA vs CA no effect of light $F_{(1,11)}=0.18, p=0.67$, or position $F_{(1,11)}=1.9, p=0.19$ ). Right: Open arm entries during EOM (two-way RM ANOVA Jaws vs YFP no effect of time or opsin). Group means are shown by solid color points, individual data with open gray dots. (C) Locomotor activity was assessed in a novel open field (OF) in the three paired groups. Top: CatCh-injected $(n=13)$ and YFP-injected mice $(n=14)$ implanted in the NAcLSh (two-way RM ANOVA, main time effect $F_{(2,50)}=14.27,{ }^{* * *} p<0.001$, time $x$ opsin interaction $F_{(2,50)}=4,{ }^{*} p=0.02$, post hoc Wilcoxon test for CatCh vs YFP at 5 minutes, $\left.{ }^{*} p=0.04\right)$. Bottom: Jaws-injected $(n=12)$ and YFP-injected mice $(n=12)$ implanted in the NAcLSh (two-way RM ANOVA, main time effect $F_{(2,44)}=12.47$, ${ }^{* *} p<0.001$ ). 

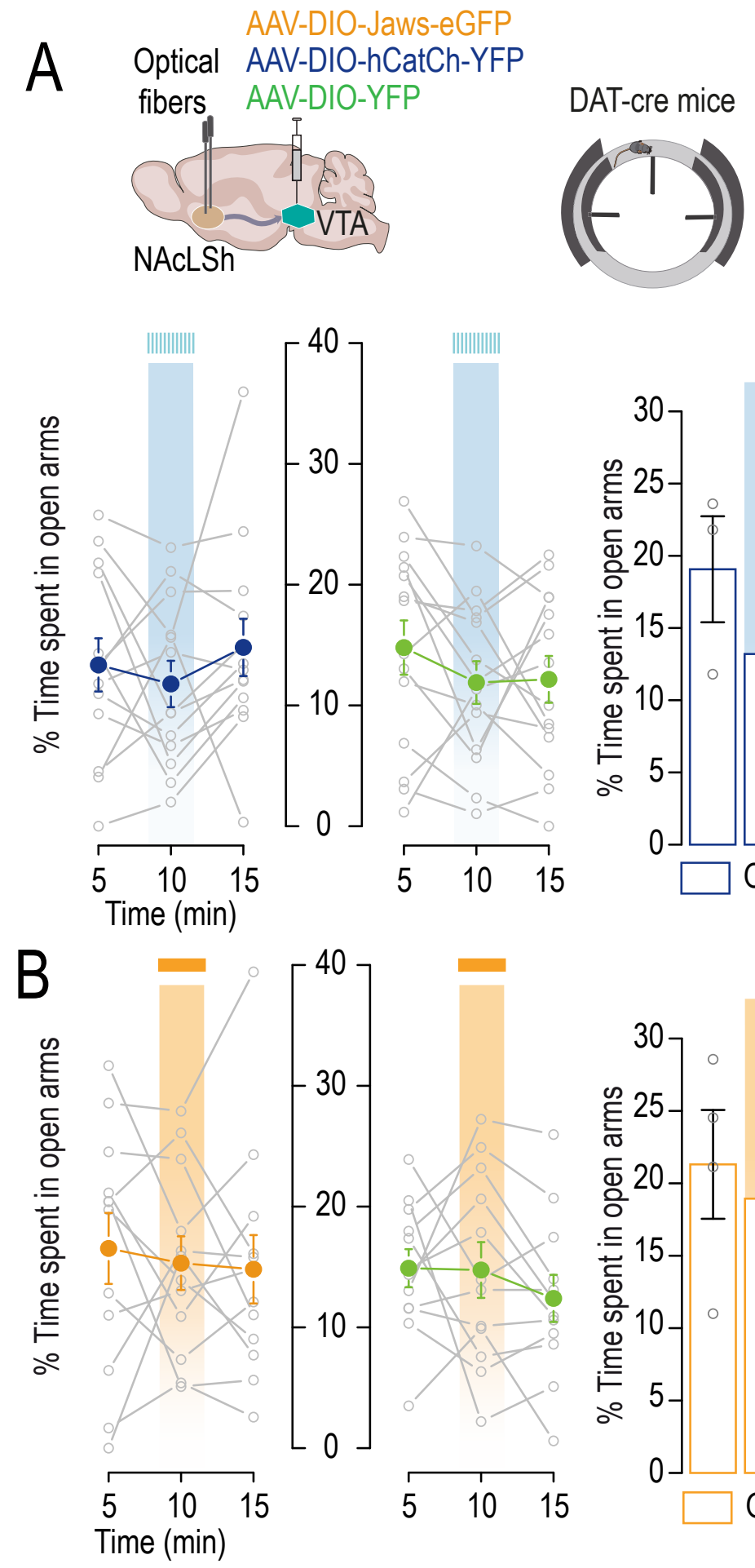

C
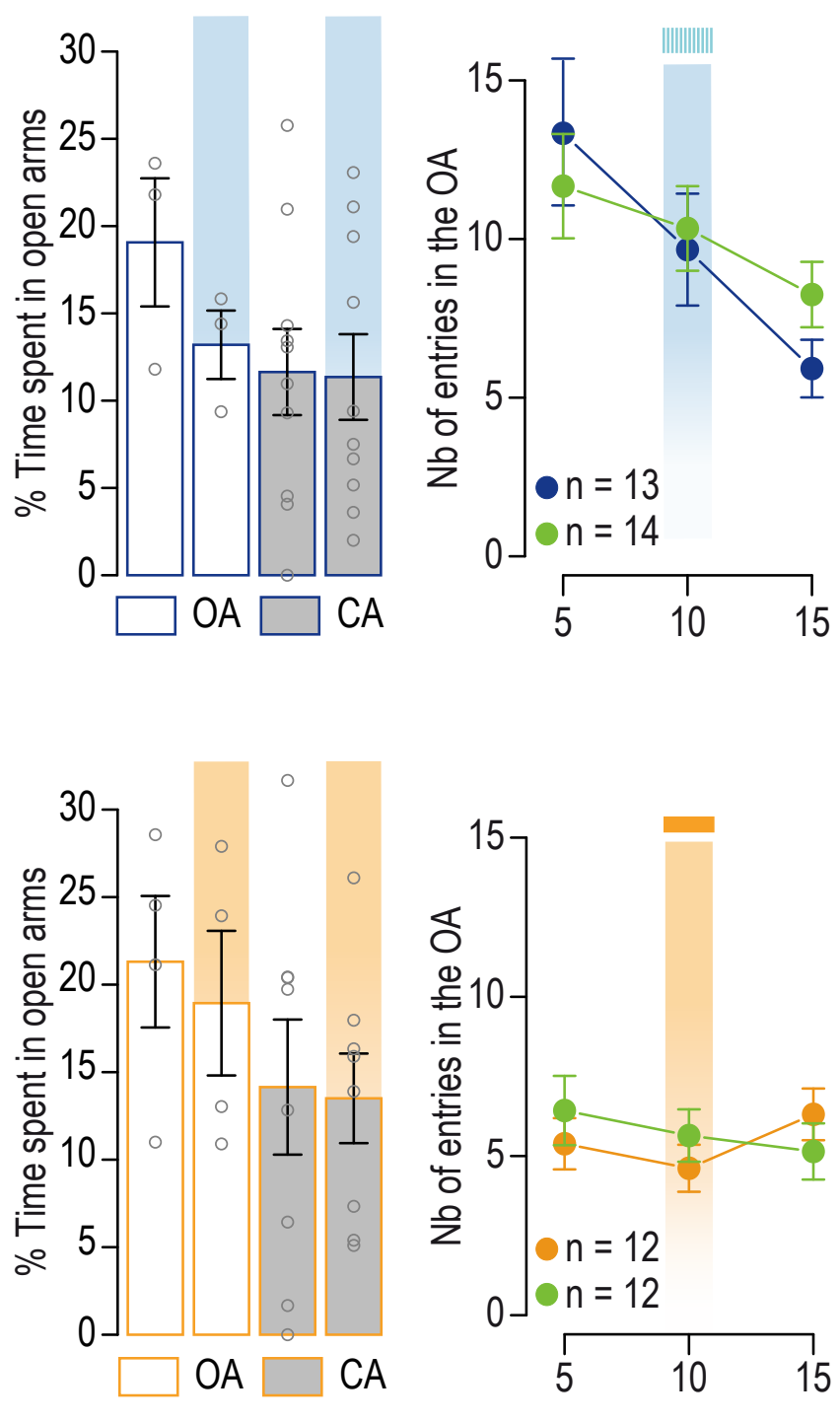

AAV-DIO-Jaws-eGFP

Optical AAV-DIO-hCatCh-YFP

fibers AAV-DIO-YFP
$\mathrm{T}+15 \mathrm{~min}$
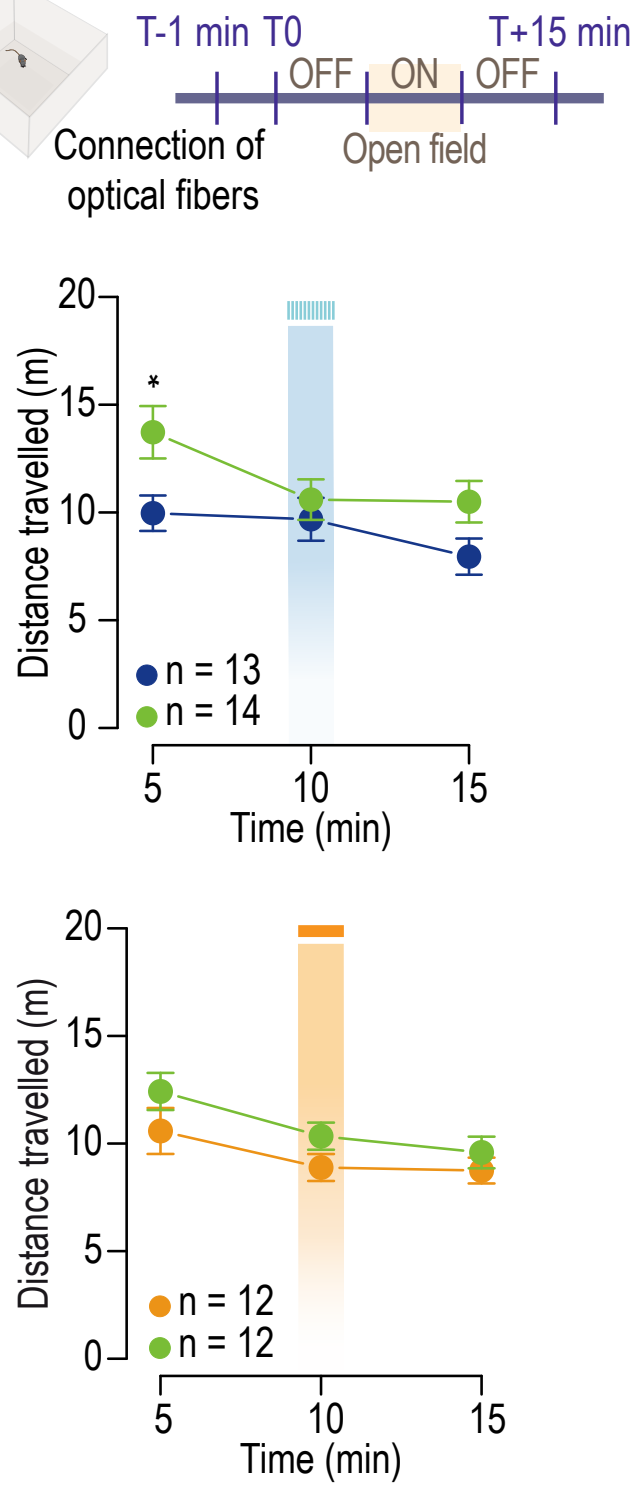

Figure S13 
Figure S14: Activation of VTA terminals in the NAcMSh changes the EOM exploration and increases locomotor activity but not place preference. Related to Figure 5 .

(A) Top: Representative immunofluorescence image of a VTA section with AAV-hSyn-Jaws-GFP (Jaws) expression (GFP: green labeling, TH: red labeling). Bottom: Optical fibers were bilaterally implanted in the NAc medial shell (NAcMSh: bregma 1.7; lateral 1.75; ventral $4.25 \mathrm{~mm}$, angle $12^{\circ}$ ) of wild-type (WT) mice injected into the VTA with either AAV-hSyn-ChR2-eYFP (ChR2, blue dots represent fiber tip locations, $n=8$ ), Jaws (orange dots, $n=7$ ) or AAV-hSyn-eGFP as a control (GFP, green dots, $n=10$ ) into the VTA. (B) Top: Activating VTA axon terminals in the NAcMSh by ChR2 photostimulation during the EOM task induced an increase in both the number of entries into the open arm (two-way RM ANOVA main time effect $F_{(2,28)}=15.68$, ${ }^{* * *} p<0.001$, time $x$ opsin interaction $F_{(2,28)}=7.59,{ }^{* *} p=0.002$; post hoc Student's t-test for ChR2 vs GFP mice: ${ }^{* *} p(\mathrm{ON})=0.007$; post hoc Student's t-test with Bonferroni corrections for ChR2 mice, ${ }^{* * *} p$ (5 vs 10 minutes) $<0.001,{ }^{* * *} p$ (10 vs 15 minutes) $=0.001$ ) and in the percentage of time spent by the mice in the open arms compared to the control group (two-way RM ANOVA main time effect $F_{(2,28)}=7.28$, ${ }^{* *} p=0.003$, opsin $F_{(1,14)}=5.26,{ }^{*} p=0.038$, time $x$ opsin interaction $F_{(2,28)}=4.53,{ }^{*} p=0.02$; post hoc Student's t-test for ChR2 vs GFP mice: ${ }^{* * *} p(\mathrm{ON})<0.001$; post hoc Student's t-test and Wilcoxon test with Bonferroni corrections for ChR2 mice, $p$ (5 vs 10 minutes) $=0.06,{ }^{* *} p$ (10 vs 15 minutes) < 0.001). Bottom: Inhibiting VTA axon terminals in the NAcMSh by Jaws photostimulation during the EOM task did not alter the number of entries into the open arm, nor the percentage of time mice spent in the open arms (two-way RM ANOVA no time or opsin effect, nor interaction effect). (C) Top: Locomotor activity assessed in ChR2- and GFP-expressing groups in a square novel open field (OF). The OF test lasts 15 minutes, with $10 \mathrm{~Hz}$ photostimulation at 470 $\mathrm{nm}, 5$-ms pulses, during a 5-minute ON period in between two non-stimulation periods (OFF-ON-OFF). Activating VTA terminals in the NAcMSh by ChR2 photostimulation produced a significant increase of locomotor activity compared to GFP-expressing mice (two-way RM ANOVA main time effect $\mathrm{F}_{(2,28)}=38.45$, ${ }^{* * *} p<0.001$, opsin $F_{(1,14)}=17.93,{ }^{* * *} p<0.001$, time $x$ opsin interaction $F_{(2,28)}=18.45,{ }^{* * *} p<0.001$; post hoc Student's t-test with Bonferroni corrections for ChR2 mice, ${ }^{* * *} p$ (5 vs 10 minutes) $<0.001,{ }^{* * *} p$ (10 vs 15 minutes) $<0.001)$. Bottom: Locomotor activity assessed in Jaws- and GFP-expressing groups, where the stimulation occurs continuously at $520 \mathrm{~nm}$ over the 5 -minute ON period. Inhibiting VTA terminals in the NAcMSh by Jaws photostimulation did not alter locomotor activity in comparison to GFP-expressing mice (two-way RM ANOVA no time or opsin effect nor interaction effect). (D) The number of entries in the open arms (two-way RM ANOVA main time effect $F_{(2,20)}=5.27,{ }^{*} p=0.014$ ) and the percentage of time spent in the open arms of the EOM after nicotine IP injection in Jaws or GFP injected mice stimulated continuously throughout the test in the NAcMSh (two-way RM ANOVA main time effect $F_{(2,20)}=7.88$, ${ }^{* *} p=0.003$, and effect of opsin $\left.F_{(1,10)}=7.79,{ }^{*} p=0.019\right)$. (E) Preference score in 20min-RTPP defined by the $\%$ of time spent in the compartment where the animals are photo-stimulated compared to the compartment where they are not (ON-OFF). Neither activation (Student t-test, $p=0.4$ ) nor inhibition (Student t-test, $p=0.9$ ) of the DA axon terminals in the NAcMSh induced place preference or aversion.

IF: interfascicular nucleus; IPN: interpeduncular nucleus; ml: medial lemniscus; SNc: substantia nigra pars compacta; VTA: ventral tegmental area 
